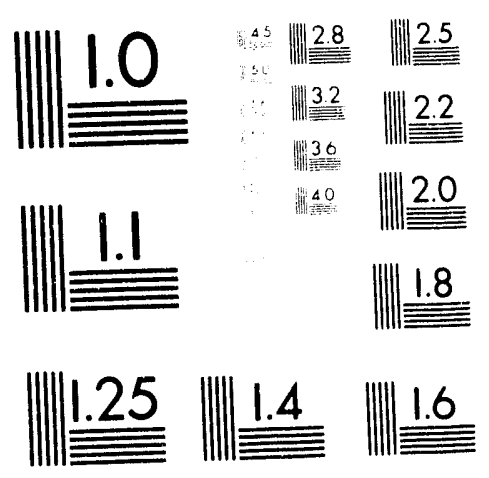



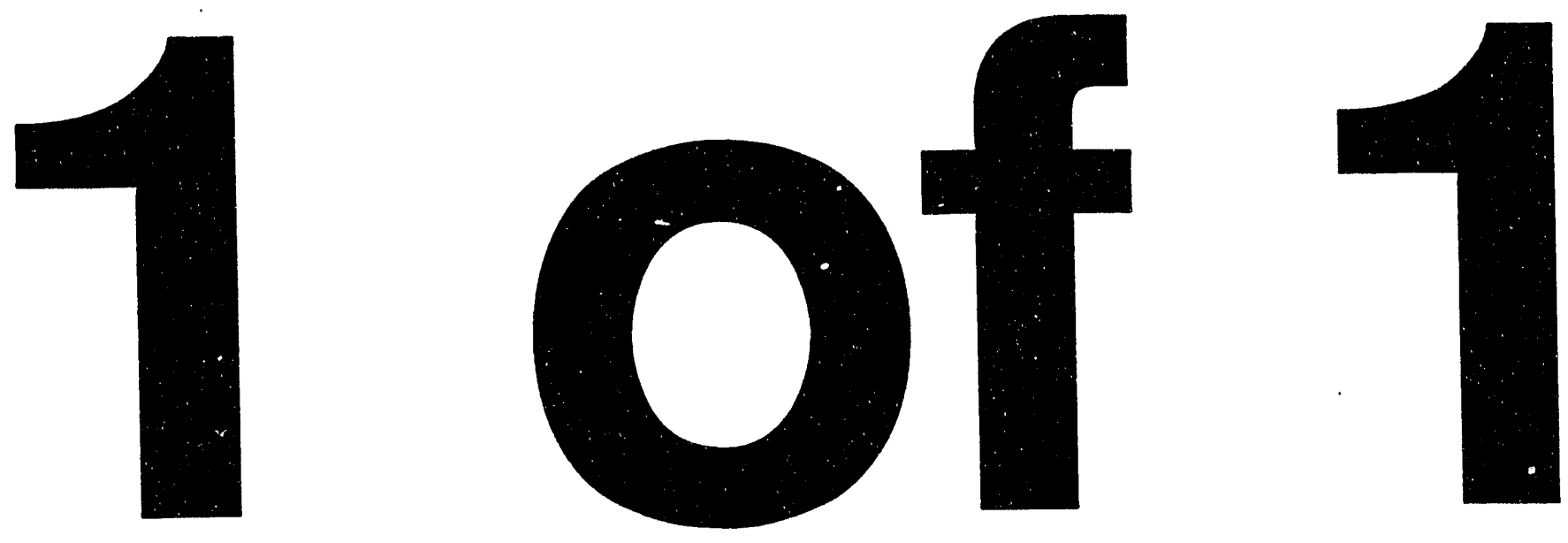


\section{Chemical Analysis}

of Selected Pothole Water Sources

in Southwestern National Parks,

Monuments, and Recreation Areas

Ernest S. Gladney

Tim Graham*

Roger W. Ferenbaugh

Michael G. Bell

Colleen Burns

Janet D. Morgan

Eric J. Nickell ${ }^{* *}$ 


\title{
CHEMICAL ANALYSIS OF SELECTED POTHOLE WATER SOURCES IN SOUTHWESTERN NATIONAL PARKS, MONUMENTS, AND RECREATION AREAS
}

\author{
by
}

\author{
Ernest S. Gladney, Tim Graham, Roger W. Ferenbaugh, Michael G. Bell, Colleen Burns, \\ Janet D. Morgan, and Eric J. Nickell
}

\begin{abstract}
Environmental data related to the evaluation of inorganic air pollution input to pothole ecosystems in the desert Southwest have been collected over the past several years. Chemical composition of pothole waters and associated sediments are reported. The enrichment factor approach has been applied to the sediments in an effort to identify elemental levels that diverge from mean crustal abundances. These data provide a baseline for determining changes in elemental concentration and enrichment status in the future.
\end{abstract}

\section{INTRODUCTION}

Acid deposition in the arid Southwest has only recently been recognized as a problem. Because precipitation events are scarce and infrequent in deserts, pollutants were thought to be adequately diluted and dispersed before they were carried to the Earth's surface in rain or snow. Alkaline soils have high buffering capacity, and most surface and ground waters are also alkaline ${ }^{1}$, which should also reduce risk from acidic deposition. Sources of acidic pollutants are not as concentrated as in the eastern United States.

These broad generalizations do not hold for all ecosystems in arid and semi-arid parts of the Southwest. Acidic pollutants may actually accumulate over time by dry deposition. During a small precipitation event, the resultant solution could he much more acidic than the rainfall itself. Specific systems (e.g., pothole communities, cryptobiotic soils, desert mountain aquatic systems) may not have the buffering capacity of surrounding rock and soil, and thus may be at greater risk to damage from acid deposition. 
Power plants and industrial facilities that add acidic pollutants to the atmosphere are abundant in the Southwest. The Colorado Plateau region, of particular interest for this study, is surrounded by coal- and oil-fired power plants (Fig. 1). A number of new sources are proposed on or near the Colorado Plateau in the next 20-50 years. Air masses from other industrialized areas (e.g. smelters in southern Arizond and northern Mexico, the Los Angeles Basin, etc.) are carried across the Colorado Plateau ${ }^{2}$. These polluted air masses can deposit hoth wet and dry acidic components on the Colorado Plateau. National Atmospheric Deposition Program (NADP) stations on or near the Colorado Plateau (Fig. 1) have recorded $\mathrm{pH}$ values of less than 5.0 for many weekly precipitation averages (the lowest was 3.84 at Mesa Verde National Park for the week of 30 Aug. 1983) in the past eight years ${ }^{3}$. A pH of 5.6 is generally considered to be "clean" precipitation ${ }^{4}$, although local conditions may affect what is accepted as "normal" pH in rainfall 5 . For example, natural mean annual $\mathrm{pH}$ in the Great Basin region has been estimated at about $6.0^{1}$.

Potholes are depressions in bedrock that are not in active drainages. Precipitation collected in these basins creates small, ephemeral aquatic environments. These pools contain fauna unique to temporary aquatic systems. Because aquatic organisms are particularly susceptible to decreased $\mathrm{pH}$ levels ${ }^{6-10}$, aquatic systems should be studied to determine the sensitivity of indigenous species. Although many surface waters in the Southwest are buffered, potholes generally have very low alkalinities ${ }^{1}$, and thus may be more susceptible to acid deposition. Biomonitoring of these sensitive species can then provide early indications that the system is being disturbed. Ephemeral pool ecosystems may show impacts to increased acid deposition before other systems in arid parks, and thus should be monitored. There is some evidence that despite low alkalinities in pothole water, there is a large reservoir of acid neutralizing capacity available in the sediment and/or the rock 11 .

Few studies have been made of pothole ecology or associated water chemistry on the Colorado Plateau12-14. Environmental tolerance ranges and hatching and mating stimulants are unknown for most species inhabiting Colorado Plateau potholes. Little is known about what determines patterns in community structure, what roles potholes play in surrounding terrestrial ecosystems, or what the impacts of various anthropogenic activities are on these relatively discrete ecosystems. The impacts of decreased pH or of increasing trace metal concentrations on pothole community and ecosystem processes are unknown, and should be investigated to determine the suitability of pothole systems for biomonitoring of acid deposition in the arid Southwest.

Pothole organisms are well adapted to living in the constantly changing, often harsh environment of ephemeral rock pools. Much of the year, potholes are dry; organisms have desiccation resistant (e.g. snails) or desiccation tolerant (e.g. crustacean eggs) stages, or they migrate to permanent water (e.g. backswimmers 15 ). Even during the wet phase, conditions can be severe. Temperatures can reach $35 \mathrm{deg}$. C, and then drop to $15 \mathrm{deg}$. C overnight. Photosynthesis can remove enough carbon dioxide to drive the $\mathrm{pH}$ from 6.5 to 9.4 in 6.5 hours 11 . Through a single wet phase, conductivity, average $\mathrm{pH}$, and alkalinity will 


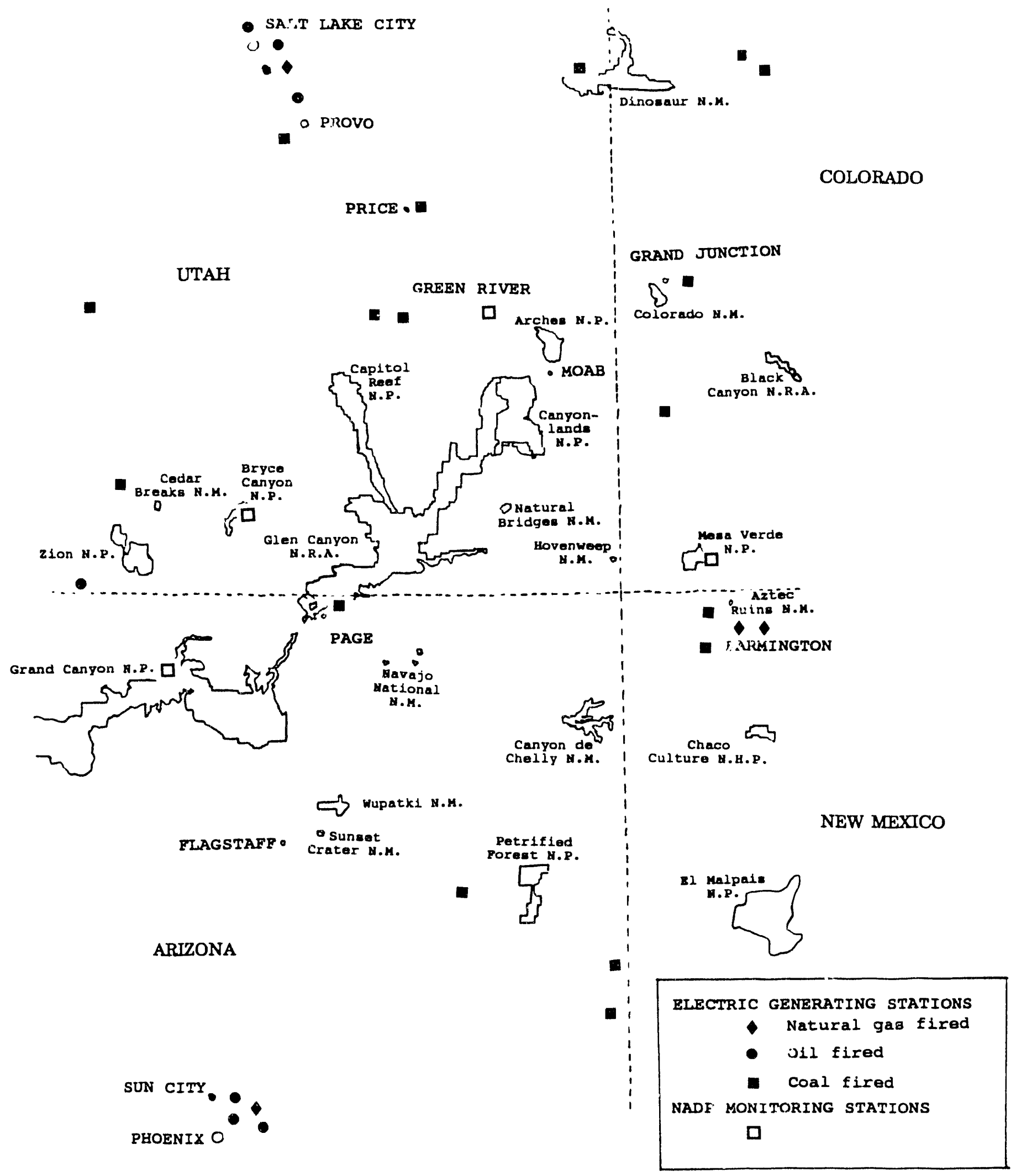

Figure 1. NPS units, electric generating stations, and NADP monitoring stations on or near the Colorado Plateau. 
increase while dissolved oxygen can decrease dramatically. Pothole inhabitants are adapted to a wide range of these environmental parameters, but conditions of their aquatic habitats still affect growth, reproductive efforts, and even survival $16-21$

A pothole is a very dynamic environment, but changes in pothole water as a result of acid depositioi may lie outside the natural range of conditions. Thus, lowered $\mathrm{pH}$, with or without an increase in heavy metal contamination, can upset the balance needed for pothole organisms to survive and reproduce successfully before the pool dries up.

In 1988 a study was initiated to address some of the questions concerning susceptibility of pothole ecosystems to acid deposition. Basic ecological questions of community structure and ecosystem functioning, as well as impacts of lowered $\mathrm{pH}$ on pothole systems were considered. As part of this study, water and sediment samples from selected potholes were analyzed for water quality parameters and trace element composition. The chemical composition of the water collected in these potholes is assumed to reflect an integration of the composition of precipitation and dry deposition falling directly into the pothole catchment basins and the composition of the sediments.

\section{EXPERIMENTAL METHODS}

\section{Water Chemistry:}

Water samples were collected from potholes in Grand County, Utah, during 1988 and 1989. Eleven potholes in Arches National Park and 15 in Grand Resource Area, BLM (hereafter referred to as BLM potholes or pools) were selected for each sample period, but logistics and/or field conditions (e.g. dry pools) limited the actual number sampled on any one date to between 3 and 20. Approximate locations of the sampling sites are shown in Fig. 2, and physical measuremerits presented in Table A-I in Appendix A. If a pothole scheduled to be sampled was dry, a nearby pool was selected as a substitute whenever possible. We did not correlate water chemistry of substitute potholes with originally selected potholes when both were wet due to funding constraints. Our primary objective was to investigate the general chemical composition of water in potholes shortly after rain occurred, rather than how the water chemistry of the same pothole behaved over a series of precipitation events. Sediment samples were collected from 10 of the 11 Arches pools that had previously had water samples taken, and from eight of the BLM pools (two pools still had water in them at sampling time) on 3 Nov. 1988.

In 1990 the study was extended in scope with water samples taken from selected potholes in the Lake Mead (LAME) National Recreation Area and Joshua Tree (JOTR) National Monument. This expansion was undertaken to determine whether any impact on water chemistry had occurred at sites that were nearer potential pollution sources (Navajo Generating Station, Mojave Generating Station, and Los Angeles Basin, respectively). 


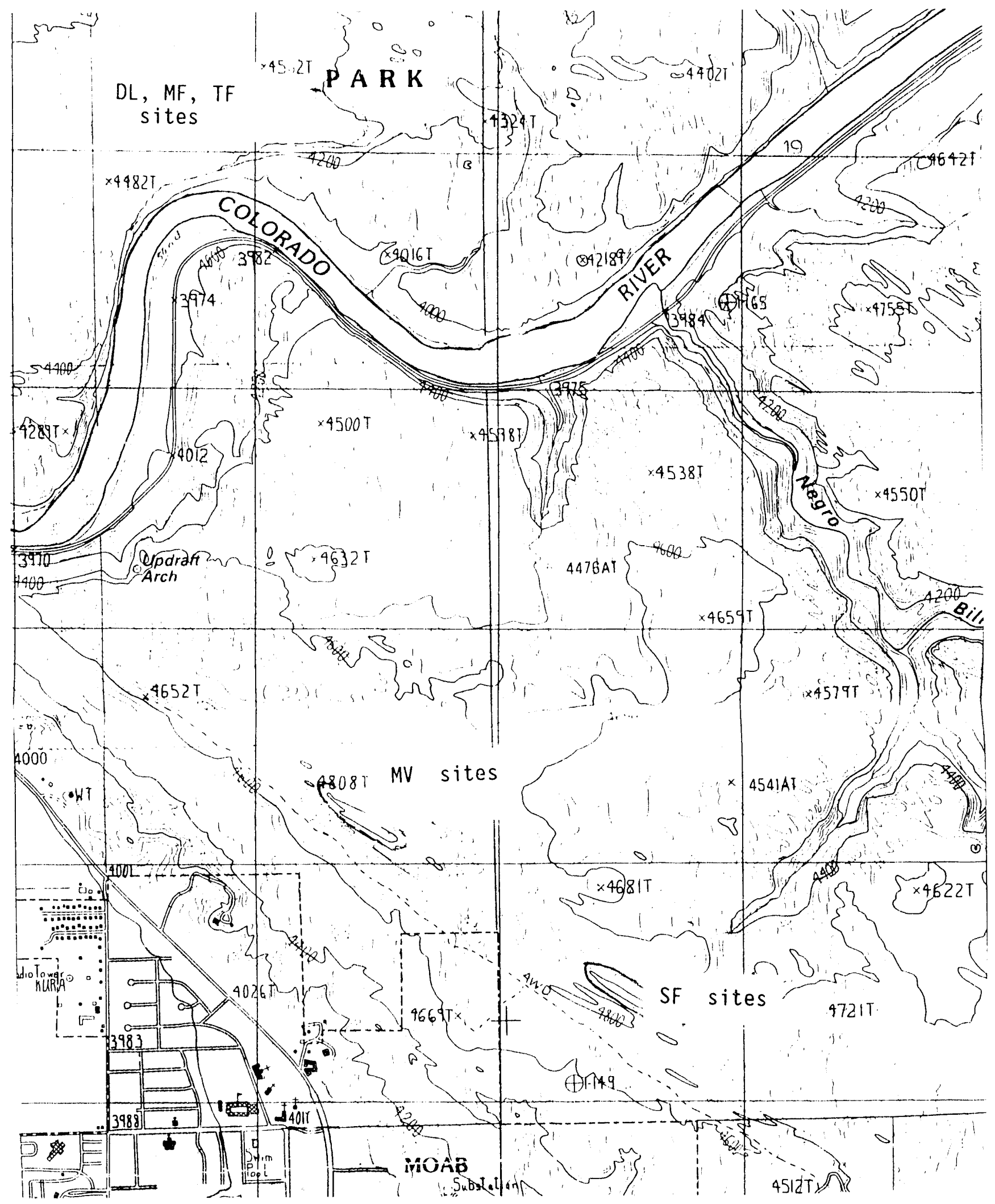

Figure 2. Sampling sites in Arches National Park and adjoining BLM land. 
The temperature and $\mathrm{pH}$ of each pool were measured in situ at every sampling period in 1989, using an Orion Model SA $230 \mathrm{pH}$ meter, with a Fisher combiration $\mathrm{pH}$ electrode with Calomel reference. A Beckman Series $10 \mathrm{pH}$ meter with temperature compensation and combination $\mathrm{pH}$ probes was used in 1990. Alkalinity in each pool was measured using a Hach alkalinity titration kit except for those taken on 28 July and 15 Sept. 1988 which did not have alkalinity measured. Due to the low alkalinities, $32 \mathrm{~mL}$ of sample was used for each titration instead of the $8 \mathrm{~mL}$ recommended by Hach. Bromocresol green/methyl red indicator was added to the sample and titrated to the color endpoint with $0.030 \mathrm{~N}$ sulfuric acid. The number of drops needed to reach the endpoint was multiplied by 4.275 to obtain alkalinity in $\mathrm{mg} / \mathrm{L}$ $\mathrm{CaCO}_{3}$. Some of these data were remeasured by acid titration to the methyl purple endpoint for total alkalinity (Method WI500) 22 after the samples had been received at Los Alamos.

\section{Trace Element Content:}

Water at all sites was collected in precleaned polyethylene bottles by pipetting two $100 \mathrm{~mL}$ samples in $5 \mathrm{~mL}$ increments from just below the surface of the pool using disposable pipettes. Great care was taken not to disturb the fine sediment. One sample was acidified with $5 \mathrm{~mL}$ of concentrated nitric acid (preservative for cation and trace metal analyses) and one sample remained untreated (alkalinity $a_{2}:$ anion analyses).

Anions $\left(\mathrm{F}^{-}, \mathrm{Cl}^{-}, \mathrm{NO}_{3}^{-}, \mathrm{SO}_{4}=\right.$, and $\mathrm{PO}_{4}=$ ) were determined by ion chromatography and major cations $\left(\mathrm{Na}^{+}, \mathrm{K}^{+}, \mathrm{Ca}^{2}+\right.$, and $\mathrm{Mg}^{2}+$ ) by flame atomic absorption or inductively coupled plasma atomic emission spectrometry. Other analyses included: conductivity by wheatstone bridge, alkalinity by titration, and up to 60 trace metals by inductively coupled plasma mass spectrometry (ICPMS). Details of thie chemical and instrumental methods employed may be found in Ref. 22. Quality assurance was maintained by concurrent analysis of a variety of National Institute of Standards and Technology (NIST, formerly the National Bureau of Standards) and Environmental Protection Agency (EPA) reference materials. Our quaiity assurance/control policies and techniques are detailed in Ref. 23.

Sediments:

Approximately $25 \mathrm{~g}$ of wet sediment at two different depths (surface and $1-2 \mathrm{~cm}$ below the surface) were collected. A polyethylene spoon was used to take the surface sample from the top centimeter of sediment over an area of approximately $25 \mathrm{~cm}^{2}$. Sediments from $1-2 \mathrm{~cm}$ depth were taken from the center of the area exposed by sampling surface material. All samples were packaged in polyethylene bags and shipped to Los Alamos.

In the laboratory, the samples were first air dried and then sieved through a 20 mesh brass screen to remove large debris. Instrumental neutron activation determination of up to 44 elements in each sample was performed at the Los Alamos National Laboratory's Omega West Reactor using the automated methods described in Refs. 24-25. Aliquots of approximately $4 \mathrm{~g}$ of soil were encapsulated in screw-capped polyethylene rabbits and irradiated in a thermal 
neutron flux of $6 \times 10^{12}$ neutrons $/ \mathrm{cm}^{2} / \mathrm{sec}$. The following is the irradiation and counting sequence currently employed:

1. First irradiation: $20 \mathrm{sec}$.

2. Transfer to delayed neutron counter, decay for $10 \mathrm{sec}$.

3. Count delayed neutrons for $30 \mathrm{sec}$.

4. Transfer to $\mathrm{Ge}(\mathrm{Li})$ detector, decay for $20 \mathrm{~min}$.

5. Count short-lived gamma-emitting activities for $475 \mathrm{sec}$.

6. Transfer back to reactor for a second irradiation of $500 \mathrm{sec}$.

7. Transfer to storage, decay for 4-7 days.

8. Transfer back to Ge(Li) detector, count for 1 hour.

9. Transfer back to storage, decay 2-3 weeks.

10. Transfer back to $\mathrm{Ge}(\mathrm{Li})$ detector for final count of 2 hours.

Every $\mathrm{Ge}(\mathrm{Li})$ detector is calibrated using NIST traceable materials. Initially, each detector is calibrated with a certified Eu-152 radiation source to establish relative efficiency and characteristic peak shape. Absolute calibration is achieved by running a large set of NIST Coal, Fly Ash, and Silicate Standard Reference Materials (SRMs). Quality assuranre for the automated neutron activation data reported in this work was provided by the concirrent analysis of geological SRMs produced by NIST that were submitted to the Reactor group as unknowns.

\section{RESULTS AND DISCUSSION}

Mean elemental and other constituent concentrations from unfiltered samples in groups of potholes are presented in Tables 1 and 2, with complete results of all chemical and physical measurements in pothole waters shown in Tables B-I through B-IV in Appendix B. The enrichment factor approach has again been employed to highlight elements that depart significantly from average crustal abundance in the pothole sediments from Grand County. This approach has been described in detail in Ref. 26. Mean enrichment factors are listed in Table 3 and the elemental concentration data and individual enrichment factors are given in Tables C-I through C-IV in Appendix C.

Temperature, pH, and alkalinity increase from sunrise to sunset each day in potholes 27. Increases in $\mathrm{pH}$ may be due to photosynthetic consumption of $\mathrm{CO}_{2}$. Therefore, some of the differences observed in these parameters are due to sampling at different times of day. The detailed compilations of these field data are presented in Tables D-I through D-III in Appendix D.

Among the "mineral" analyses $\left(\mathrm{F}^{-}, \mathrm{Cl}^{-}, \mathrm{NO}_{3}^{-}, \mathrm{SO}_{4}=\cdot \mathrm{Na}^{+}, \mathrm{K}^{+}, \mathrm{Ca}^{2}+\right.$, and $\left.\mathrm{Mg}^{2}{ }^{+}\right)$in the water samples, there seems to be a definite gradient with significant increases in many of the potholes nearest southern California for most of this groun of analytes. Among the trace 


\begin{tabular}{|c|c|c|c|c|c|c|c|c|c|c|c|}
\hline Site & $\begin{array}{c}A g \\
(u g / L)\end{array}$ & $\begin{array}{c}\text { Al } \\
\text { (ug/L) }\end{array}$ & $\begin{array}{c}\text { As } \\
(u g / L)\end{array}$ & $\begin{array}{c}\mathrm{Au} \\
(\mathrm{ug} / \mathrm{L})\end{array}$ & $\begin{array}{c}\text { B } \\
(u g / L)\end{array}$ & $\begin{array}{c}B a \\
(u g / L)\end{array}$ & $\begin{array}{c}\mathrm{Be} \\
(u g / L)\end{array}$ & $\begin{array}{c}B i \\
(u g / L)\end{array}$ & $\begin{array}{c}B r \\
(u g / L)\end{array}$ & $\begin{array}{c}\mathrm{Ca} \\
(\mathrm{mg} / \mathrm{L})\end{array}$ & $\begin{array}{c}\mathrm{Cd} \\
(\mathrm{ug} / \mathrm{L})\end{array}$ \\
\hline MV & $<1$ & $<10$ & $<10$ & $<1$ & $<100$ & 80 & $<10$ & $<1$ & $<100$ & 8.8 & $<1$ \\
\hline$S F$ & $<1$ & $<10$ & $<10$ & $<1$ & 30 & 100 & $<10$ & $<1$ & 110 & 18.0 & $<1$ \\
\hline$D L$ & $<1$ & $<10$ & $<10$ & $<1$ & $<100$ & 77 & $<10$ & $<1$ & 100 & 11.4 & $<1$ \\
\hline MF & $<1$ & $<10$ & $<10$ & $<1$ & $<100$ & 80 & $<10$ & $<1$ & $<100$ & 9.0 & $<1$ \\
\hline TF & $<1$ & $<10$ & $<10$ & $<1$ & $<100$ & 90 & $<10$ & $<1$ & 110 & 13.7 & $<1$ \\
\hline LAME & $<1$ & $\cdots$ & $<10$ & $<1$ & $<100$ & $<100$ & $<10$ & $<1$ & $<100$ & 13.0 & $<0.1$ \\
\hline JOTR & $<1$ & $\cdots$ & $<10$ & $<1$ & 1600 & $<100$ & $<10$ & $<1$ & $<100$ & 26.0 & $<0.1$ \\
\hline
\end{tabular}

\begin{tabular}{|c|c|c|c|c|c|c|c|c|c|c|c|}
\hline site & $\begin{array}{c}\mathrm{Ce} \\
(u g / L)\end{array}$ & $\begin{array}{c}C l \\
(m g / L)\end{array}$ & $\begin{array}{c}\text { Co } \\
(u g / L)\end{array}$ & $\begin{array}{c}\mathrm{Cr} \\
(\mathrm{ug} / \mathrm{L})\end{array}$ & $\begin{array}{c}\text { Cs } \\
(\mathrm{ug} / \mathrm{L})\end{array}$ & $\begin{array}{l}\mathrm{Cu} \\
(\mathrm{ug} / \mathrm{L} .)\end{array}$ & $\begin{array}{c}\text { Dy } \\
(\text { ug/L) }\end{array}$ & $\begin{array}{c}E r \\
(u g / L)\end{array}$ & $\begin{array}{c}E U \\
(U g / L)\end{array}$ & $\begin{array}{c}F \\
(m g / L)\end{array}$ & $\begin{array}{l}\mathrm{Fe} \\
(\mathrm{mg} / \mathrm{L})\end{array}$ \\
\hline MV & $<1$ & 1.0 & $<1$ & $<10$ & $<1$ & $<1$ & $<1$ & $<1$ & $<1$ & $<0.2$ & $<0.5$ \\
\hline$S F$ & $<1$ & 1.7 & $<1$ & $<10$ & $<1$ & $<50$ & $<1$ & $<1$ & $<1$ & 0.3 & 1.0 \\
\hline DL & $<1$ & 1.8 & $<1$ & $<10$ & $<1$ & $<30$ & $<1$ & $<1$ & $<1$ & $<0.2$ & 3.2 \\
\hline MF & $<1$ & 1.7 & $<1$ & $<10$ & $<1$ & 70 & $<1$ & $<1$ & $<1$ & $<0.2$ & 3.0 \\
\hline$T F$ & $<1$ & 2.1 & $<1$ & $<10$ & $<1$ & $<20$ & $<1$ & $<1$ & $<1$ & $<0.3$ & 2.0 \\
\hline LAME & $<1$ & 4.2 & $<1$ & 2.3 & $<1$ & 6.8 & $<1$ & $<1$ & $<1$ & $<0.2$ & $<0.5$ \\
\hline JOTR & $<1$ & 14.8 & $<1$ & 4.1 & $<1$ & 13 & $<1$ & $<1$ & $<1$ & $<0.2$ & 0.6 \\
\hline
\end{tabular}

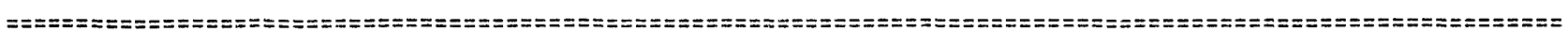

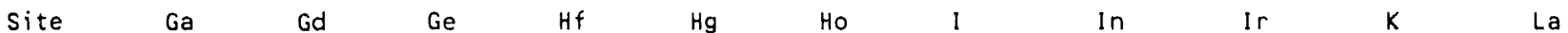

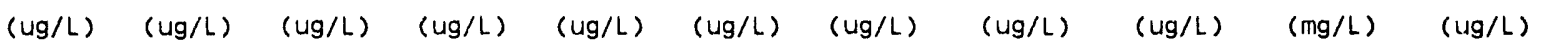

\begin{tabular}{|c|c|c|c|c|c|c|c|c|c|c|c|}
\hline MV & $<1$ & $<1$ & $<1$ & $<1$ & $<1$ & $<1$ & $<1$ & $<1$ & $<1$ & 1.2 & $<1$ \\
\hline SF & $<1$ & $<1$ & $<1$ & $<1$ & $<1$ & $<1$ & $<1$ & $<1$ & $<1$ & 1.0 & $<1$ \\
\hline DL & $<1$ & $<1$ & $<1$ & $<1$ & $<1$ & $<1$ & $<2$ & $<1$ & $<1$ & 1.4 & $<1$ \\
\hline MF & $<1$ & $<1$ & $<1$ & $<1$ & $<1$ & $<1$ & $<5$ & $<1$ & $<1$ & 1.9 & $<1$ \\
\hline TF & $<1$ & $<1$ & $<1$ & $<1$ & $<1$ & $<1$ & $<3$ & $<1$ & $<1$ & 1.4 & $<1$ \\
\hline LAME & $<1$ & $<1$ & $<1$ & $<1$ & $<1$ & $<1$ & $<1$ & $<1$ & $<1$ & 1.5 & $<1$ \\
\hline JOTR & $<1$ & $<1$ & $<1$ & $<1$ & $<1$ & $<1$ & $<4$ & $<1$ & $<1$ & 1.9 & $<1$ \\
\hline
\end{tabular}

\begin{tabular}{|c|c|c|c|c|c|c|c|c|c|c|}
\hline Site & $\begin{array}{c}L i \\
(u g / L)\end{array}$ & $\begin{array}{c}\mathrm{Lu} \\
(u g / L)\end{array}$ & $\begin{array}{c}\mathrm{Mg} \\
(\mathrm{mg} / \mathrm{L})\end{array}$ & $\begin{array}{c}M n \\
(u g / L)\end{array}$ & $\begin{array}{c}\text { Mo } \\
(u g / L)\end{array}$ & $\begin{array}{c}\mathrm{Na} \\
(\mathrm{mg} / \mathrm{L})\end{array}$ & $\begin{array}{c}\mathrm{Nb} \\
(\mathrm{ug} / \mathrm{L})\end{array}$ & $\begin{array}{c}\text { Nd } \\
(u g / L)\end{array}$ & $\begin{array}{c}N i \\
(u g / L)\end{array}$ & $\begin{array}{c}\text { Os } \\
(u g / L)\end{array}$ \\
\hline MV & $<10$ & $<1$ & 1.1 & 59 & $<1$ & 0.8 & $<1$ & $<1$ & $<50$ & $<1$ \\
\hline SF & $<10$ & $<1$ & 1.3 & 150 & $<1$ & 0.6 & $<1$ & $<1$ & $<50$ & $<1$ \\
\hline$D L$ & $<10$ & $<1$ & 1.1 & 220 & $<1$ & 1.2 & $<1$ & $<1$ & $<50$ & $<1$ \\
\hline MF & $<10$ & $<1$ & 1.1 & 80 & $<1$ & 1.2 & $<1$ & $<1$ & 70 & $<1$ \\
\hline TF & $<10$ & $<1$ & 1.2 & 180 & $<1$ & 1.3 & $<1$ & $<1$ & 110 & $<1$ \\
\hline LAME & $<100$ & $<1$ & 2.0 & 17 & $<1$ & 1.3 & $<1$ & $<1$ & $<1$ & $<1$ \\
\hline JOTR & 250 & $<1$ & 2.3 & 8 & $<2$ & 11.9 & $<1$ & $<1$ & $<3$ & $<1$ \\
\hline
\end{tabular}




\begin{tabular}{|c|c|c|c|c|c|c|c|c|c|c|}
\hline Site & $\begin{array}{c}\mathrm{Pb} \\
(u g / L)\end{array}$ & $\begin{array}{c}P d \\
(u g / L)\end{array}$ & $\begin{array}{c}\mathrm{Pr} \\
(u g / L)\end{array}$ & $\begin{array}{c}P t \\
(u g / L)\end{array}$ & $\begin{array}{c}R b \\
(u g / L)\end{array}$ & $\begin{array}{c}\operatorname{Re} \\
(u g / L)\end{array}$ & $\begin{array}{c}R h \\
(u g / L)\end{array}$ & $\begin{array}{c}R u \\
(u g / L)\end{array}$ & $\begin{array}{c}S b \\
(u g / L)\end{array}$ & $\begin{array}{c}\text { Sc } \\
(u g / L)\end{array}$ \\
\hline MV & $<1$ & $<1$ & $<1$ & $<1$ & $<1$ & $<1$ & $<1$ & $<1$ & $<1$ & $<1$ \\
\hline SF & $<2$ & $<1$ & $<1$ & $<1$ & $<1$ & $<1$ & $<1$ & $<1$ & $<1$ & $<1$ \\
\hline$D L$ & $<2$ & $<1$ & $<\uparrow$ & $<1$ & $<1$ & $<1$ & $<1$ & $<1$ & $<1$ & $<1$ \\
\hline MF & $<2$ & $<1$ & $<1$ & $<1$ & $<1$ & $<1$ & $<1$ & $<1$ & $<1$ & $<1$ \\
\hline$T F$ & $<2$ & $<1$ & $<1$ & $<1$ & $<1$ & $<1$ & $<1$ & $<1$ & $<1$ & $<1$ \\
\hline LAME & $<2$ & $<1$ & $<1$ & $<1$ & $<1$ & $<1$ & $<1$ & $<1$ & $<1$ & $<1$ \\
\hline JOTR & 12 & $<1$ & $<1$ & $<1$ & $<1$ & $<1$ & $<1$ & $<1$ & $<1$ & $<1$ \\
\hline
\end{tabular}

\begin{tabular}{|c|c|c|c|c|c|c|c|c|c|}
\hline site & $\begin{array}{c}\text { Se } \\
(u g / L)\end{array}$ & $\begin{array}{c}S m \\
(u g / L)\end{array}$ & $\begin{array}{c}S n \\
(u g / L)\end{array}$ & $\begin{array}{c}S r \\
(u g / L)\end{array}$ & $\begin{array}{c}\text { Ta } \\
(u g / L)\end{array}$ & $\begin{array}{c}\text { Tb } \\
(u g / L)\end{array}$ & $\begin{array}{c}\text { Te } \\
(u g / L)\end{array}$ & $\begin{array}{c}\text { Th } \\
(u g / L)\end{array}$ & $\begin{array}{c}\mathrm{Ti} \\
(\mathrm{ug} / \mathrm{L})\end{array}$ \\
\hline MV & $<10$ & $<1$ & $<1$ & 110 & $<1$ & $<1$ & $<1$ & $<1$ & $<10$ \\
\hline SF & $<20$ & $<1$ & $<1$ & 220 & $<1$ & $<1$ & $<1$ & $<1$ & $<10$ \\
\hline$D L$ & 30 & $<1$ & $<1$ & 340 & $<1$ & $<1$ & $<1$ & $<1$ & $<10$ \\
\hline$M F$ & 24 & $<1$ & $<1$ & 350 & $<1$ & $<1$ & $<1$ & $<1$ & $<10$ \\
\hline TF & 35 & $<1$ & $<1$ & 270 & $<1$ & $<1$ & $<1$ & $<1$ & $<10$ \\
\hline LAME & $<10$ & $<1$ & $<1$ & 72 & $<1$ & $<1$ & $<1$ & $<1$ & $<10$ \\
\hline JOTR & $<10$ & $<1$ & 3 & 130 & $<1$ & $<1$ & $<1$ & $<1$ & $<10$ \\
\hline
\end{tabular}

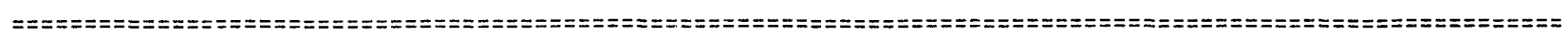

\begin{tabular}{|c|c|c|c|c|c|c|c|c|c|}
\hline Site & $\begin{array}{c}T l \\
(u g / L)\end{array}$ & $\begin{array}{c}\mathrm{Tm} \\
(\mathrm{ug} / \mathrm{L})\end{array}$ & $\begin{array}{c}U \\
(u g / L)\end{array}$ & $\begin{array}{c}V \\
(u g / L)\end{array}$ & $\begin{array}{c}W \\
(u g / L)\end{array}$ & $\begin{array}{c}Y \\
(u g / L)\end{array}$ & $\begin{array}{c}Y b \\
(u g / L)\end{array}$ & $\begin{array}{c}\mathrm{Zn} \\
(\mathrm{ug} / \mathrm{L})\end{array}$ & $\begin{array}{c}2 r \\
(u g / L)\end{array}$ \\
\hline MV & $<1$ & $<1$ & $<1$ & $<1$ & $<1$ & $<1$ & $<1$ & $<1$ & $<1$ \\
\hline$S F$ & $<1$ & $<1$ & $<2$ & 2 & $<1$ & $<1$ & $<1$ & $<10$ & $<1$ \\
\hline DL & $<1$ & $<1$ & $<2$ & $<1$ & $<1$ & $<1$ & $<1$ & $<10$ & $<1$ \\
\hline MF & $<1$ & $<1$ & $<2$ & $<1$ & $<1$ & $<1$ & $<1$ & $<10$ & $<1$ \\
\hline TF & $<1$ & $<1$ & $<2$ & $<1$ & $<1$ & $<1$ & $<1$ & $<10$ & $<1$ \\
\hline LAME & $<1$ & $<1$ & $<1$ & $<1$ & $<1$ & $<1$ & $<1$ & 22 & $<1$ \\
\hline JOTR & $<1$ & $<1$ & $<1$ & 2 & $<1$ & $<1$ & $<1$ & 14 & $<1$ \\
\hline
\end{tabular}




\begin{tabular}{|c|c|c|c|c|c|c|c|c|}
\hline Site & $\begin{array}{c}\text { Conductivity } \\
\text { (microhos) }\end{array}$ & $\begin{array}{c}\mathrm{NO}_{3}-\mathrm{N} \\
(\mathrm{mg} / \mathrm{L})\end{array}$ & $\begin{array}{l}\text { Field pH } \\
\text { (units) }\end{array}$ & $\begin{array}{l}\mathrm{PO}_{4}-\mathrm{P} \\
(\mathrm{mg} / \mathrm{L})\end{array}$ & $\underset{(\mathrm{mg} / \mathrm{L})}{\mathrm{SO}_{4}}$ & $\begin{array}{c}\text { Total Alk } \\
\text { (field) }\end{array}$ & $\begin{array}{c}\text { kalinity (mg/L) } \\
\text { (laboratory) }\end{array}$ & $\begin{array}{c}\text { Temperature } \\
\text { (deg. C) }\end{array}$ \\
\hline MV & 61 & $<0.2$ & 8.0 & $<0.2$ & 7.6 & 26 & 10 & 25.4 \\
\hline SF & 108 & $<0.2$ & 7.9 & $<0.2$ & 10.9 & 36 & 24 & 21.7 \\
\hline $\mathrm{DL}$ & 87 & $<0.4$ & 7.5 & $<0.2$ & 14.2 & 28 & 16 & 27.0 \\
\hline MF & 84 & 0.4 & 8.2 & $<0.2$ & 12.4 & 26 & 19 & 25.4 \\
\hline TF & 91 & 0.6 & 8.3 & $<0.2$ & 17.2 & 28 & 21 & 29.0 \\
\hline LAME & -.- & ... & 9.1 & $\ldots$ & 25 & 25 & 25 & 24.4 \\
\hline JOTR & --- & 15.4 & 9.4 & 0.4 & 32 & 26 & ... & 27.0 \\
\hline
\end{tabular}

Notes: field $=$ measurements made in situ laboratory $=$ measurements made in Los Alamos

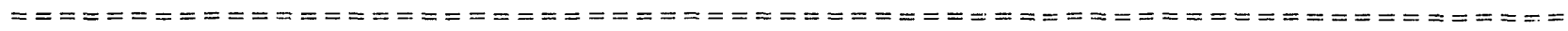
Table 3: Summary of Enrichment Factors for Grand County, Utah, Pothole Sediments

\begin{tabular}{|c|c|c|c|c|c|c|c|}
\hline Group & $\begin{array}{l}\text { Depth } \\
(\mathrm{cm})\end{array}$ & $\begin{array}{l}\text { Samp } \\
\text { (no.) }\end{array}$ & As & $\mathrm{Ba}$ & $\mathrm{Br}$ & $\mathrm{Ca}$ & $\mathrm{Co}$ \\
\hline \multirow[t]{2}{*}{ MV } & 0 & 3 & $13 \pm 4$ & $2.1 \pm 0.6$ & $3.5 \pm 1.9$ & $0.3 \pm 0.2$ & $0.8 \pm$ \\
\hline & $1-2$ & 3 & $10 \pm 4$ & $2.2 \pm 0.2$ & $0.8 \pm 0.2$ & 0.1 & $0.5 \pm 0.1$ \\
\hline \multirow[t]{2}{*}{ SF } & 0 & 5 & $12 \pm 3$ & $1.6 \pm 0.4$ & $3.6 \pm 2.2$ & $0.2 \pm 0.1$ & $0.9 \pm 0.2$ \\
\hline & $1-2$ & 6 & $8 \pm 1$ & $2.3 \pm 0.3$ & $1.5 \pm 0.6$ & $\overline{0.1}$ & $0.7 \mp 0.2$ \\
\hline \multirow[t]{2}{*}{ DL } & 0 & 2 & $\overline{8.2}$ & $\overline{1.4}$ & $4 . \overline{0}$ & 0.3 & $1 . \overline{0}$ \\
\hline & $1-2$ & 2 & 6.3 & 2.3 & 2.2 & 0.1 & 0.0 \\
\hline \multirow[t]{2}{*}{$\mathrm{MF}$} & 0 & 3 & $6 \pm 1$ & $1.9 \pm 0.4$ & $1.9 \pm 0.7$ & 0.2 & $0.5 \pm 0.1$ \\
\hline & $1-2$ & 3 & $6 \pm 1$ & $2.6 \pm 0.4$ & $1.4 \pm 0.2$ & 0.1 & $0.5 \pm 0.1$ \\
\hline \multirow[t]{2}{*}{$\mathrm{TF}$} & 0 & 5 & $5 \pm 3$ & $1.7 \pm 0.3$ & $3.4 \pm 1.0$ & $0.2 \pm 0.1$ & $0.7 \pm 0$ \\
\hline & $1-2$ & 5 & $6 \pm 2$ & $2.4 \pm 0.5$ & $1.4 \pm 0.5$ & $\overline{0.1}$ & $0.6 \mp 0$ \\
\hline
\end{tabular}

\begin{tabular}{|c|c|c|c|c|c|c|c|}
\hline Group & $\begin{array}{c}\text { Depth } \\
\text { (cm) }\end{array}$ & $\begin{array}{l}\text { Samp } \\
\text { (no.) }\end{array}$ & Cs & $\mathrm{Eu}$ & $\mathrm{Fe}$ & $\mathrm{Mg}$ & $\mathrm{Mn}$ \\
\hline \multirow[t]{2}{*}{ MV } & 0 & 3 & $1.8 \pm 0.1$ & $1.7 \pm 0.1$ & $0.6 \pm 0.1$ & $0.5 \pm 0.1$ & $1.5 \pm 0.6$ \\
\hline & $1-2$ & 3 & $1.4 \pm 0.1$ & $1.3 \pm 0.2$ & $0.5 \pm 0.1$ & $0.3 \pm 0.1$ & $0.3 \pm 0.1$ \\
\hline \multirow[t]{2}{*}{ SF } & 0 & 5 & $1.6 \pm 0.1$ & $1.6 \pm 0.3$ & $0.7 \pm 0.1$ & $0.6 \pm 0.1$ & $2.3 \pm 1.4$ \\
\hline & $1-2$ & 6 & $1.6 \pm 0.2$ & $1.4 \pm 0.1$ & $0.5 \pm 0.2$ & $0.4 \pm 0.1$ & $0.3 \pm 0.1$ \\
\hline \multirow[t]{2}{*}{ DL } & 0 & 2 & $\overrightarrow{1.6}$ & 1.9 & 0.7 & $0 . \overline{0.5}$ & $\overline{1.4}$ \\
\hline & $1-2$ & 2 & 1.6 & 1.4 & 0.5 & 0.3 & 0.2 \\
\hline \multirow[t]{2}{*}{$\mathrm{MF}$} & 0 & 3 & $1.4 \pm 0.1$ & $1.4 \pm 0.1$ & $0.5 \pm 0.1$ & $0.4 \pm 0.1$ & $0.6 \pm 0.1$ \\
\hline & $1-2$ & 3 & $1.6 \pm 0.2$ & $1.4 \pm 0.2$ & $0.5 \pm 0.1$ & $0.3 \pm 0.1$ & $0.2 \pm 0.1$ \\
\hline \multirow[t]{2}{*}{$\mathrm{TF}$} & 0 & 5 & $1.5 \pm 0.2$ & $1.5 \pm 0.2$ & $0.6 \pm 0.1$ & $0.4 \pm 0.1$ & $1.1 \pm 0.3$ \\
\hline & $1-2$ & 5 & $1.5 \pm 0.2$ & $1.3 \pm 0.2$ & $0.5 \pm 0.1$ & $0.3 \pm 0.1$ & $0.3 \pm 0.2$ \\
\hline
\end{tabular}




\begin{tabular}{|c|c|c|c|c|c|c|c|c|}
\hline Group & $\begin{array}{c}\text { Depth } \\
(\mathrm{cm})\end{array}$ & $\begin{array}{l}\text { Samp } \\
\text { (No.) }\end{array}$ & $\mathrm{Sb}$ & $\mathrm{Sc}$ & $\mathrm{Se}$ & $\mathrm{U}$ & $\mathrm{V}$ & $\mathrm{Zn}$ \\
\hline \multirow[t]{2}{*}{ MV } & 0 & 3 & $25 \pm 5$ & $0.7 \pm 0.1$ & $57 \pm 11$ & $1.9 \pm 0.8$ & $0.8 \pm 0.1$ & $1.5 \pm 0.3$ \\
\hline & $1-2$ & 3 & $25 \pm 3$ & $0.5 \pm 0.1$ & $60 \pm 30$ & $2.0 \pm 0.8$ & $0.6 \pm 0.2$ & 0.7 \\
\hline \multirow[t]{2}{*}{ SF } & 0 & 5 & $21 \pm 2$ & $0.8 \pm 0.2$ & $68 \pm 9$ & $2.1 \pm 0.3$ & $1.0 \pm 0.2$ & $1.5 \pm 0.2$ \\
\hline & $1-2$ & 6 & $22 \mp 4$ & $0.6 \mp 0.2$ & $77 \mp 22$ & $2.2+0.4$ & $0.7 \mp 0.1$ & $1.6 \overline{+0.4}$ \\
\hline \multirow[t]{2}{*}{$\mathrm{DL}$} & 0 & 2 & $\overline{16}$ & $\overline{0.8}$ & 50 & $\overline{3.8}$ & $\overline{1.2}$ & $\overline{1.7}$ \\
\hline & $1-2$ & 2 & 17 & 0.6 & 102 & 2.9 & 0.8 & 1.4 \\
\hline \multirow[t]{2}{*}{ MF } & 0 & 3 & $17 \pm 1$ & $0.6 \pm 0.2$ & $46 \pm 6$ & $1.9 \pm 0.6$ & $1.0 \pm 0.4$ & $0.9 \pm 0.2$ \\
\hline & $1-?$ & 3 & $21 \pm 1$ & $0.5 \pm 0.1$ & $86 \pm 38$ & $2.1 \pm 0.2$ & $0.6 \pm 0.1$ & $1.4 \pm 0.3$ \\
\hline \multirow[t]{2}{*}{$\mathrm{TF}$} & 0 & 5 & $18 \pm 1$ & $0.7 \pm 0.1$ & $42 \pm 8$ & $3.1 \pm 1.3$ & $1.0 \pm 0.3$ & $1.2 \pm 0.3$ \\
\hline & $1-2$ & 5 & $18 \pm 3$ & $0.5 \pm 0.1$ & $79 \pm 30$ & $2.7 \pm 0.9$ & $0.7 \pm 0.1$ & $1.2 \pm 0.4$ \\
\hline
\end{tabular}

metals in waters, $\mathrm{Cu}, \mathrm{Pb}, \mathrm{V}$, and $\mathrm{Zn}$ are definitely enriched $\mathrm{ir}$. TOTR relative to LAME ard Grand County, Utah. These are common industrial and energy production/consumption related trace elements. Boron is also strongly enriched in all the JOTR water samples relative to all the other sites. This element is commonly emitted from both coal-fired power plants and automobiles using certain types of unleaded gasoline. We consider these enrichments to be evidence of wet and dry deposicion from long-range transport of atmospheric aerosols generated in southern California. Git the entire suite of elements/anions determined in the water samples, only iron and manganese were elevated in the Grand County samples relative to those closer tc urban areas, and may represent the enrichment and mobilization of $\mathrm{Fe}$ and $\mathrm{Mn}$ in the desert jouthwest ecosystem due to cryptobiotic soil activity. The zinc data from 21 Aug. 1988 in Grand County are uniformly high, and probably represent contaminared samples due to inexperience with the sampling equipment and the concentrated nitric acid preservative.

The enrichment factors in the Grand County sediments presented in Table 3 reflect the common features of unpolluted soils in the desert Southwest. Arsenic, bromine, antimony, selenium, and uranium are significantly enriched relative to average crustal materials.

Calcium and magnesium are definitely depleted, while the remaining elements have enrichment factors near 1.0 indicating little difference from mean crustal abundance. It is noteworthy that although $\mathrm{Mn}$ was measured at elevated concentrations in the pothole waters, this was not reflected in the sediments. Unfortunately, no sediment samples were collected from LAME or JOTR for comparison.

\section{SUMMARY AND MANAGEMENT IMPLICATIONS}

The vast majority of trace elemental concentrations in pothole waters in all areas are below the detection limits of our most sensitive instrumentation. Most of these will be present in the waters at the sub-parts-per-billion range $(\operatorname{microgram} / \mathrm{L})$. This represents very clean waters 
showing little impact from metal-rich airborne particulate matter from anthropogenic sources. At the present time, the Grand County potholes provide excelleni background data against which more urbanized catchment basins can be evaluated. Many of the mineral analytes $(\mathrm{Ca}$, $\mathrm{Cl}, \mathrm{Na}, \mathrm{NO}_{3}-\mathrm{N}$, and $\left.\mathrm{SO}_{4}\right)$ and several trace metals $(\mathrm{B}, \mathrm{Cu}, \mathrm{Pb}, \mathrm{V}$, and $\mathrm{Zn}$ ) suggest input from some external source at JOTR.

The limited pothole data presented in this study suggest that these widespread desert features may indeed be used to assess long-term trends in atmospheric deposition in remote ecosystems. At present, no impact on the biological resource in these rock pools has been observed.

\section{ACKNOWLEDGMENTS}

We thank William Burke and Mark Heuston for collecting samples in LAME and JOTR, respectively; Nathan Webb, Charles Schultz, Terry Knouff, Amy Smith, and Alison Dunlap for assisting with the Arches and BLM sample collection; the staff of the Los Alamos Omega West Reactor for their assistance with the neutron activation analysis of the sediments; and Ken Stolte of the NPS Air Quality Division for his technical interest and program management support during all phases of the project.

\section{REFERENCES}

(1) P. Roth, C. Blanchard, J. Harte, H. Michaels, and M. T. El-Ashry, The American West's Acid Rain Test, World Resources Institute Res. Rep. 1 (1985).

(2) W. M. Malm and D. B. Joseph, "Regional Haze in National Parks in the West, Animated Video Modeling of Pollution Data in the Southwestern United States, " National Park Service, Air Quality Division (1985).

(3) NADP/NTN Database, "Semi-Annual Precipitation Chemistry Data, National Atmospheric Deposition Program," Natural Resource Ecology Laboratory, Colorado State University, Ft. Collins, CO (1991).

(4) R. J. Charlson and H. Rodhe, "Factors Controlling the Acidity of Natural Rainwater," Nature, 295: 683-685 (1982).

(5) R. Sequeira, "Acid Rain: An Assessment Based on Acid-Base Considerations, " J. Air Pollut. Control Assoc., 32: 241-244 (1982).

(6) J. M. Eilers, G. J. Lein, and R. G. Berg, "Aquatic Organisms in Acidic Environments: A Literature Review," Wisconsin Dept. of Natural Resources Technical Bulletin No. 150 (1984). 
(7) L. Arvola, K. Salonen, I. Bergstrom, A. Heinanen, and A. Ojala, "Effects of Experimental Acidification on Photo-, Bacterio-, and Zoo-Plankton in Enclosures of a Highly Humic Lake", Int. Revue Ges. Hydrobiol., 71: 737-758 (1986).

(8) S. J. Ormerod, P. Boole, C. P. McCahon, N. S. Weatherley, D. Pascoe, and R. W. Edwards, "Short-Term Experimental Acidification of a Welsh Stream: Comparing the Biological Effects of Hydrogen Ions and Aluminum," Freshwater Biology, 17: 341-356 (1987).

(9) B. A. Pierce and N. Sikand, "Variation in Acid Tolerance of Connecticut Wood Frogs: Genetic and Maternal Effects," Can. J. Zool., 63: 1647-1651 (1985).

(10) N. S. Weatherley and S. J. Oremrod, "The Impact of Acidification on Micro Invertebrate Assemblages in Welsh Streams: Towards an Empirical Model," Environ. Pollut., 46: 223-240 (1987).

(11) T. Graham, National Park Service, private communication of unpublished data (1992).

(12) S. Romney, "Bionomics of Aedes pactius neilsen, the Rock Pool Mosquito," Ph.D. dissertation, University of Utah, Salt Lake City, UT (1971).

(13) R. Findley, "Miracle of the Potholes," National Geographic, 148(4): $570-579$ (1975).

(14) S. I. Dodson, "Animal Assemblages in Temporary Desert Rock Pools: Aspects of the Ecology of Dasyhelea subletiei (Diptera: Ceratopogonidae), "J. N. Am. Benthol. Soc, 6: 6571 (1987).

(15) D. Belk and G. A. Cole, "Adaption Biology of Desert Temporary-Pond Inhabitants," in Environmental Physiology of Desert Organisms, N. F. Hadley (ed.), Dowden, Hutchinson, and Ross, Stroudsburg, Pa., pp. 207-226 (1975).

(16) F. Horne, "Effects of Physical-Chemical Factors on the Distribution and Occurrence of Some Southeastern Wyoming Phyllopods," Ecology, 48: 472-477 (1967).

(17) L. R. Brown and L. H. Carpelan, "Egg Hatchings and Life History of a Fairy Shrimp Branchinecta mackini dexter (Crustacea: Anostraca) in a Mojave Desert Playa (Rabbit Dry Lake)," Ecology, 52: 41-54 (1971).

(18) F. R. Horne, "Some Effects of Temperature and Oxygen Concentration on Phyllopod Ecology," Ecology, 52: 343-347 (1971). 
(19) S. D. Hillyard and A. Vinegar, "Respiration and Thermal Tolerance of the Phyllopod Crustacea Triops longicanudatus and Thamnocephalus platyurus Inhabiting Desert Ephemeral Ponds," Physiological Zoology, 45: 189-195 (1972).

(20) W. J. O'Brien and F. DeNoyelles, Jr., "Photosynthetically Elevated pH as a Factor in Zoo Plankton Mortality in Nutrient Enriched Ponds, "Ecology, 53: 605-614 (1972).

(21) D. B. Donald, "Erratic Occurrence of Aanostracans in a Temporary Pond: Acolonization and Extinction or Adaption to Variations in Annual Weather?, "Canadian Journal of Zoology, 61: $1492-1498(1983)$.

(22) M. A. Gautier and E. S. Gladney (eds.), "Health and Environmental Chemistry: Analytical Techniques, Data Management, and Quality Assurance," Los Alamos National Laboratory report LA-10300-M, Vols. I and II (1987).

(23) M. A. Gautier, E. S. Gladney, M. B. Phillips, and B. T. O'Malley, "Quality Assurance for Health and Environmental Chemistry: 1987, " Los Alamos National Laboratory report I 4-11454-MS (1988).

(24) E. S. Gladney, D. B. Curtis, D. R. Perrin, J. W. Owens, and W. E. Goode, "Nuclear Techniques for the Chemical Analysis of Environmental Materials, "Los Alamos Scientific Laboratory Report LA-8192-MS (1980).

(25) S. R. Garcia, W. K. Hensley, M. M. Minor, M. M. Denton, and M. A. Fuka, "An Automated Multidetector System for Instrumental Neutron Activation Analysis of Geological and Environmental Materials," in Atomic and Nuclear Methods in Fossil Energy Research, R. H. Filby, B. S. Carpenter, and R. C. Ragaini, eds., pp. 133-140 (1982).

(26) E. S. Gladney, "Origins and Effects of Dry-Deposited Materials in Desert Ecosystems: Some Atmospheric Chemistry Considerations", in Acid Rain and Air Pollution in Desert Park Areas, National Park Service Technical Report NPS/NRAQD/NRTR-91/02, pp. 52-67 (1991).

(27) T. Graham, National Park Service, unpublished data (1989). 
Appendix A: Physical Characteristics of Potholes

$-15-$ 


\begin{tabular}{|c|c|c|c|c|c|c|c|}
\hline $\begin{array}{c}\text { site } \\
\text { No. }\end{array}$ & $\begin{array}{l}\text { Maximum } \\
\text { Aręa } \\
\left(m^{2}\right)\end{array}$ & $\begin{array}{l}\text { Maximum } \\
\text { Depth } \\
(\mathrm{cm})\end{array}$ & $\begin{array}{l}\text { Maximum } \\
\text { volume } \\
\text { (L) }\end{array}$ & $\begin{array}{c}\text { site } \\
\text { No. }\end{array}$ & $\begin{array}{l}\text { Maximum } \\
\text { Aręa } \\
\left(m^{2}\right)\end{array}$ & $\begin{array}{l}\text { Maximum } \\
\text { Depth } \\
(\mathrm{cm})\end{array}$ & $\begin{array}{l}\text { Maximum } \\
\text { volume } \\
\text { (L) }\end{array}$ \\
\hline$D L=5$ & 8.9 & 32 & 3900 & $T F-2$ & 13.5 & 33 & 5300 \\
\hline$D L-11$ & 14.7 & 39 & 6000 & $T F-4$ & 9.8 & 43 & 6400 \\
\hline \multirow[t]{2}{*}{$D L=14 A$} & 8.7 & 26 & 3800 & $T F-7$ & 9.3 & 18 & 1700 \\
\hline & & & & $T F-11$ & 6.3 & 28 & 2500 \\
\hline MF -5 & 9.1 & 45 & 2600 & $T F-14$ & 9.7 & 29 & 2700 \\
\hline$M F-6$ & 14.9 & 44 & 4900 & & & & \\
\hline$M F-8$ & 8.6 & 22 & 2400 & CAT- 1 & $\cdots$ & -- & 8900 \\
\hline \multirow[t]{2}{*}{ MF - 9} & 15.6 & 53 & 8200 & CAT -2 & $\cdots$ & -- & 61 \\
\hline & & & & CAT -3 & $\cdots$ & $\cdots$ & 38 \\
\hline$M V-5$ & 2.7 & 24 & 640 & CAT -4 & $\cdots$ & $\cdots$ & 35 \\
\hline MV- 11 & 12.3 & 35 & 5300 & CAT -5 & -. & - & 120 \\
\hline$M V-13$ & 7.4 & 32 & 2200 & LAKE - 1 & $\cdots$ & $-\cdot$ & 230 \\
\hline$M V-14 A$ & 2.5 & 28 & 690 & GRAPE - 1 & $\cdots$ & - & 110 \\
\hline$M V-14 B$ & 14.5 & 28 & 3800 & GRAPE - 2 & $\cdots$ & - & 91 \\
\hline$M V-25$ & 11.4 & 32 & 3300 & & & & \\
\hline \multirow[t]{2}{*}{$M V-35$} & 5.3 & 0 & 2400 & HO-1 & $\cdots$ & $\cdots$ & 3000 \\
\hline & & & & HO-2 & $\cdots$ & $\cdots$ & 440 \\
\hline SF-2 & 5.1 & 25 & 1300 & $\mathrm{HO}-3$ & $\cdots$ & $\cdots$ & 620 \\
\hline$S F-8$ & 6.4 & 0 & 2100 & $80-1$ & $\cdots$ & $\cdots$ & 0 \\
\hline$S F-9 A$ & 6.7 & 30 & 2300 & $5.0-2$ & $\cdots$ & - & 300 \\
\hline$S F-10 B$ & 14.6 & 44 & 5100 & $B O-3$ & $\cdots$ & - & 98 \\
\hline$S F-12 B$ & 5.6 & 16 & 890 & BO- 4 & ... & -- & 310 \\
\hline$S F-13$ & 8.4 & 39 & 2700 & BO. 5 & $\cdots$ & $\cdots$ & 660 \\
\hline$S F-18$ & 10.7 & 34 & 2400 & & & & \\
\hline$S F-19$ & 12.0 & 29 & 3500 & & & & \\
\hline
\end{tabular}


Appendix B: Individual Constituent Concentrations in Pothole Water Samples 


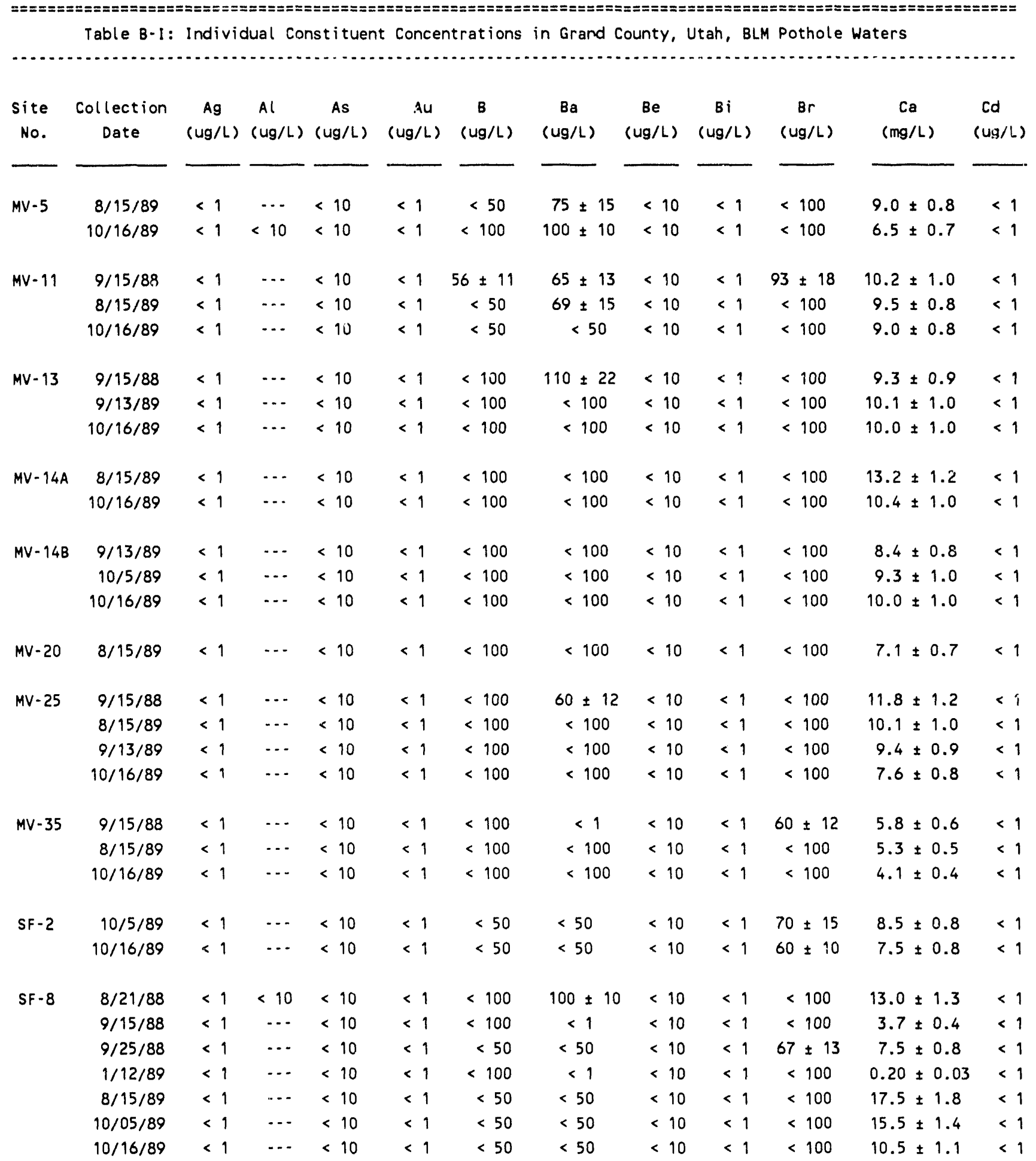


Table B-I: Individual Constituent Concentrations in Grand County, Utah, BLM Pothole Waters (cont.)

\begin{tabular}{|c|c|c|c|c|c|c|c|c|c|c|c|c|}
\hline $\begin{array}{l}\text { Site } \\
\text { No. }\end{array}$ & $\begin{array}{c}\text { Collection } \\
\text { Date }\end{array}$ & $\begin{array}{c}A g \\
(u g / L)\end{array}$ & $\begin{array}{c}A l \\
(u g / L)\end{array}$ & $\begin{array}{c}\text { As } \\
(u g / L)\end{array}$ & $\begin{array}{c}A u \\
(u g / L)\end{array}$ & $\begin{array}{c}\text { B } \\
(u g / L)\end{array}$ & $\begin{array}{c}B a \\
(u g / L)\end{array}$ & $\begin{array}{c}B e \\
(u g / L)\end{array}$ & $\begin{array}{c}B i \\
(u g / L)\end{array}$ & $\begin{array}{c}B r \\
(u g / L)\end{array}$ & $\begin{array}{c}\mathrm{Ca} \\
(\mathrm{mg} / \mathrm{L})\end{array}$ & $\begin{array}{c}\text { Cd } \\
(u g / L)\end{array}$ \\
\hline \multirow[t]{2}{*}{$S F-9 A$} & $10 / 05 / 89$ & $<1$ & $\cdots$ & $<10$ & $<1$ & $<50$ & $<50$ & $<10$ & $<1$ & $<100$ & $14.5 \pm 1.4$ & $<1$ \\
\hline & $10 / 16 / 89$ & $<1$ & $\cdots$ & $<10$ & $<1$ & $<50$ & $<50$ & $<10$ & $<1$ & $<100$ & $17.5 \pm 1.8$ & $<1$ \\
\hline$S F-9 B$ & $7 / 28 / 88$ & $<1$ & $<10$ & $<10$ & $<1$ & $8 \pm 1$ & $100 \pm 10$ & $<10$ & $<1$ & $<100$ & $\cdots$ & $<1$ \\
\hline \multirow[t]{8}{*}{$S F-10 B$} & $7 / 28 / 88$ & $<1$ & $<10$ & $<10$ & $<1$ & $10 \pm 1$ & $100 \pm 10$ & $<10$ & $<1$ & $<100$ & $\cdots$ & $<1$ \\
\hline & $8 / 21 / 88$ & $<1$ & $<10$ & $<10$ & $<1$ & $<100$ & $100 \pm 10$ & $<10$ & $<1$ & $<100$ & $30 \pm 3$ & $<1$ \\
\hline & $9 / 15 / 88$ & $<1$ & $\cdots$ & $<10$ & $<1$ & $57 \pm 12$ & $47 \pm 9$ & $<10$ & $<1$ & $89 \pm 18$ & $8.4 \pm 0.8$ & $<1$ \\
\hline & $9 / 25 / 88$ & $<1$ & $\cdots$ & $<10$ & $<1$ & $45 \pm 9$ & $73 \pm 14$ & $<10$ & $<1$ & $151 \pm 31$ & $21 \pm 2$ & $<1$ \\
\hline & $1 / 12 / 89$ & $<1$ & $\cdots$ & $<10$ & $<1$ & $<100$ & $<1$ & $<10$ & $<1$ & $<100$ & $0.37 \pm 0.04$ & $<1$ \\
\hline & $8 / 15 / 89$ & $<1$ & $<10$ & $<10$ & $<1$ & $<100$ & $100 \pm 10$ & $<10$ & $<1$ & $<100$ & $32 \pm 3$ & $<1$ \\
\hline & $10 / 05 / 89$ & $<1$ & $<10$ & $<10$ & $<1$ & $<100$ & $100 \pm 10$ & $<10$ & $<1$ & $<100$ & $20 \pm 3$ & $<1$ \\
\hline & $10 / 16 / 89$ & $<1$ & $<10$ & $<10$ & $<1$ & 100 & $120 \pm 10$ & $<10$ & $<1$ & $<100$ & $19 \pm 2$ & $<1$ \\
\hline \multirow[t]{2}{*}{$S F-12 A$} & $7 / 28 / 88$ & $<1$ & $<101$ & $10 \pm 1$ & $<1$ & $10 \pm 1$ & $200 \pm 20$ & $<10$ & $<1$ & $<100$ & $\cdots$ & $<1$ \\
\hline & $9 / 25 / 88$ & $<1$ & $\cdots$ & $<10$ & $<1$ & $48 \pm 10$ & $<50$ & $<10$ & $<1$ & $154 \pm 30$ & $20.8 \pm 2.1$ & $<1$ \\
\hline \multirow[t]{8}{*}{$S F-12 B$} & $7 / 28 / 88$ & $<1$ & $<10$ & $<10$ & $<1$ & $10 \pm 1$ & $500 \pm 50$ & $<10$ & $<1$ & $<100$ & $\cdots$ & $<1$ \\
\hline & $8 / 21 / 88$ & $<1$ & $<10$ & $<10$ & $<1$ & $<100$ & $100 \pm 10$ & $<10$ & $<1$ & $<100$ & $33 \pm 3$ & $<1$ \\
\hline & $9 / 15 / 88$ & -1 & $\cdots$ & $<10$ & $<1$ & $54 \pm 10$ & $47 \leq 9$ & $<10$ & $<1$ & $90 \pm 18$ & $9.5 \pm 1.0$ & $<1$ \\
\hline & $9 / 25 / 88$ & $<1$ & -- & $<10$ & $<1$ & $<50$ & $<50$ & $<10$ & $<1$ & $260 \pm 52$ & $22 \pm 2$ & $<1$ \\
\hline & $1 / 12 / 89$ & $<1$ & $\cdots$ & $<10$ & $<1$ & $<100$ & $<1$ & $<10$ & $<1$ & $<100$ & $<0.03$ & $<1$ \\
\hline & $8 / 15 / 89$ & $<1$ & $<10$ & $<10$ & $<1$ & $<100$ & $100 \pm 10$ & $<10$ & $<1$ & $<100$ & $31 \pm 3$ & $<1$ \\
\hline & $10 / 05 / 89$ & $<1$ & $<10$ & $<10$ & $<1$ & $<100$ & $<100$ & $<10$ & $<1$ & $<100$ & $27 \pm 3$ & $<1$ \\
\hline & $10 / 16 / 89$ & $<1$ & $<10$ & $<10$ & $<1$ & $<100$ & $<100$ & $<10$ & $<1$ & $<100$ & $24 \pm 2$ & $<1$ \\
\hline \multirow[t]{7}{*}{$S F-13$} & $7 / 28 / 88$ & $<1$ & $<10$ & $<10$ & $<1$ & $10 \pm 1$ & $100 \pm 10$ & $<10$ & $<1$ & $<100$ & $\ldots$ & $<1$ \\
\hline & $8 / 21 / 88$ & $<1$ & $<10$ & $<10$ & $<1$ & $<100$ & $100 \pm 10$ & $<10$ & $<1$ & $<100$ & $29 \pm 3$ & $<1$ \\
\hline & $9 / 15 / 88$ & $<1$ & $\cdots$ & $<10$ & $<1$ & $55 \pm 11$ & $59 \pm 12$ & $<10$ & $<1$ & $77 \pm 15$ & $9.6 \pm 1.0$ & $<1$ \\
\hline & $1 / 12 / 89$ & $<1$ & $\cdots$ & $<10$ & $<1$ & $<100$ & $<1$ & $<10$ & $<1$ & $<100$ & $0.27 \pm 0.03$ & $<1$ \\
\hline & $8 / 15 / 89$ & $<1$ & $<10$ & $<10$ & $<1$ & $<100$ & $100 \pm 10$ & $<10$ & $<1$ & $<100$ & $34 \pm 3$ & $<1$ \\
\hline & $10 / 05 / 89$ & $<1$ & $<10$ & $<10$ & $<1$ & $<100$ & $100 \pm 10$ & $<10$ & $<1$ & $<100$ & $19 \pm 2$ & $<1$ \\
\hline & $10 / 16 / 89$ & $<1$ & $<10$ & $<10$ & $<1$ & $<100$ & $100 \pm 10$ & $<10$ & $<1$ & $<100$ & $14 \pm 2$ & $<1$ \\
\hline \multirow[t]{2}{*}{$S F-18$} & $10 / 05 / 89$ & $<1$ & $<10$ & $<10$ & $<1$ & $<100$ & $<100$ & $<10$ & $<1$ & $<100$ & $19 \pm 3$ & $<1$ \\
\hline & $10 / 16 / 89$ & $<1$ & $<10$ & $<10$ & $<1$ & $<100$ & $<100$ & $<10$ & $<1$ & $<100$ & $16 \pm 2$ & $<1$ \\
\hline \multirow[t]{6}{*}{$S F-19$} & $8 / 21 / 88$ & $<1$ & $<10$ & $<10$ & $<1$ & $<100$ & $100 \pm 10$ & $<10$ & $<1$ & $<100$ & $27 \pm 3$ & $<1$ \\
\hline & $9 / 15 / 88$ & $<1$ & $\cdots$ & $<10$ & $<1$ & $<100$ & $<1$ & $<10$ & $<1$ & $61 \pm 12$ & $6.7 \pm 0.7$ & $<1$ \\
\hline & $9 / 25 / 88$ & $<1$ & $\cdots$ & $<10$ & $<1$ & $<50$ & $<50$ & $<10$ & $<1$ & $80 \pm 16$ & $13.8 \pm 1.4$ & $<1$ \\
\hline & $1 / 12 / 89$ & $<1$ & $\cdots$ & $<10$ & $<1$ & $<100$ & $<1$ & $<10$ & $<1$ & $<100$ & $0.34 \pm 0.03$ & $<1$ \\
\hline & $8 / 15 / 89$ & $<1$ & $<10$ & $<10$ & $<1$ & $<100$ & $100 \pm 10$ & $<10$ & $<1$ & $<100$ & $25 \pm 3$ & $<1$ \\
\hline & $0 / 16 / 89$ & $<1$ & $<10$ & $<10$ & $<1$ & $<100$ & $<100$ & $<10$ & $<1$ & $<100$ & $11 \pm 2$ & $<1$ \\
\hline
\end{tabular}




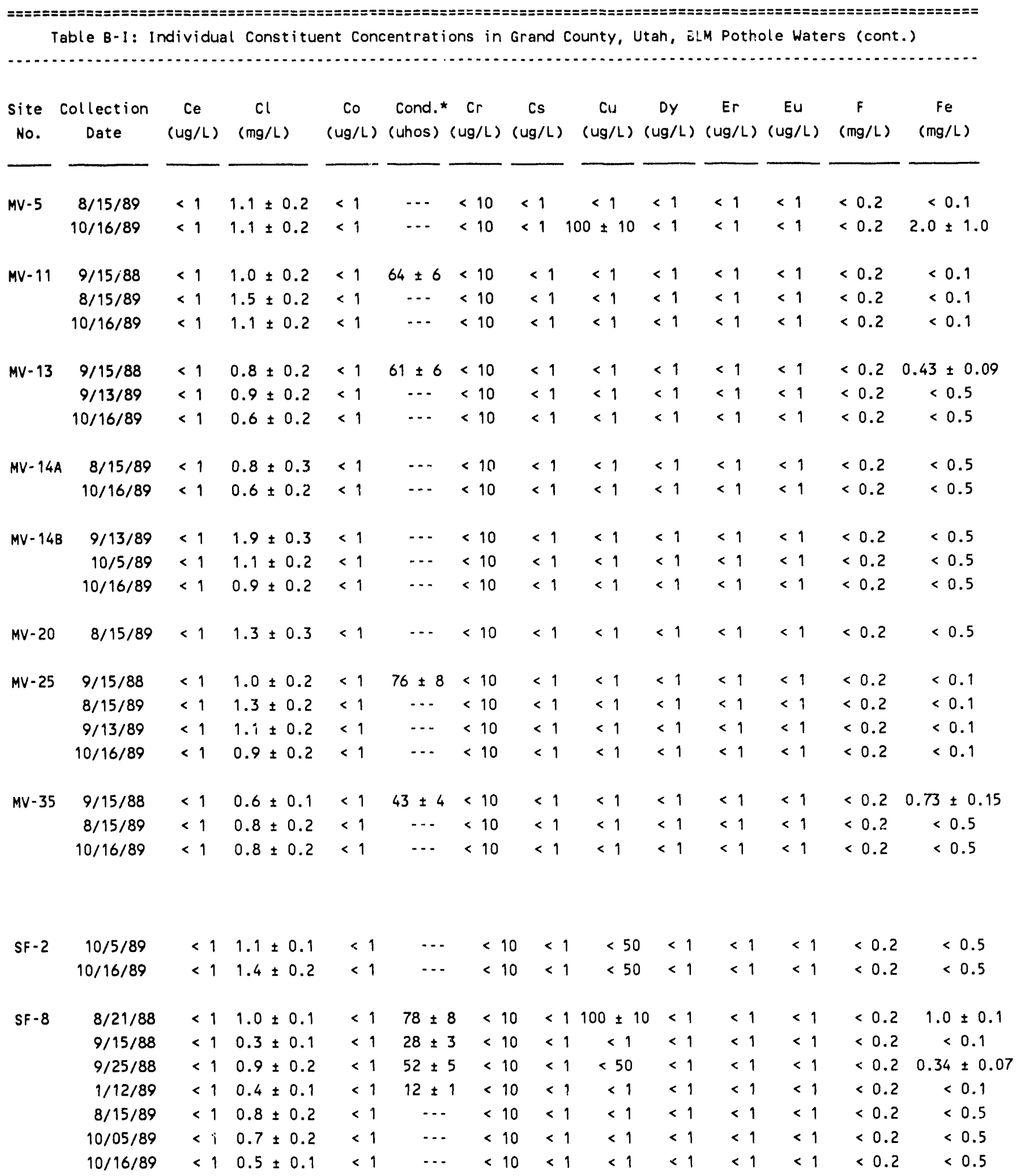

* Cond. = Conductivity 


\begin{tabular}{|c|c|c|c|c|c|c|c|c|c|c|c|c|c|}
\hline $\begin{array}{l}\text { Site } \\
\text { No. }\end{array}$ & $\begin{array}{c}\text { Collection } \\
\text { Date }\end{array}$ & $\begin{array}{c}\mathrm{Ce} \\
(u g / L)\end{array}$ & $\begin{array}{c}\mathrm{Cl} \\
(\mathrm{mg} / \mathrm{L})\end{array}$ & $\begin{array}{c}\text { Co } \\
\text { (ug/L) }\end{array}$ & $\begin{array}{l}\text { Cond.* } \\
\text { (uhos) (u }\end{array}$ & $\begin{array}{l}\mathrm{Cr} \\
\mathrm{ug} / \mathrm{L})(\mathrm{L}\end{array}$ & $\begin{array}{c}\text { Cs } \\
(u g / L)\end{array}$ & $\begin{array}{c}\mathrm{Cu} \\
(\mathrm{ug} / \mathrm{L})\end{array}$ & $\begin{array}{c}\text { Dy } \\
(u g / L)\end{array}$ & $\begin{array}{c}E r \\
(u g / L)\end{array}$ & $\begin{array}{c}\mathrm{Eu} \\
(\mathrm{ug} / \mathrm{L})\end{array}$ & $\begin{array}{c}F \\
(\mathrm{mg} / \mathrm{L})\end{array}$ & $\begin{array}{c}\mathrm{Fe} \\
(\mathrm{mg} / \mathrm{L})\end{array}$ \\
\hline \multirow[t]{2}{*}{$S F-9 A$} & $10 / 05 / 89$ & $<1$ & $1.2 \pm 0.2$ & $<1$ & $\cdots$ & $<10$ & $<1$ & $<1$ & $<1$ & $<1$ & $<1$ & $<0.2$ & $<0.5$ \\
\hline & $10 / 16 / 89$ & $<1$ & $0.8 \pm 0.2$ & $<1$ & $\cdots$ & $<10$ & $<1$ & $<1$ & $<1$ & $<1$ & $<1$ & $<0.2$ & $<0.5$ \\
\hline$S F-9 B$ & $7 / 28 / 88$ & $10 \pm 1$ & $2.4 \pm 0.2$ & $<1$ & $168 \pm 17$ & $10 \pm 1$ & $1<1$ & $10 \pm 1$ & $<1$ & $<1$ & $<1$ & $0.4 \pm 0.1$ & $1.0 \pm 0.1$ \\
\hline \multirow[t]{8}{*}{$S F-10 B$} & $7 / 28 / 88$ & $<1$ & $4.2 \pm 0.4$ & $<1$ & $245 \pm 24$ & $10 \pm 1$ & $1<1$ & $10 \pm 1$ & $<1$ & $<1$ & $<1$ & $0.5 \pm 0.1$ & $1.0 \pm 0.1$ \\
\hline & $8 / 21 / 88$ & $<1$ & $2.8 \pm 0.3$ & $<1$ & $171 \pm 17$ & $<10$ & $<1$ & $100 \pm 10$ & $<1$ & $<1$ & $<1$ & $0.3 \pm 0.1$ & $1.0 \pm 0.1$ \\
\hline & $9 / 15 / 88$ & $<1$ & $1.1 \pm 0.1$ & $<1$ & $51 \pm 5$ & $<10$ & $<1$ & $<1$ & $<1$ & $<1$ & $<1$ & $<0.2$ & $<0.1$ \\
\hline & $9 / 25 / 88$ & $<1$ & $2.2 \pm 0.2$ & $<1$ & $129 \pm 13$ & $<10$ & $<1$ & $<50$ & $<1$ & $<1$ & $<1$ & $<0.2$ & $0.48 \pm 0.10$ \\
\hline & $1 / 12 / 89$ & $<1$ & $0.4 \pm 0.1$ & $<1$ & $3.5 \pm 0.4$ & $<10$ & $<1$ & $<1$ & $<1$ & $<1$ & $<1$ & $0.3 \pm 0.2$ & $<0.1$ \\
\hline & $8 / 15 / 89$ & $<1$ & $3.2 \pm 0.4$ & $<1$ & $\cdots$ & $<10$ & $<1$ & $<50$ & $<1$ & $<1$ & $<1$ & $0.5 \pm 0.1$ & $0.7 \pm 0.2$ \\
\hline & $10 / 05 / 89$ & $<1$ & $2.2 \pm 0.3$ & $<1$ & $\cdots$ & $<10$ & $<9$ & $<50$ & $<1$ & $<1$ & $<1$ & $0.4 \pm 0.1$ & $0.6 \pm 0.3$ \\
\hline & $10 / 16 / 89$ & $<1$ & $1.9 \pm 0.2$ & $<1$ & $\cdots$ & $<10$ & $<1$ & $<50$ & $<1$ & $<1$ & $<1$ & $0.5 \pm 0.1$ & $<0.5$ \\
\hline \multirow[t]{2}{*}{$S F-12 A$} & $7 / 28 / 88$ & $<1$ & $6.7 \pm 0.7$ & $<1$ & $310 \pm 31$ & $10 \pm 1$ & $1<1$ & $<1$ & $<1$ & $<1$ & $<1$ & $0.7 \pm 0.1$ & $1.0 \pm 0.1$ \\
\hline & $9 / 25 / 88$ & $<1$ & $2.0 \pm 0.2$ & $<1$ & $125 \pm 13$ & $<10$ & $<1$ & $<50$ & $<1$ & $<1$ & $<1$ & $0.2 \pm 0.1$ & $<0.1$ \\
\hline \multirow[t]{8}{*}{$S F-12 B$} & $7 / 28 / 88$ & $<1$ & $2.8 \pm 0.3$ & $<1$ & $210 \pm 21$ & $10 \pm 1$ & $1<1$ & $10 \pm 1$ & $<1$ & $<1$ & $<1$ & $0.6 \pm 0.1$ & $1.0 \pm 0.1$ \\
\hline & $8 / 21 / 88$ & $<1$ & $2.9 \pm 0.3$ & $<1$ & $194 \pm 19$ & $<10$ & $<1$ & $100 \pm 10$ & $<1$ & $<1$ & $<1$ & $0.3 \pm 0.1$ & $5.0 \pm 0.5$ \\
\hline & $9 / 15 / 88$ & $<1$ & $1.0 \pm 0.1$ & $<1$ & $64 \pm 6$ & $<10$ & $<1$ & $<1$ & $<1$ & $<1$ & $<1$ & $<0.2$ & $<0.1$ \\
\hline & $9 / 25 / 88$ & $<1$ & $3.1 \pm 0.3$ & $<1$ & $134 \pm 13$ & $<10$ & $<1$ & $<50$ & $<1$ & $<1$ & $<1$ & $0.3 \pm 0.1$ & $<0.1$ \\
\hline & $1 / 12 / 89$ & $<1$ & $0.4 \pm 0.1$ & $<1$ & $15 \pm 2$ & $<10$ & $<1$ & $<1$ & $<1$ & $<1$ & $<1$ & $<0.2$ & $<0.1$ \\
\hline & $8 / 15 / 89$ & $<1$ & $2.5 \pm 0.3$ & $<1$ & $\cdots$ & $<10$ & $<1$ & $<50$ & $<1$ & $<1$ & $<1$ & $0.3 \pm 0.1$ & $3.9 \pm 0.4$ \\
\hline & $10 / 05 / 89$ & $<1$ & $2.2 \pm 0.3$ & $<1$ & $\cdots$ & $<10$ & $<1$ & $<50$ & $<1$ & $<1$ & $<1$ & $0.3 \pm 0.1$ & $3.4 \pm 0.4$ \\
\hline & $10 / 16 / 89$ & $<1$ & $2.0 \pm 0.3$ & $<1$ & $\cdots$ & $<10$ & $<1$ & $<50$ & $<1$ & $<1$ & $<1$ & $0.3 \pm 0.1$ & $2.9 \pm 0.3$ \\
\hline \multirow[t]{7}{*}{$S F-13$} & $7 / 28 / 88$ & $<1$ & $2.7 \pm 0.3$ & $=1$ & $192 \pm 19$ & $10 \pm 1$ & $1<1$ & $<1$ & $<1$ & $<1$ & $<1$ & $0.5 \pm 0.1$ & $1.0 \pm 0.1$ \\
\hline & $8 / 21 / 88$ & $<1$ & $2.4 \pm 0.2$ & $<1$ & $167 \pm 17$ & $<10$ & $<1$ & $100 \pm 10$ & $<1$ & $<1$ & $<1$ & $0.3 \pm 0.1$ & $5.0 \pm 0.5$ \\
\hline & $9 / 15 / 88$ & $<1$ & $0.5 \pm 0.1$ & $<1$ & $60 \pm 6$ & $<10$ & $<1$ & $<1$ & $<1$ & $<1$ & $<1$ & $<0.2$ & $0.36 \pm 0.07$ \\
\hline & $1 / 12 / 89$ & $<1$ & $2.9 \pm 0.3$ & $<1$ & $20 \pm 2$ & $<10$ & $<1$ & $<1$ & $<1$ & $<1$ & $<1$ & $<0.2$ & $<0.1$ \\
\hline & $8 / 15 / 89$ & $<1$ & $2.5 \pm 0.3$ & $<1$ & $\cdots$ & $10 \pm 1$ & $1<1$ & $<1$ & $<1$ & $<1$ & $<1$ & $0.5 \pm 0.1$ & $1.4 \pm 0.1$ \\
\hline & $10 / 05 / 89$ & $<1$ & $2.4 \pm 0.3$ & $<1$ & $\cdots$ & $<10$ & $<1$ & $<1$ & $<1$ & $<1$ & $<1$ & $0.5 \pm 0.1$ & $1.3 \pm 0 . i$ \\
\hline & $10 / 16 / 89$ & $<1$ & $1.8 \pm 0.2$ & $<1$ & $\cdots$ & $<10$ & $<1$ & $<1$ & $<1$ & $<1$ & $<1$ & $0.5 \pm 0.1$ & $1.1 \pm 0.2$ \\
\hline \multirow[t]{2}{*}{$S F-18$} & $10 / 05 / 89$ & $<1$ & $0.5 \pm 0.1$ & $<1$ & - . & $<10$ & $<1$ & $<1$ & $<1$ & $<1$ & $<1$ & $<0.2$ & $<0.5$ \\
\hline & $10 / 16 / 89$ & $<1$ & $0.4 \pm 0.2$ & $<1$ & $\cdots$ & $<10$ & $<1$ & $<1$ & $<1$ & $<1$ & $<1$ & $<0.2$ & $<0.5$ \\
\hline \multirow[t]{6}{*}{$S F-19$} & $8 / 21 / 88$ & $<1$ & $1.3 \pm 0.2$ & $<1$ & $151 \pm 15$ & $<10$ & $<1$ & $100 \pm 10$ & $<1$ & $<1$ & $<1$ & $0.2 \pm 0.1$ & $5.0 \pm 0.5$ \\
\hline & $9 / 15 / 88$ & $<1$ & $0.4 \pm 0.1$ & $<1$ & $41 \pm 4$ & $<10$ & $<1$ & $<1$ & $<1$ & $<1$ & $<1$ & $<0.2$ & $<0.1$ \\
\hline & $9 / 25 / 88$ & $<1$ & $1.0 \pm 0.2$ & $<1$ & $75 \pm 8$ & $<10$ & $<1$ & $<50$ & $<1$ & $<1$ & $<1$ & $0.2 \pm 0.1$ & $<0.1$ \\
\hline & $1 / 12 / 89$ & $<1$ & $<0.2$ & $<1$ & $5.0 \pm 0.5$ & $;<10$ & $<1$ & $<1$ & $<1$ & $<1$ & $<1$ & $<0.2$ & $<0.1$ \\
\hline & $8 / 15 / 89$ & $<1$ & $1.5 \pm 0.2$ & $<1$ & $\cdots$ & $<10$ & $<1$ & $<1$ & $<1$ & $<1$ & $<1$ & $<0.2$ & $<0.5$ \\
\hline & $10 / 16 / 89$ & $<1$ & $0.8 \pm 0.1$ & $<1$ & $\cdots$ & $<10$ & $<1$ & $<1$ & $<1$ & $<1$ & $<1$ & $<0.2$ & $<0.5$ \\
\hline
\end{tabular}




\begin{tabular}{|c|c|c|c|c|c|c|c|c|c|c|c|c|c|}
\hline $\begin{array}{l}\text { Site } \\
\text { No. }\end{array}$ & $\begin{array}{c}\text { Collection } \\
\text { Date }\end{array}$ & $\begin{array}{c}G a \\
(u g / L)\end{array}$ & $\begin{array}{c}\text { Gd } \\
(u g / L)\end{array}$ & $\begin{array}{c}\mathrm{Ge} \\
(\mathrm{ug} / \mathrm{L})\end{array}$ & $\begin{array}{c}H f \\
(u g / L)\end{array}$ & $\begin{array}{c}\mathrm{Hg} \\
(\mathrm{ug} / \mathrm{L})\end{array}$ & $\begin{array}{c}\text { Ho } \\
(u g / L)\end{array}$ & $\begin{array}{c}\text { I } \\
(u g / L)\end{array}$ & $\begin{array}{c}\ln \\
(u g / L)\end{array}$ & $\begin{array}{c}\text { Ir } \\
(u g / L)\end{array}$ & $\begin{array}{c}K \\
(m g / L)\end{array}$ & $\begin{array}{c}\text { La } \\
(\mathrm{ug} / \mathrm{L})\end{array}$ & $\begin{array}{c}L i \\
(u g / L)\end{array}$ \\
\hline \multirow[t]{2}{*}{$M V-5$} & $8 / 15 / 89$ & $<1$ & $<1$ & $<1$ & $<1$ & $<1$ & $<1$ & $<1$ & $<1$ & $<1$ & $1.2 \pm 0.1$ & $<1$ & $<10$ \\
\hline & $10 / 16 / 89$ & $<1$ & $<1$ & $<1$ & $<1$ & $<1$ & $<1$ & $<10$ & $<1$ & $<1$ & $1.8 \pm 0.2$ & $<1$ & $<10$ \\
\hline \multirow[t]{3}{*}{ MV- 11} & $9 / 15 / 88$ & $<1$ & $<1$ & $<1$ & $<1$ & $<1$ & $<1$ & $<1$ & $<1$ & $<1$ & $1.0 \pm 0.1$ & $<1$ & $<10$ \\
\hline & $8 / 15 / 89$ & $<1$ & $<1$ & $<1$ & $<1$ & $<1$ & $<1$ & $<1$ & $<1$ & $<1$ & $1.1 \pm 0.1$ & $<1$ & $<10$ \\
\hline & $10 / 16 / 89$ & $<1$ & $<1$ & $<1$ & $<1$ & $<1$ & $<1$ & $<1$ & $<1$ & $<1$ & $1.2 \pm 0.1$ & $<1$ & $<10$ \\
\hline \multirow[t]{3}{*}{$M V-13$} & $9 / 15 / 88$ & $<1$ & $<1$ & $<1$ & $<1$ & $<1$ & $<1$ & $<1$ & $<1$ & $<1$ & $0.8 \pm 0.1$ & $<1$ & $<10$ \\
\hline & $9 / 13 / 89$ & $<1$ & $<1$ & $<1$ & $<1$ & $<1$ & $<1$ & $<1$ & $<1$ & $<1$ & $1.1 \pm 0.1$ & $<1$ & $<10$ \\
\hline & $10 / 16 / 89$ & $<1$ & $<1$ & $<1$ & $<1$ & $<1$ & $<1$ & $<1$ & $<1$ & $<1$ & $1.0 \pm 0.1$ & $<1$ & $<10$ \\
\hline \multirow{2}{*}{$M V-14 A$} & $8 / 15 / 89$ & $<1$ & $<1$ & $<1$ & $<1$ & $<1$ & $<1$ & $<1$ & $<1$ & $<1$ & $1.4 \pm 0.1$ & $<1$ & $<10$ \\
\hline & $10 / 16 / 89$ & $<1$ & $<1$ & $<1$ & $<1$ & $<1$ & $<1$ & $<1$ & $<1$ & $<1$ & $1.1 \pm 0.1$ & $<1$ & $<10$ \\
\hline \multirow{3}{*}{$M V-14 B$} & $9 / 13 / 89$ & $<1$ & $<1$ & $<1$ & $<1$ & $<1$ & $<1$ & $<1$ & $<1$ & $<1$ & $1.2 \pm 0.1$ & $<1$ & $<10$ \\
\hline & $10 / 5 / 89$ & $<1$ & $<1$ & $<1$ & $<1$ & $<1$ & $<1$ & $<1$ & $<1$ & $<1$ & $1.2 \pm 0.1$ & $<1$ & $<10$ \\
\hline & $10 / 16 / 89$ & $<1$ & $<1$ & $<1$ & $<1$ & $<1$ & $<1$ & $<1$ & $<1$ & $<1$ & $1.2 \pm 0.1$ & $<1$ & $<10$ \\
\hline$M V-20$ & $8 / 15 / 89$ & $<1$ & $<1$ & $<1$ & $<1$ & $<1$ & $<1$ & $<1$ & $<1$ & $<1$ & $1.6 \pm 0.2$ & $<1$ & $<10$ \\
\hline \multirow[t]{4}{*}{$M V-25$} & $9 / 15 / 88$ & $<1$ & $<1$ & $<1$ & $<1$ & $<1$ & $<1$ & $<1$ & $<1$ & $<1$ & $1.1 \pm 0.1$ & $<1$ & $<10$ \\
\hline & $8 / 15 / 89$ & $<1$ & $<1$ & $<1$ & $<1$ & $<1$ & $<1$ & $<1$ & $<1$ & $<1$ & $1.3 \pm 0.1$ & $<1$ & $<10$ \\
\hline & $9 / 13 / 89$ & $<1$ & $<1$ & $<1$ & $<1$ & $<1$ & $<1$ & $<1$ & $<1$ & $<1$ & $1.2 \pm 0.1$ & $<1$ & $<10$ \\
\hline & $10 / 16 / 89$ & $<1$ & $<1$ & $<1$ & $<1$ & $<1$ & $<1$ & $<1$ & $<1$ & $<1$ & $1.4 \pm 0.1$ & $<1$ & $<10$ \\
\hline \multirow[t]{3}{*}{$M V-35$} & $9 / 15 / 88$ & $<1$ & $<1$ & $<1$ & $<1$ & $<1$ & $<1$ & $<1$ & $<1$ & $<1$ & $0.9 \pm 0.1$ & $<1$ & $<10$ \\
\hline & $8 / 15 / 89$ & $<1$ & $<1$ & $<1$ & $<1$ & $<1$ & $<1$ & $<1$ & $<1$ & $<1$ & $1.1 \pm 0.1$ & $<1$ & $<10$ \\
\hline & $10 / 16 / 89$ & $<1$ & $<1$ & $<1$ & $<1$ & $<1$ & $<1$ & $<1$ & $<1$ & $<1$ & $1.0 \pm 0.1$ & $<1$ & $<10$ \\
\hline \multirow[t]{2}{*}{$S F-2$} & $10 / 5 / 89$ & $<1$ & $<1$ & $<1$ & $<1$ & $<1$ & $<1$ & $<1$ & $<1$ & $<1$ & $1.0 \pm 0.1$ & $<1$ & $<10$ \\
\hline & $10 / 16 / 89$ & $<1$ & $<1$ & $<1$ & $<1$ & $<1$ & $<1$ & $<1$ & $<1$ & $<1$ & $1.2 \pm 0.1$ & $<1$ & $<10$ \\
\hline \multirow[t]{7}{*}{$S F-8$} & $8 / 21 / 88$ & $<1$ & $<1$ & $<1$ & $<1$ & $<1$ & $<1$ & $10 \pm 1$ & $<1$ & $<1$ & $0.98 \pm 0.10$ & $<1$ & $<10$ \\
\hline & $9 / 15 / 88$ & $<1$ & $<1$ & $<1$ & $<1$ & $<1$ & $<1$ & $<1$ & $<1$ & $<1$ & $0.4 \pm 0.1$ & $<1$ & $<10$ \\
\hline & $9 / 25 / 88$ & $<1$ & $<1$ & $<1$ & $<1$ & $<1$ & $<1$ & $<1$ & $<1$ & $<1$ & $0.6 \pm 0.1$ & $<1$ & $<10$ \\
\hline & $1 / 12 / 89$ & $<1$ & $<1$ & $<1$ & $<1$ & $<1$ & $<1$ & $<1$ & $<1$ & $<1$ & $0.36 \pm 0.09$ & $<1$ & $<10$ \\
\hline & $8 / 15 / 89$ & $<1$ & $<1$ & $<1$ & $<1$ & $<1$ & $<1$ & $<1$ & $<1$ & $<1$ & $0.6 \pm 0.1$ & $<1$ & $<10$ \\
\hline & $10 / 05 / 89$ & $<1$ & $<1$ & $<1$ & $<1$ & $<1$ & $<1$ & $<1$ & $<1$ & $<1$ & $0.6 \pm 0.1$ & $<1$ & $<10$ \\
\hline & $10 / 16 / 89$ & $<1$ & $<1$ & $<1$ & $<1$ & $<1$ & $<1$ & $<1$ & $<1$ & $<1$ & $0.5 \pm 0.1$ & $<1$ & $<10$ \\
\hline
\end{tabular}




\begin{tabular}{|c|c|c|c|c|c|c|c|c|c|c|c|c|c|}
\hline $\begin{array}{l}\text { Site } \\
\text { No. }\end{array}$ & $\begin{array}{c}\text { Collection } \\
\text { Date }\end{array}$ & $\begin{array}{c}G a \\
(u g / L)\end{array}$ & $\begin{array}{c}G d \\
(u g / L)\end{array}$ & $\begin{array}{c}\mathrm{Ge} \\
(\mathrm{ug} / \mathrm{L})\end{array}$ & $\begin{array}{c}H f \\
(u g / L)\end{array}$ & $\begin{array}{c}\mathrm{Hg} \\
(u g / L)\end{array}$ & $\begin{array}{c}\text { Ho } \\
(u g / L)\end{array}$ & $\begin{array}{c}I \\
(u g / L)\end{array}$ & $\begin{array}{c}\text { In } \\
(u g / L)\end{array}$ & $\begin{array}{c}\text { Ir } \\
(u g / L)\end{array}$ & $\begin{array}{c}K \\
(m g / L)\end{array}$ & $\begin{array}{c}\mathrm{La} \\
(\mathrm{ug} / \mathrm{L})\end{array}$ & $\begin{array}{c}\mathrm{Li} \\
(u g / L)\end{array}$ \\
\hline \multirow[t]{2}{*}{$S F-9 A$} & $10 / 05 / 89$ & $<1$ & $<1$ & $<1$ & $<1$ & $<1$ & $<1$ & $<1$ & $<1$ & $<1$ & $1.2 \pm 0.1$ & $<1$ & $<10$ \\
\hline & $10 / 16 / 89$ & $<1$ & $<1$ & $<1$ & $<1$ & $<1$ & $<1$ & $<1$ & $<1$ & $<1$ & $1.1 \pm 0.1$ & $<1$ & $<10$ \\
\hline$S F-9 B$ & $7 / 28 / 88$ & $<1$ & $<1$ & $<1$ & $<1$ & $<1$ & $<1$ & $10 \pm 1$ & $<1$ & $<1$ & $\cdots$ & $<1$ & $<10$ \\
\hline \multirow[t]{8}{*}{$S F-10 B$} & $7 / 28 / 88$ & $<1$ & $<1$ & $<1$ & $<1$ & $<1$ & $<1$ & $10 \pm 1$ & $<1$ & $<1$ & $\cdots$ & $<1$ & $<10$ \\
\hline & $8 / 21 / 88$ & $<1$ & $<1$ & $<1$ & $<1$ & $<1$ & $<1$ & $10 \pm 1$ & $<1$ & $<1$ & $2.4 \pm 0.2$ & $<1$ & $<10$ \\
\hline & $9 / 15 / 88$ & $<1$ & $<1$ & $<1$ & $: 1$ & $<1$ & $<1$ & $<1$ & $<1$ & $<1$ & $0.8 \pm 0.1$ & $<1$ & $<10$ \\
\hline & $9 / 25 / 88$ & $<1$ & $<1$ & $<1$ & $<1$ & $<1$ & $<1$ & $<1$ & $<1$ & $<1$ & $1.4 \pm 0.1$ & $<1$ & $<10$ \\
\hline & $1 / 12 / 89$ & $<1$ & $<1$ & $<1$ & $<1$ & $<1$ & $<1$ & $<1$ & $<1$ & $<1$ & $0.20 \pm 0.09$ & $<1$ & $<10$ \\
\hline & $8 / 15 / 89$ & $<1$ & $<1$ & $<1$ & $<1$ & $<1$ & $<1$ & $<1$ & $<1$ & $<1$ & $0.9 \pm 0.1$ & $<1$ & $<10$ \\
\hline & $10 / 05 / 89$ & $<1$ & $<1$ & $<1$ & $<1$ & $<1$ & $<1$ & $<1$ & $<1$ & $<1$ & $0.8 \pm 0.1$ & $<1$ & $<10$ \\
\hline & $10 / 16 / 89$ & $<1$ & $<1$ & $<1$ & $<1$ & $<1$ & $<1$ & $<1$ & $<1$ & $<1$ & $0.5 \pm 0.2$ & $<1$ & $<10$ \\
\hline \multirow[t]{2}{*}{$S F-12 A$} & $7 / 28 / 88$ & $<1$ & $<1$ & $<1$ & $<1$ & $<1$ & $<1$ & $10 \pm 1$ & $<1$ & $<i$ & $\cdots$ & $<1$ & $<10$ \\
\hline & $9 / 25 / 88$ & $<1$ & $<1$ & $<1$ & $<1$ & $<1$ & $<1$ & $<1$ & $<1$ & $<1$ & $1.5 \pm 0.2$ & $<1$ & $<10$ \\
\hline \multirow[t]{8}{*}{$S F-12 B$} & $7 / 28 / 88$ & $<1$ & $<1$ & $<1$ & $<1$ & $<1$ & $<1$ & $10 \pm 1$ & $<1$ & $<1$ & $\cdots$ & $<1$ & $<10$ \\
\hline & $8 / 21 / 88$ & $<1$ & $<1$ & $<1$ & $<1$ & $<1$ & $<1$ & $10 \pm 1$ & $<1$ & $<1$ & $2.8 \pm 0.3$ & $<1$ & $<10$ \\
\hline & $9 / 15 / 88$ & $<1$ & $<1$ & $<1$ & $<1$ & $<1$ & $<1$ & $<1$ & $<1$ & $<1$ & $0.8 \pm 0.1$ & $<1$ & $<10$ \\
\hline & $9 / 25 / 88$ & $<1$ & $<1$ & $<1$ & $<1$ & $<1$ & $<1$ & $25 \pm 5$ & $<1$ & $<1$ & $1.5 \pm 0.2$ & $<1$ & $<10$ \\
\hline & $1 / 12 / 89$ & $<1$ & $<1$ & $<1$ & $<1$ & $<1$ & $<1$ & $<1$ & $<1$ & $<1$ & $<0.09$ & $<1$ & $<10$ \\
\hline & $8,15 / 89$ & $<1$ & $<1$ & $<1$ & $<1$ & $\because 1$ & $<1$ & $<1$ & $<1$ & $<1$ & $0.8 \pm 0.1$ & $<1$ & $<10$ \\
\hline & $10 / 05 / 89$ & $<1$ & $<1$ & $<1$ & $<1$ & $<1$ & $<1$ & $<1$ & $<1$ & $<1$ & $0.9 \pm 0.1$ & $<1$ & $<10$ \\
\hline & $10 / 16 / 89$ & $<1$ & $<1$ & $<1$ & $<1$ & $<1$ & $<?$ & r i & $<1$ & $<1$ & $0.6 \pm 0.2$ & $<1$ & $<10$ \\
\hline \multirow[t]{7}{*}{$S F-13$} & $7 / 28 / 88$ & $<1$ & $<1$ & $<1$ & $<1$ & $<1$ & $<1$ & $10 \pm 1$ & $<1$ & $<1$ & $\cdots$ & $<1$ & $<10$ \\
\hline & $8 / 21 / 88$ & $<1$ & $<1$ & $<1$ & $<1$ & $<1$ & $<1$ & $10 \pm 1$ & $<1$ & $<1$ & $2.1 \pm 0.2$ & $<1$ & $<10$ \\
\hline & $9 / 15 / 88$ & $<1$ & $<1$ & $<1$ & $<1$ & $<1$ & $<1$ & $<1$ & $<1$ & $<1$ & $0.7 \pm 0.1$ & $<1$ & $<10$ \\
\hline & $1 / 12 / 89$ & $<1$ & $<1$ & $<1$ & $<1$ & $<1$ & $<1$ & $<1$ & $<1$ & $<1$ & $0.81 \pm 0.09$ & $<1$ & $<10$ \\
\hline & $8 / 15 / 89$ & $<1$ & $<1$ & $<1$ & $<1$ & $<1$ & $<1$ & $<1$ & $<1$ & $<1$ & $1.1 \pm 0.1$ & $<1$ & $<10$ \\
\hline & $10 / 05 / 89$ & $<1$ & $<1$ & $<1$ & $<1$ & $<1$ & $<1$ & $<1$ & $<1$ & $<1$ & $0.8 \pm 0.1$ & $<1$ & $<10$ \\
\hline & $10 / 16 / 89$ & $<1$ & $<1$ & $<1$ & $<1$ & $<1$ & $<1$ & $<1$ & $<1$ & $<1$ & $0.7 \pm 0.1$ & $<1$ & $<10$ \\
\hline \multirow[t]{2}{*}{ SF- 18} & $10 / 05 / 89$ & $<1$ & $<1$ & $<1$ & $<1$ & $<1$ & $<1$ & $<1$ & $<1$ & $<1$ & $1.0 \pm 0.1$ & $<1$ & $<10$ \\
\hline & $10 / 16 / 89$ & $<1$ & $<1$ & $<1$ & $<1$ & $<1$ & $<1$ & $<1$ & $<1$ & $<1$ & $0.9 \pm 0.1$ & $<1$ & $<10$ \\
\hline \multirow[t]{6}{*}{$S F-19$} & $8 / 21 / 88$ & $<1$ & $<1$ & $<1$ & $<1$ & $<1$ & $<1$ & $10 \pm 1$ & $<1$ & $<1$ & $2.1 \pm 0.2$ & $<1$ & $<10$ \\
\hline & $9 / 15 / 88$ & $<1$ & $<1$ & $<1$ & $<1$ & $<1$ & $<1$ & $<1$ & $<1$ & $<1$ & $0.6 \pm 0.1$ & $<1$ & $<10$ \\
\hline & $9 / 25 / 88$ & $<1$ & $<1$ & $<1$ & $<1$ & $<1$ & $<1$ & $<1$ & $<1$ & $<1$ & $0.9 \pm 0.1$ & $<1$ & $<10$ \\
\hline & $1 / 12 / 89$ & $<1$ & $<1$ & $<1$ & $<1$ & $<1$ & $<1$ & $<1$ & $<1$ & $<1$ & $0.14 \pm 0.09$ & $<1$ & $<10$ \\
\hline & $8 / 15 / 89$ & $<1$ & $<1$ & $<1$ & $<1$ & $<1$ & $<1$ & $<1$ & $<1$ & $<1$ & $0.8 \pm 0.1$ & $<1$ & $<10$ \\
\hline & $10 / 16 / 89$ & $<1$ & $<1$ & $<1$ & $<1$ & $<1$ & $<1$ & $<1$ & $<1$ & $<1$ & $0.5 \pm 0.2$ & $<1$ & $<10$ \\
\hline
\end{tabular}




\begin{tabular}{|c|c|c|c|c|c|c|c|c|c|c|c|}
\hline $\begin{array}{l}\text { Site } \\
\text { No. }\end{array}$ & $\begin{array}{c}\text { Collection } \\
\text { Date }\end{array}$ & $\begin{array}{c}\mathrm{Lu} \\
(u g / L)\end{array}$ & $\begin{array}{c}M g \\
(m g / L)\end{array}$ & $\begin{array}{c}M n \\
(u g / L)\end{array}$ & $\begin{array}{c}\text { Mo } \\
\text { (ug/L) }\end{array}$ & $\begin{array}{c}\mathrm{Na} \\
(\mathrm{mg} / \mathrm{L})\end{array}$ & $\begin{array}{c}N b \\
(u g / L)\end{array}$ & $\begin{array}{c}\text { Nd } \\
(u g / L)\end{array}$ & $\begin{array}{c}N i \\
(u g / L)\end{array}$ & $\begin{array}{l}\mathrm{NO}_{3}-\mathrm{N} \\
(\mathrm{mg} / \mathrm{L})\end{array}$ & $\begin{array}{c}\text { Os } \\
(u g / L)\end{array}$ \\
\hline \multirow[t]{2}{*}{$M V-5$} & $8 / 15 / 89$ & $<1$ & $0.8 \pm 0.1$ & $50 \pm 10$ & $<1$ & $0.9 \pm 0.1$ & $<1$ & $<1$ & $120 \pm 15$ & $<0.2$ & $<1$ \\
\hline & $10 / 16 / 89$ & $<1$ & $1.1 \pm 0.1$ & $100 \pm 10$ & $<1$ & $1.2 \pm 0.1$ & $<1$ & $<1$ & $<50$ & $0.4 \pm 0.1$ & $<1$ \\
\hline \multirow[t]{3}{*}{$M V-11$} & $9 / 15 / 88$ & $<1$ & $0.9 \pm 0.1$ & $55 \pm 11$ & $<i$ & $0.8 \pm 0.1$ & $<1$ & $<1$ & $120 \pm 24$ & $<0.2$ & $<1$ \\
\hline & $8 / 15 / 89$ & $<1$ & $0.7 \pm 0.1$ & $80 \pm 20$ & $<1$ & $0.8 \pm 0.1$ & $<1$ & $<1$ & $90 \pm 20$ & $<0.2$ & $<1$ \\
\hline & $10 / 16 / 89$ & $<1$ & $0.8 \pm 0.1$ & $50 \pm 10$ & $<1$ & $0.7 \pm 0.1$ & $<1$ & $<1$ & $100 \pm 20$ & $<0.2$ & $<1$ \\
\hline \multirow[t]{3}{*}{$M V-13$} & $9 / 15 / 88$ & $<1$ & $1.0 \pm 0.1$ & $<50$ & $<1$ & $0.8 \pm 0.1$ & $<1$ & $<1$ & $<1$ & $<0.2$ & $<1$ \\
\hline & $9 / 13 / 89$ & $<1$ & $1.2 \pm 0.1$ & $<50$ & $<1$ & $0.8 \pm 0.1$ & $<1$ & $<1$ & $<1$ & $<0.2$ & $<1$ \\
\hline & $10 / 16 / 89$ & $<1$ & $1.3 \pm 0.1$ & $<50$ & $<1$ & $0.9 \pm 0.1$ & $<1$ & $<1$ & $<1$ & $<0.2$ & $<1$ \\
\hline \multirow[t]{2}{*}{$M V-14 A$} & $8 / 15 / 89$ & $<1$ & $1.1 \pm 0.1$ & $<50$ & $<1$ & $0.9 \pm 0.1$ & $<1$ & $<1$ & $40 \pm 20$ & $<0.2$ & $<1$ \\
\hline & $10 / 16 / 89$ & $<1$ & $1.0 \pm 0.1$ & $<50$ & $<1$ & $0.8 \pm 0.1$ & $<1$ & $<1$ & $<10$ & $<0.2$ & $<1$ \\
\hline \multirow[t]{3}{*}{$M V-14 B$} & $9 / 13 / 89$ & $<1$ & $1.4 \pm 0.1$ & $<50$ & $<1$ & $1.1 \pm 0.1$ & $<1$ & $<1$ & $<1$ & $<0.2$ & $<1$ \\
\hline & $10 / 5 / 89$ & $<1$ & $1.5 \pm 0.1$ & $<50$ & $<1$ & $0.6 \pm 0.1$ & $<1$ & $<1$ & $<1$ & $<0.2$ & $<1$ \\
\hline & $10 / 16 / 89$ & $<1$ & $1.0 \pm 0.1$ & $<50$ & $<1$ & $0.9 \pm 0.1$ & $<1$ & $<1$ & $<1$ & $<0.2$ & $<1$ \\
\hline$M V-20$ & $8 / 15 / 89$ & $<1$ & $1.8 \pm 0.2$ & $<50$ & $<1$ & $0.6 \pm 0.1$ & $<1$ & $<1$ & $<1$ & $<0.2$ & $<1$ \\
\hline \multirow[t]{4}{*}{$M V-25$} & $9 / 15 / 88$ & $<1$ & $1.0 \pm 0.1$ & $70 \pm 14$ & $<1$ & $0.6 \pm 0.1$ & $<1$ & $<1$ & $<1$ & $<0.2$ & $<1$ \\
\hline & $8 / 15 / 89$ & $<1$ & $1.3 \pm 0.1$ & $90 \pm 15$ & $<1$ & $0.8 \pm 0.1$ & $<1$ & $<1$ & $<1$ & $<0.2$ & $<1$ \\
\hline & $9 / 13 / 89$ & $<1$ & $1.3 \pm 0.1$ & $70 \pm 10$ & $<1$ & $0.8 \pm 0.1$ & $<1$ & $<1$ & $<1$ & $<0.2$ & $<1$ \\
\hline & $10 / 16 / 89$ & $<1$ & $1.1 \pm 0.1$ & $60 \pm 10$ & $<1$ & $0.7 \pm 0.1$ & $<1$ & $<1$ & $<1$ & $<0.2$ & $<1$ \\
\hline \multirow[t]{3}{*}{$M V-35$} & $9 / 15 / 88$ & $<1$ & $0.6 \pm 0.1$ & $54 \pm 10$ & $<1$ & $0.4 \pm 0.1$ & $<1$ & $<1$ & $<1$ & $<0.2$ & $<1$ \\
\hline & $8 / 15 / 89$ & $<1$ & $0.9 \pm 0.1$ & $60 \pm 10$ & $<1$ & $0.5 \pm 0.1$ & $<1$ & $<1$ & $<1$ & $<0.2$ & $<1$ \\
\hline & $10 / 16 / 89$ & $<1$ & $0.7 \pm 0.1$ & $50 \pm 10$ & $<1$ & $0.4 \pm 0.1$ & $<1$ & $<1$ & $<1$ & $<0.2$ & $<1$ \\
\hline \multirow[t]{2}{*}{$S F-2$} & $10 / 5 / 89$ & $<1$ & $0.6 \pm 0.1$ & $<50$ & $<1$ & $0.4 \pm 0.1$ & $<1$ & $<1$ & $<1$ & $<0.2$ & $<1$ \\
\hline & $10 / 16 / 89$ & $<1$ & $0.8 \pm 0.1$ & $<50$ & $<1$ & $0.4 \pm 0.1$ & $<1$ & $<1$ & $<1$ & $<0.2$ & $<1$ \\
\hline \multirow[t]{7}{*}{$S F-8$} & $8 / 21 / 88$ & -10 & $0.73 \pm 0.11$ & $100 \pm 10$ & $<1$ & $0.8 \pm 0.1$ & $<1$ & $<1$ & $100 \pm 10$ & $<0.2$ & $<1$ \\
\hline & $9 / 15 / 88$ & $<1$ & $0.5 \pm 0.1$ & $<50$ & $<1$ & $0.3 \pm 0.1$ & $<1$ & $<1$ & $<1$ & $<0.2$ & $<1$ \\
\hline & $9 / 25 / 88$ & $<1$ & $0.8 \pm 0.1$ & $<50$ & $<1$ & $0.5 \pm 0.1$ & $<1$ & $<1$ & $<50$ & $<0.2$ & $<1$ \\
\hline & $1 / 12 / 89$ & $<10$ & $0.10 \pm 0.04$ & $6 \pm 1$ & $<1$ & $0.12 \pm 0.04$ & $<1$ & $<1$ & $<1$ & $0.6 \pm 0.1$ & $<1$ \\
\hline & $8 / 15 / 89$ & $<1$ & $0.7 \pm 0.1$ & $<50$ & $<1$ & $0.5 \pm 0.1$ & $<1$ & $<1$ & $<1$ & $<0.2$ & $<1$ \\
\hline & $10 / 05 / 89$ & $<1$ & $0.6 \pm 0.1$ & $<50$ & $<1$ & $0.6 \pm 0.1$ & $<1$ & $<1$ & $<1$ & $<0.2$ & $<1$ \\
\hline & $10 / 16 / 89$ & $<1$ & $0.5 \pm 0.1$ & $<50$ & $<1$ & $0.3 \pm 0.2$ & $<1$ & $<1$ & $<1$ & $<0.2$ & $<1$ \\
\hline
\end{tabular}




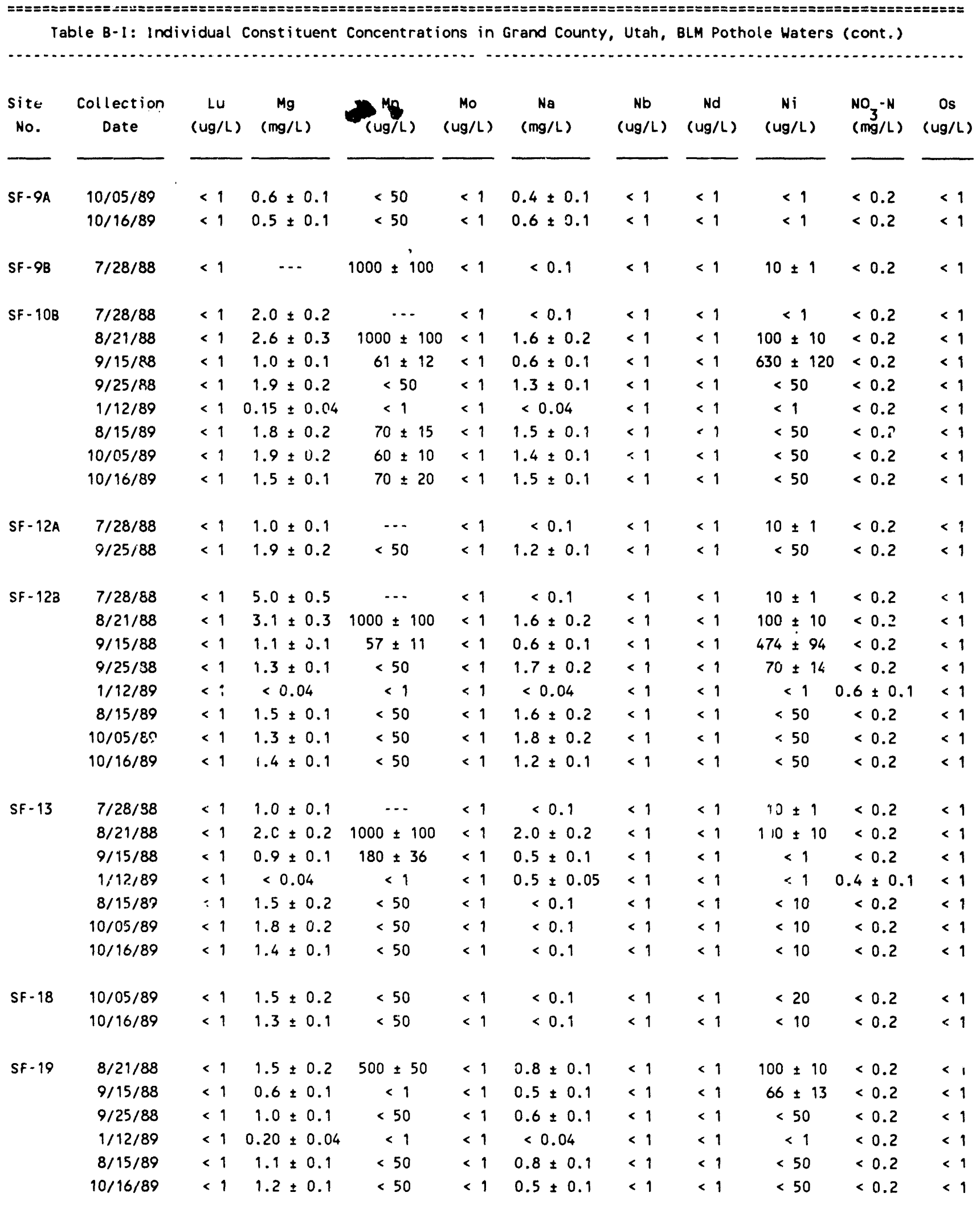




\begin{tabular}{|c|c|c|c|c|c|c|c|c|c|c|c|c|}
\hline $\begin{array}{l}\text { Site } \\
\text { No. }\end{array}$ & $\begin{array}{c}\text { Collection } \\
\text { Date }\end{array}$ & $\begin{array}{c}P K \\
(u g / L)\end{array}$ & $\begin{array}{c}P d \\
(u g / L)\end{array}$ & $\begin{array}{l}\mathrm{PO}_{4}-\mathrm{P} \\
(\mathrm{mg} / \mathrm{L})\end{array}$ & $\begin{array}{c}\mathrm{Pr} \\
(u g / L)\end{array}$ & $\begin{array}{c}P t \\
(u g / L)\end{array}$ & $\begin{array}{c}\mathrm{Rb} \\
(\mathrm{ug} / \mathrm{L})\end{array}$ & $\begin{array}{c}\operatorname{Re} \\
(u g / L)\end{array}$ & $\begin{array}{c}\mathrm{Rh} \\
(\mathrm{ug} / \mathrm{L})\end{array}$ & $\begin{array}{c}R u \\
(u g / L)\end{array}$ & $\begin{array}{c}\mathrm{sb} \\
(u g / L)\end{array}$ & $\begin{array}{c}\text { Sc } \\
(u g / L)\end{array}$ \\
\hline \multirow[t]{2}{*}{$M V-5$} & $8 / 15 / 89$ & $=1$ & $<1$ & $<0.2$ & $<1$ & $<1$ & $<1$ & $<1$ & $<1$ & $<1$ & $<1$ & $<1$ \\
\hline & $10 / 16 / 89$ & $<1$ & $<1$ & $<0.2$ & $<1$ & $<1$ & $<1$ & $<1$ & $<1$ & $<1$ & $<1$ & $<1$ \\
\hline \multirow[t]{3}{*}{$M V-11$} & $9 / 15 / 88$ & $<1$ & $<1$ & $<0.2$ & $<1$ & $<1$ & $<1$ & $<1$ & $<1$ & $<1$ & $<1$ & $<1$ \\
\hline & $8 / 15 / 89$ & $<1$ & $<1$ & $<0.2$ & $<1$ & $<1$ & $<1$ & $<1$ & $<1$ & $<1$ & $<1$ & $<1$ \\
\hline & $10 / 16 / 89$ & $<1$ & $<1$ & $<0.2$ & $<1$ & $<1$ & $<1$ & $<1$ & $<1$ & $<1$ & $<1$ & $<1$ \\
\hline \multirow[t]{3}{*}{$M V-13$} & $9 / 15 / 88$ & $<1$ & $<1$ & $<0.2$ & $<1$ & $<1$ & $<1$ & $<1$ & $<1$ & $<1$ & $<1$ & $<1$ \\
\hline & $9 / 13 / 89$ & $<1$ & $<1$ & $<0.2$ & $<1$ & $<1$ & $<1$ & $<1$ & $<1$ & $<1$ & $<1$ & $<1$ \\
\hline & $10 / 16 / 89$ & $<1$ & $<1$ & $<0.2$ & $<1$ & $<1$ & $<1$ & $<1$ & $<1$ & $<1$ & $<1$ & $<1$ \\
\hline \multirow[t]{2}{*}{$M V-14 A$} & $8 / 15 / 89$ & $<1$ & $<1$ & $<0.2$ & $<1$ & $<1$ & $<1$ & $<1$ & $<1$ & $<1$ & $<1$ & $<1$ \\
\hline & $10 / 16 / 89$ & $<1$ & $<1$ & $<0.2$ & $<1$ & $<1$ & $<1$ & $<1$ & $<1$ & $<1$ & $<1$ & $<1$ \\
\hline \multirow[t]{3}{*}{$M V-14 B$} & $9 / 13 / 89$ & $<1$ & $<1$ & $<0.2$ & $<1$ & $<1$ & $<1$ & $<1$ & $<1$ & $<1$ & $<1$ & $<1$ \\
\hline & $10 / 5 / 89$ & $<1$ & $<1$ & $<0.2$ & $<1$ & $<1$ & $<1$ & $<1$ & $<1$ & $<1$ & $<1$ & $<1$ \\
\hline & $10 / 16 / 89$ & $<1$ & $<1$ & $<0.2$ & $<1$ & $<1$ & $<1$ & $<1$ & $<1$ & $<1$ & $<1$ & $<1$ \\
\hline$M V-20$ & $8 / 15 / 89$ & $<1$ & $<1$ & $<0.2$ & $<1$ & $<1$ & $<1$ & $<1$ & $<1$ & $<1$ & $<1$ & $<1$ \\
\hline \multirow[t]{4}{*}{$M V-25$} & $9 / 15 / 88$ & $<1$ & $<1$ & $<0.2$ & $<1$ & $<1$ & $<1$ & $<1$ & $<1$ & $<1$ & $<1$ & $<1$ \\
\hline & $8 / 15 / 89$ & $<1$ & $<1$ & $<0.2$ & $<1$ & $<1$ & $<1$ & $<1$ & $<1$ & $<1$ & $<1$ & $<1$ \\
\hline & $9 / 13 / 89$ & $<1$ & $<1$ & $<0.2$ & $<1$ & $<1$ & $<1$ & $<1$ & $<1$ & $<1$ & $<1$ & $<1$ \\
\hline & $10 / 16 / 89$ & $<1$ & $<1$ & $<0.2$ & $<1$ & $<1$ & $<1$ & $<1$ & $<1$ & $<1$ & $<1$ & $<1$ \\
\hline \multirow[t]{3}{*}{$M V-35$} & $9 / 15 / 88$ & $<1$ & $<1$ & $<0.2$ & $<:$ & $<1$ & $<1$ & $<1$ & $<1$ & $<1$ & $<1$ & $<1$ \\
\hline & $8 / 15 / 89$ & $<1$ & $<1$ & $<0.2$ & $<1$ & $<1$ & $<1$ & $<1$ & $<1$ & $<:$ & $<1$ & $<1$ \\
\hline & $10 / 16 / 89$ & $<1$ & $<1$ & $<0.2$ & $<1$ & $<1$ & $<1$ & $<1$ & $<1$ & $<1$ & $<1$ & $<1$ \\
\hline \multirow[t]{2}{*}{$S F-2$} & $10 / 5 / 89$ & $<1$ & $<1$ & $<0.2$ & $<1$ & $<1$ & $<1$ & $<1$ & $<1$ & $<1$ & $<1$ & $<1$ \\
\hline & $10 / 16 / 89$ & $<1$ & $<1$ & $<0.2$ & $<1$ & $<1$ & $<1$ & $<1$ & $<1$ & $<1$ & $<1$ & $<1$ \\
\hline \multirow[t]{7}{*}{$S F-8$} & $8 / 21 / 88$ & $<1$ & $<1$ & $<0.2$ & $<1$ & $<1$ & $<1$ & $<1$ & $<1$ & $<1$ & $<1$ & $<1$ \\
\hline & $9 / 15 / 88$ & $<1$ & $<1$ & $<0.2$ & $<1$ & $<1$ & $<1$ & $<1$ & $<1$ & $<1$ & $<1$ & $<1$ \\
\hline & $9 / 25 / 88$ & $<1$ & $<1$ & $<0.2$ & $<1$ & $<1$ & $<1$ & $<1$ & $<1$ & $<1$ & $<1$ & $<1$ \\
\hline & $1 / 12 / 89$ & $<1$ & $<1$ & $<0.2$ & $<1$ & $<1$ & $<1$ & $<1$ & $<1$ & $<1$ & $<1$ & $<1$ \\
\hline & $8 / 15 / 89$ & $<1$ & $<1$ & $<0.2$ & $<1$ & $<1$ & $<1$ & $<1$ & $<1$ & $<1$ & $<1$ & $<1$ \\
\hline & $10 / 05 / 89$ & $<1$ & $<1$ & $<0.2$ & $<1$ & $<1$ & $<1$ & $<1$ & $<1$ & $<1$ & $<1$ & $<1$ \\
\hline & $10 / 16 / 89$ & $<1$ & $<1$ & $<0.2$ & $<1$ & $<1$ & $: 1$ & $<1$ & $<1$ & $<1$ & $<1$ & $<1$ \\
\hline
\end{tabular}




\begin{tabular}{|c|c|c|c|c|c|c|c|c|c|c|c|c|c|c|c|c|c|c|c|}
\hline \multirow{3}{*}{$\begin{array}{l}\text { Site } \\
\text { No. } \\
\text { SF-OA }\end{array}$} & \multirow{2}{*}{$\begin{array}{c}\begin{array}{c}\text { Collection } \\
\text { Date }\end{array} \\
10 / 05 / 89\end{array}$} & \multirow{2}{*}{$\begin{array}{c}\mathrm{Pb} \\
(\mathrm{ug} / \mathrm{L}) \\
<1\end{array}$} & $\begin{array}{c}P d \\
(u g / L)\end{array}$ & $\begin{array}{l}\mathrm{PO}_{4}-\mathrm{P} \\
(\mathrm{mg} / \mathrm{L})\end{array}$ & \multicolumn{2}{|c|}{$\begin{array}{c}P r \\
(u g / L)\end{array}$} & \multicolumn{2}{|c|}{$\begin{array}{c}P t \\
(u g / L)\end{array}$} & \multicolumn{2}{|c|}{$\begin{array}{c}R b \\
(u g / L)\end{array}$} & \multicolumn{2}{|c|}{$\begin{array}{c}\operatorname{Re} \\
(u g / L)\end{array}$} & \multicolumn{2}{|c|}{$\begin{array}{c}\mathrm{Rh} \\
\text { (ug/L) }\end{array}$} & \multicolumn{2}{|c|}{$\begin{array}{c}R u \\
(u g / L)\end{array}$} & \multicolumn{2}{|c|}{$\begin{array}{c}\text { Sb } \\
(u g / L)\end{array}$} & \multirow{2}{*}{$\begin{array}{c}\text { Sc } \\
(u g / L) \\
<1\end{array}$} \\
\hline & & & $<1$ & $<0.2$ & $<$ & 1 & & 1 & & 1 & & 1 & & 1 & & 1 & & 1 & \\
\hline & $10 / 16 / 89$ & $<1$ & $<1$ & $<0.2$ & $<$ & 1 & & 1 & & 1 & & 1 & & 1 & & 1 & & 1 & $<1$ \\
\hline$S F-9 B$ & $7 / 28 / 88$ & $<1$ & $<1$ & $<0.2$ & $<$ & 1 & & 1 & & 1 & $<$ & 1 & $<$ & 1 & & 1 & $<$ & 1 & $1000 \pm 100$ \\
\hline \multirow[t]{8}{*}{$S F-10 B$} & $7 / 28 / 88$ & $<1$ & $<1$ & $<0.2$ & $<$ & 1 & & 1 & & 1 & $<$ & 1 & $<$ & 1 & & 1 & $<$ & 1 & $1000 \pm 100$ \\
\hline & $8 / 21 / 88$ & $10 \pm 1$ & $<1$ & $<0.2$ & $<$ & 1 & & 1 & & 1 & $<$ & 1 & $<$ & 1 & & 1 & $<$ & 1 & $<1$ \\
\hline & $9 / 15 / 88$ & $<1$ & $<1$ & $<0.2$ & $<$ & 1 & & 1 & $<$ & 1 & $<$ & 1 & $<$ & 1 & & 1 & $<$ & 1 & $106 \pm 21$ \\
\hline & $9 / 25 / 88$ & $<1$ & $<1$ & $<0.2$ & $<$ & 1 & & 1 & $<$ & 1 & $<$ & 1 & $<$ & 1 & & 1 & $<$ & 1 & $<1$ \\
\hline & $1 / 12 / 89$ & $<1$ & $<1$ & $<0.2$ & $<$ & 1 & & 1 & $<$ & 1 & $<$ & 1 & $<$ & 1 & & 1 & $<$ & 1 & $<1$ \\
\hline & $8 / 15 / 89$ & $<1$ & $<1$ & $<0.2$ & $<$ & 1 & & 1 & $<$ & 1 & $<$ & 1 & $<$ & 1 & & 1 & $<$ & 1 & $<1$ \\
\hline & $10 / 05 / 89$ & $<1$ & $<1$ & $<0.2$ & $<$ & 1 & & 1 & $<$ & 1 & $<$ & 1 & $<$ & 1 & $<$ & 1 & $<$ & 1 & $<1$ \\
\hline & $10 / 16 / 89$ & $<1$ & $<1$ & $<0.2$ & $<$ & 1 & & 1 & $<$ & 1 & $<$ & 1 & $<$ & 1 & $<$ & 1 & $<$ & 1 & $<1$ \\
\hline \multirow[t]{2}{*}{$S F-12 A$} & $7 / 28 / 88$ & $<1$ & $<1$ & $<0.2$ & $<$ & 1 & & 1 & $<$ & 1 & $<$ & 1 & $<$ & 1 & $<$ & & $<$ & 1 & $1000 \pm 100$ \\
\hline & $9 / 25 / 88$ & $<1$ & $<1$ & $<0.2$ & $<$ & 1 & & 1 & $<$ & 1 & $<$ & 1 & $<$ & 1 & $<$ & 1 & $<$ & 1 & $<1$ \\
\hline \multirow[t]{8}{*}{$S F-12 B$} & $7 / 28 / 88$ & $<1$ & $<1$ & $<0.2$ & $<$ & 1 & & 1 & $<$ & 1 & $<$ & 1 & $<$ & 1 & $<$ & & $<$ & 1 & $1000 \pm 100$ \\
\hline & $8 / 21 / 88$ & $5.0 \pm 0.5$ & $<1$ & $<0.2$ & $<$ & 1 & $<$ & 1 & $<$ & 1 & $<$ & 1 & $<$ & 1 & $<$ & 1 & $<$ & 1 & $<1$ \\
\hline & $9 / 15 / 88$ & $<1$ & $<1$ & $<0.2$ & $<$ & 1 & $<$ & 1 & $<$ & 1 & $<$ & 1 & $<$ & 1 & $<$ & 1 & $<$ & 1 & $<1$ \\
\hline & $9 / 25 / 88$ & $<1$ & $<1$ & $<0.2$ & $<$ & 1 & $<$ & 1 & $<$ & 1 & $<$ & 1 & $<$ & 1 & $<$ & 1 & $<$ & 1 & $<1$ \\
\hline & $1 / 12 / 89$ & $<1$ & $<1$ & $<0.2$ & $<$ & 1 & $<$ & 1 & $<$ & 1 & $<$ & 1 & $<$ & 1 & $<$ & 1 & $<$ & 1 & $<1$ \\
\hline & $8 / 15 / 89$ & $<1$ & $<1$ & $<0.2$ & $<$ & 1 & $<$ & 1 & $<$ & 1 & $<$ & 1 & $<$ & 1 & $<$ & 1 & $<$ & 1 & $<1$ \\
\hline & $10 / 05 / 89$ & $<1$ & $<1$ & $<0.2$ & $<$ & 1 & $<$ & $\hat{i}$ & $<$ & 1 & $<$ & 1 & $<$ & 1 & $<$ & 1 & $<$ & 1 & $<1$ \\
\hline & $10 / 16 / 8$ & $<1$ & $<1$ & $<0.2$ & $<$ & 1 & $<$ & 1 & $<$ & 1 & $<$ & 1 & $<$ & 1 & $<$ & 1 & $<$ & 1 & $<1$ \\
\hline \multirow[t]{7}{*}{$S F-13$} & $7 / 28 / 88$ & $<1$ & $<1$ & $<0.2$ & $<$ & 1 & $<$ & 1 & $<$ & 1 & $<$ & 1 & $<$ & 1 & $<$ & 1 & $<$ & 11 & $1000 \pm 100$ \\
\hline & $8 / 21 / 88$ & $5 \pm 1$ & $<1$ & $<0.2$ & $<$ & 1 & $<$ & 1 & $<$ & 1 & $<$ & 1 & $<$ & 1 & $<$ & 1 & $<$ & 1 & $<1$ \\
\hline & $9 / 15 / 88$ & $<1$ & $<1$ & $<0.2$ & $<$ & 1 & $<$ & 1 & $<$ & 1 & $<$ & 1 & $<$ & 1 & $<$ & 1 & $<$ & 1 & $<1$ \\
\hline & $1 / 12 / 89$ & $<1$ & $<1$ & $<0.2$ & $<$ & 1 & $<$ & 1 & $<$ & 1 & $<$ & 1 & $<$ & 1 & $<$ & 1 & $<$ & 1 & $<1$ \\
\hline & $8 / 15 / 89$ & $<1$ & $<1$ & $<0.2$ & $<$ & 1 & $<$ & 1 & $<$ & 1 & $<$ & 1 & $<$ & 1 & $<$ & 1 & 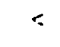 & 1 & $<1$ \\
\hline & $10 / 05 / 89$ & $<1$ & $<1$ & $<0.2$ & $<$ & 1 & $<$ & 1 & $<$ & 1 & $<$ & 1 & $<$ & 1 & $<$ & 1 & $<$ & 1 & $<\uparrow$ \\
\hline & $10 / 16 / 89$ & $<1$ & $<1$ & $<0.2$ & $<$ & 1 & $<$ & 1 & $<$ & 1 & $<$ & 1 & $<$ & 1 & $<$ & 1 & $<$ & 1 & $<1$ \\
\hline \multirow[t]{2}{*}{$S F-18$} & $10 / 05 / 89$ & $<1$ & $<1$ & $<0.2$ & $<$ & 1 & $<$ & 1 & $<$ & 1 & $<$ & 1 & $<$ & 1 & $<$ & 1 & $<$ & 1 & $<1$ \\
\hline & $10 / 16 / 89$ & $<1$ & $<1$ & $<0.2$ & $<$ & 1 & $<$ & 1 & $<$ & 1 & $<$ & 1 & $<$ & 1 & $<$ & 1 & $<$ & 1 & $<1$ \\
\hline \multirow[t]{6}{*}{$S F-19$} & $8 / 21 / 88$ & $5 \pm 1$ & $<1$ & $<0.2$ & $<$ & 1 & $<$ & 1 & $<$ & 1 & $<$ & 1 & $<$ & 1 & $<$ & 1 & $<$ & 1 & $<1$ \\
\hline & $9 / 15 / 88$ & $<1$ & $<1$ & $<0.2$ & $<$ & 1 & $<$ & 1 & $<$ & 1 & $<$ & 1 & $<$ & 1 & $<$ & 1 & $<$ & 1 & $<1$ \\
\hline & $9 / 25 / 88$ & $<1$ & $<1$ & $<0.2$ & $<$ & 1 & $<$ & 1 & $<$ & 1 & $<$ & 1 & $<$ & 1 & $<$ & 1 & $<$ & 1 & $<1$ \\
\hline & $1 / 12 / 89$ & $<1$ & $<1$ & $<0.2$ & $<$ & 1 & $<$ & 1 & $<$ & 1 & $<$ & 1 & $<$ & 1 & $<$ & 1 & $<$ & 1 & $<1$ \\
\hline & $8 / 15 / 89$ & $<1$ & $<1$ & $<0.2$ & $<$ & 1 & $<$ & 1 & $<$ & 1 & $<$ & 1 & $<$ & 1 & $<$ & 1 & $<$ & 1 & $<1$ \\
\hline & $10 / 16 / 89$ & $<1$ & $<1$ & $<0.2$ & $<$ & 1 & $<$ & 1 & $<$ & 1 & $<$ & 1 & $<$ & 1 & $<$ & 1 & $<$ & 1 & $<1$ \\
\hline
\end{tabular}




\begin{tabular}{|c|c|c|c|c|c|c|c|c|c|c|c|}
\hline $\begin{array}{l}\text { Site } \\
\text { No. }\end{array}$ & $\begin{array}{c}\text { Collection } \\
\text { Date }\end{array}$ & $\begin{array}{c}\text { Se } \\
(u g / L)\end{array}$ & $\begin{array}{c}\mathrm{Sm} \\
(\mathrm{ug} / \mathrm{L})\end{array}$ & $\begin{array}{c}S n \\
(u g / L)\end{array}$ & $\left.{ }_{(\mathrm{mg} / \mathrm{L}}^{\mathrm{SO}}\right)$ & $\begin{array}{c}S r \\
(u g / L)\end{array}$ & $\begin{array}{c}\mathrm{Ta} \\
(\mathrm{ug} / \mathrm{L})\end{array}$ & $\begin{array}{l}\text { T Alk.* } \\
(\mathrm{mg} / \mathrm{L})\end{array}$ & $\begin{array}{c}\text { Tb } \\
(u g / L)\end{array}$ & $\begin{array}{c}\text { Te } \\
(u g / L)\end{array}$ & $\begin{array}{c}\text { Th } \\
\text { (ug/L) }\end{array}$ \\
\hline \multirow[t]{2}{*}{$M V-5$} & $8 / 15 / 89$ & $<10$ & $<1$ & $<1$ & $8.1 \pm 0.8$ & $80 \pm 20$ & $<1$ & --- & $<1$ & $<1$ & $<1$ \\
\hline & $10 / 16 / 89$ & $<10$ & $<1$ & $<1$ & $6.9 \pm 0.7$ & $400 \pm 40$ & $<1$ & $\cdots$ & $<1$ & $<1$ & $<1$ \\
\hline \multirow[t]{3}{*}{$M V-11$} & $9 / 15 / 88$ & $<10$ & $<1$ & $<1$ & $8.7 \pm 0.9$ & $80 \pm 16$ & $<1$ & $25.2 \pm 1.5$ & $<1$ & $<1$ & $<1$ \\
\hline & $8 / 15 / 89$ & $<10$ & $<1$ & $<1$ & $8.1 \pm 0.8$ & $90 \pm 20$ & $<1$ & --- & $<1$ & $<1$ & $<1$ \\
\hline & $10 / 16 / 89$ & $<10$ & $<1$ & $<1$ & $8.1 \pm 0.8$ & $70 \pm 25$ & $<1$ & $-\cdots$ & $<1$ & $<1$ & $<1$ \\
\hline \multirow[t]{3}{*}{$M V-13$} & $9 / 15 / 88$ & $<10$ & $<1$ & $<1$ & $8.2 \pm 0.8$ & $130 \pm 26$ & $<1$ & $22.6 \pm 1.4$ & $<1$ & $<1$ & $<1$ \\
\hline & $9 / 13 / 89$ & $<10$ & $<1$ & $<1$ & $7.1 \pm 0.7$ & $80 \pm 30$ & $<1$ & --- & $<1$ & $<1$ & $<1$ \\
\hline & $10 / 16 / 89$ & $<10$ & $<1$ & $<1$ & $8.5 \pm 0.8$ & $100 \pm 20$ & $<1$ & $\cdots$ & $<1$ & $<1$ & $<1$ \\
\hline \multirow[t]{2}{*}{$M V-14 A$} & $8 / 15 / 89$ & $<10$ & $<1$ & $<1$ & $8.6 \pm 0.8$ & $100 \pm 20$ & $<1$ & $\cdots$ & $<1$ & $<1$ & $<1$ \\
\hline & $10 / 16 / 89$ & $<10$ & $<1$ & $<1$ & $8.9 \pm 0.9$ & $80 \pm 20$ & $<1$ & -- & $<1$ & $<1$ & $<1$ \\
\hline \multirow[t]{3}{*}{$M V-14 B$} & $9 / 13 / 89$ & $<10$ & $<1$ & $<1$ & $9.1 \pm 0.9$ & $180 \pm 30$ & $<1$ & $\cdots$ & $<1$ & $<1$ & $<1$ \\
\hline & $10 / 5 / 89$ & $<10$ & $<1$ & $<1$ & $70 \pm 0.7$ & $150 \pm 20$ & $<1$ & -- & $<1$ & $<1$ & $<1$ \\
\hline & $10 / 16 / 89$ & $<10$ & $<1$ & $<1$ & $8.1 \pm 0.8$ & $70 \pm 20$ & $<1$ & -- & $<1$ & $<1$ & $<1$ \\
\hline$M V-20$ & $8 / 15 / 89$ & $<10$ & $<1$ & $<1$ & $7.6 \pm 0.8$ & $100 \pm 20$ & $<1$ & --- & $<1$ & $<1$ & $<1$ \\
\hline \multirow[t]{4}{*}{$M V-25$} & $9 / 15 / 88$ & $<10$ & $<1$ & $<1$ & $6.3 \pm 0.6$ & $85 \pm 17$ & $<1$ & $31.8 \pm 1.9$ & $<1$ & $<1$ & $<1$ \\
\hline & $8 / 15 / 89$ & $<10$ & $<1$ & $<1$ & $7.1 \pm 0.7$ & $90 \pm 20$ & $<1$ & -- & $<1$ & $<1$ & $<1$ \\
\hline & $9 / 13 / 89$ & $<10$ & $<1$ & $<1$ & $6.6 \pm 0.7$ & $70 \pm 20$ & $<1$ & -- & $<1$ & $<1$ & $<1$ \\
\hline & $10 / 16 / 89$ & $<10$ & $<1$ & $<1$ & $7.5 \pm 0.8$ & $70 \pm 20$ & $<1$ & -- & $<1$ & $<1$ & $<1$ \\
\hline \multirow[t]{3}{*}{$M V-35$} & $9 / 15 / 88$ & $<10$ & $<1$ & $<1$ & $6.9 \pm 0.7$ & $80 \pm 16$ & $<1$ & $13.4 \pm 0.8$ & $<1$ & $<1$ & $<1$ \\
\hline & $8 / 15 / 89$ & $<10$ & $<1$ & $<1$ & $7.2 \pm 0.7$ & $80 \pm 20$ & $<1$ & -- & $<1$ & $<1$ & $<1$ \\
\hline & $10 / 16 / 89$ & $<10$ & $<1$ & $<1$ & $6.1 \pm 0.6$ & $70 \pm 20$ & $<1$ & -- & $<1$ & $<1$ & $<1$ \\
\hline \multirow[t]{2}{*}{$S F-2$} & $10 / 5 / 89$ & $<10$ & $<1$ & $<1$ & $4.4 \pm 0.4$ & $<1$ & $<1$ & $\cdots$ & $<1$ & $<1$ & $<1$ \\
\hline & $10 / 16 / 89$ & $<10$ & $<1$ & $<1$ & $4.3 \pm 0.4$ & $<1$ & $<1$ & $\cdots$ & $<1$ & $<1$ & $<1$ \\
\hline \multirow[t]{7}{*}{$S F-8$} & $8 / 21 / 88$ & $50 \pm 5$ & $<1$ & $<1$ & $7.9 \pm 0.8$ & $100 \pm 10$ & $<1$ & $33.1 \pm 3.3$ & $<1$ & $<1$ & $<1$ \\
\hline & $9 / 15 / 88$ & $<10$ & $<1$ & $<1$ & $4.0 \pm 0.4$ & $<1$ & $<1$ & $9.2 \pm 0.6$ & $<1$ & $<1$ & $<2$ \\
\hline & $9 / 25 / 88$ & $<10$ & $<1$ & $<1$ & $8.0 \pm 0.8$ & $88 \pm 17$ & $<1$ & $17.6 \pm 1.1$ & $<1$ & $<1$ & $<1$ \\
\hline & $1 / 12 / 89$ & $<10$ & $<1$ & $<1$ & $0.4 \pm 0.1$ & $<1$ & $<1$ & $2.1 \pm 0.2$ & $<1$ & $<1$ & $<1$ \\
\hline & $8 / 15 / 89$ & $<10$ & $<1$ & $<1$ & $7.4 \pm 0.7$ & $100 \pm 10$ & $<1$ & -- & $<1$ & $<1$ & $<1$ \\
\hline & $10 / 05 / 89$ & $<10$ & $<1$ & $<1$ & $6.5 \pm 0.6$ & $100 \pm 10$ & $<1$ & $-\cdots$ & $<1$ & $<1$ & $<1$ \\
\hline & $10 / 16 / 89$ & $<10$ & $<1$ & $<1$ & $6.2 \pm 0.6$ & $150 \pm 10$ & $<1$ & -- & $<1$ & $<1$ & $<1$ \\
\hline
\end{tabular}

- TAlk. = Total Alkalinity 
Table B-I: Individual Constituent Concentrations in Grand County, Utah, BLM Pothole Waters (cont.)

\begin{tabular}{|c|c|c|c|c|c|c|c|c|c|c|c|}
\hline $\begin{array}{l}\text { Site } \\
\text { No. }\end{array}$ & $\begin{array}{c}\text { Collection } \\
\text { Date }\end{array}$ & $\begin{array}{c}\mathrm{Se} \\
(\mathrm{ug} / \mathrm{L})\end{array}$ & $\begin{array}{c}\mathrm{Sm} \\
(\mathrm{ug} / \mathrm{L})\end{array}$ & $\begin{array}{c}S n \\
(u g / L)\end{array}$ & ${ }_{(\mathrm{mg} / \mathrm{L})}^{\mathrm{SO}^{4}}$ & $\begin{array}{c}S r \\
(\mathrm{lig} / L)\end{array}$ & $\begin{array}{c}T a \\
(u g / L)\end{array}$ & $\begin{array}{l}\text { T Alk.* } \\
(\mathrm{mg} / \mathrm{L})\end{array}$ & $\begin{array}{c}\text { Tb } \\
(u g / L)\end{array}$ & $\begin{array}{c}\mathrm{Te} \\
(u g / L)\end{array}$ & $\begin{array}{c}\text { Th } \\
(u g / L)\end{array}$ \\
\hline \multirow[t]{2}{*}{$S F-9 A$} & $10 / 05 / 89$ & $<10$ & $<1$ & $<1$ & $9.4 \pm 0.9$ & $200 \pm 20$ & $<1$ & $\cdots$ & $<1$ & $<1$ & $<1$ \\
\hline & $10 / 16 / 89$ & $<10$ & $<1$ & $<1$ & $9.1 \pm 0.9$ & $200 \pm 20$ & $<1$ & -- & $<1$ & $<1$ & $<1$ \\
\hline$S F-9 B$ & $7 / 28 / 88$ & $10 \pm 1$ & $<1$ & $<1$ & $15.0 \pm 1.5$ & $100 \pm 10$ & $<1$ & $57.1 \pm 5.7$ & $<1$ & $<1$ & $<1$ \\
\hline \multirow[t]{8}{*}{$S F-10 B$} & $7 / 28 / 88$ & $<10$ & $<1$ & $<1$ & $23.0 \pm 2.3$ & $500 \pm 50$ & $<1$ & $82.1 \pm 8.2$ & $<1$ & $<1$ & $<1$ \\
\hline & $8 / 21 / 88$ & $50 \pm 5$ & $<1$ & $<1$ & $18.8 \pm 1.9$ & $100 \pm 10$ & $<1$ & $68.7 \pm 6.9$ & $<1$ & $<1$ & $<1$ \\
\hline & $9 / 15 / 88$ & $<10$ & $<1$ & $<1$ & $6.3 \pm 0.6$ & $120 \pm 24$ & $<1$ & $21.1 \pm 1.3$ & $<1$ & $<1$ & $<1$ \\
\hline & $9 / 25 / 88$ & $<10$ & $<1$ & $<1$ & $14.6 \pm 1.5$ & $256 \pm 51$ & $<1$ & $49.1 \pm 2.9$ & $<1$ & $<1$ & $<1$ \\
\hline & $1 / 12 / 89$ & $<10$ & $<1$ & $<1$ & $<0.4$ & $<1$ & $<1$ & $2.3 \pm 0.2$ & $<1$ & $<1$ & $<1$ \\
\hline & $8 / 15 / 89$ & $<10$ & $<1$ & $<1$ & $9.2 \pm 0.9$ & $150 \pm 25$ & $<1$ & -- & $<1$ & $<1$ & $<1$ \\
\hline & $10 / 05 / 89$ & $<10$ & $<1$ & $<1$ & $8.5 \pm 0.9$ & $180 \pm 30$ & $<1$ & $\ldots$ & $<1$ & $<1$ & $<1$ \\
\hline & $10 / 16 / 89$ & $<10$ & $<1$ & $<1$ & $7.3 \pm 0.7$ & $120 \pm 20$ & $<1$ & $-\cdots$ & $<1$ & $<1$ & $<1$ \\
\hline \multirow[t]{2}{*}{$S F-12 A$} & $7 / 28 / 88$ & $<10$ & $<1$ & $<1$ & $52 \pm 5.2$ & $500 \pm 50$ & $<1$ & $79.7 \pm 8$ & $<1$ & $<1$ & $<1$ \\
\hline & $9 / 25 / 88$ & $<10$ & $<1$ & $<1$ & $15.3 \pm 1.5$ & $286 \pm 56$ & $<1$ & $47.5 \pm 2.8$ & $<1$ & $<1$ & $<1$ \\
\hline \multirow[t]{8}{*}{$S F-12 B$} & $7 / 28 / 88$ & $<10$ & $<1$ & $<1$ & $16.0 \pm 1.6$ & $500 \pm 50$ & $<1$ & $73.8 \pm 7.4$ & $<1$ & $<1$ & $<1$ \\
\hline & $8 / 21 / 88$ & $50 \pm 5$ & $<1$ & $<1$ & $18.2 \pm 1.8$ & $500 \pm 50$ & $<1$ & $77.4 \pm 7.7$ & $<1$ & $<1$ & $<1$ \\
\hline & $9 / 15 / 88$ & $<10$ & $<1$ & $<1$ & $6.6 \pm 0.7$ & $76 \pm 15$ & $<1$ & $25.6 \pm 1.5$ & $<1$ & $<1$ & $<1$ \\
\hline & $9 / 25 / 88$ & $<10$ & $<1$ & $<1$ & $20.2 \pm 2.0$ & $240 \pm 48$ & $<1$ & $42.4 \pm 2.5$ & $<1$ & $<1$ & $<1$ \\
\hline & $1 / 12 / 89$ & $<10$ & $<1$ & $<1$ & $<0.4$ & $<1$ & $<1$ & $0.6 \pm 0.1$ & $<1$ & $<1$ & $<1$ \\
\hline & $8 / 15 / 89$ & $<10$ & $<1$ & $<1$ & $20.0 \pm 2.0$ & $300 \pm 40$ & $<1$ & $-\cdots$ & $<1$ & $<1$ & $<1$ \\
\hline & $10 / 05 / 89$ & $<10$ & $<1$ & $<1$ & $15.6 \pm 1.6$ & $350 \pm 45$ & $<1$ & -- & $<1$ & $<1$ & $<1$ \\
\hline & $10 / 16 / 89$ & $<10$ & $<1$ & $<1$ & $18.2 \pm 1.8$ & $280 \pm 30$ & $<1$ & $\cdots$ & $<1$ & $<1$ & $<1$ \\
\hline \multirow[t]{7}{*}{$S F-13$} & $7 / 28 / 88$ & $<10$ & $<1$ & $<1$ & $15.6 \pm 1.6$ & $100 \pm 10$ & $<1$ & $72.6 \pm 7.3$ & $<1$ & $<1$ & $<1$ \\
\hline & $8 / 21 / 88$ & $50 \pm 5$ & $<1$ & $<1$ & $14.4 \pm 1.4$ & $500 \pm 50$ & $<1$ & $65.3 \pm 6.5$ & $<1$ & $<1$ & $<1$ \\
\hline & $9 / 15 / 88$ & $<10$ & $<1$ & $<1$ & $5.4 \pm 0.5$ & $119 \pm 24$ & $<1$ & $25.0 \pm 1.5$ & $<1$ & $<1$ & $<1$ \\
\hline & $1 / 12 / 89$ & $<10$ & $<1$ & $<1$ & $0.5 \pm 0.1$ & $<1$ & $<1$ & $5.0 \pm 0.5$ & $<1$ & $<1$ & $<1$ \\
\hline & $8 / 15 / 89$ & $<10$ & $<1$ & $<1$ & $14.9 \pm 1.5$ & $200 \pm 20$ & $<1$ & -- & $<1$ & $<1$ & $<1$ \\
\hline & $10 / 05 / 89$ & $<10$ & $<1$ & $<1$ & $13.3 \pm 1.3$ & $220 \pm 20$ & $<1$ & $\cdots$ & $<1$ & $<1$ & $<1$ \\
\hline & $10 / 16 / 89$ & $<10$ & $<1$ & $<1$ & $14.0 \pm 1.4$ & $190 \pm 20$ & $<1$ & -- & $<1$ & $<1$ & $<1$ \\
\hline \multirow[t]{2}{*}{$S F-18$} & $10 / 05 / 89$ & $<10$ & $<1$ & $<1$ & $10.9 \pm 1.1$ & $200 \pm 20$ & $<1$ & $\cdots$ & $<1$ & $<1$ & $<1$ \\
\hline & $10 / 16 / 89$ & $<10$ & $<1$ & $<1$ & $10.2 \pm 1.0$ & $230 \pm 20$ & $<1$ & -- & $<1$ & $<1$ & $<1$ \\
\hline \multirow[t]{6}{*}{$S F-19$} & $8 / 21 / 88$ & $100 \pm 10$ & $<1$ & $<1$ & $10.6 \pm 1.1$ & $500 \pm 50$ & $<1$ & $64.9 \pm 6.5$ & $<1$ & $<1$ & $<1$ \\
\hline & $9 / 15 / 88$ & $<10$ & $<1$ & $<1$ & $4.5 \pm 0.5$ & $72 \pm 14$ & $<1$ & $18.0 \pm 1.1$ & $<1$ & $<1$ & $<1$ \\
\hline & $9 / 25 / 88$ & $<10$ & $<1$ & $<1$ & $8.2 \pm 0.8$ & $132 \pm 26$ & $<1$ & $33.2 \pm 2.0$ & $<1$ & $<1$ & $<1$ \\
\hline & $1 / 12 / 89$ & $<10$ & $<1$ & $<1$ & $0.5 \pm 0.1$ & $<1$ & $<1$ & $3.2 \pm 0.3$ & $<1$ & $<1$ & $<1$ \\
\hline & $8 / 15 / 89$ & $<10$ & $<1$ & $<1$ & $8.0 \pm 0.8$ & $180 \pm 30$ & $<1$ & $\cdots$ & $<1$ & $<1$ & $<1$ \\
\hline & $10 / 16 / 89$ & $<10$ & $<1$ & $<1$ & $6.9 \pm 0.7$ & $150 \pm 25$ & $<1$ & --- & $<1$ & $<1$ & $<1$ \\
\hline
\end{tabular}




\begin{tabular}{|c|c|c|c|c|c|c|c|c|c|c|c|}
\hline $\begin{array}{l}\text { Site } \\
\text { No. }\end{array}$ & $\begin{array}{c}\text { Collection } \\
\text { Date }\end{array}$ & $\begin{array}{c}T i \\
(u g / L)\end{array}$ & $\begin{array}{c}T l \\
(u g / L)\end{array}$ & $\begin{array}{c}\mathrm{Tm} \\
(\mathrm{ug} / \mathrm{L})\end{array}$ & $\begin{array}{c}U \\
(u g / L)\end{array}$ & $\begin{array}{c}V \\
(u g / L)\end{array}$ & $\begin{array}{c}W \\
(u g / L)\end{array}$ & $\begin{array}{c}Y \\
(u g / L)\end{array}$ & $\begin{array}{c}Y b \\
(u g / L)\end{array}$ & $\begin{array}{c}2 n \\
(u g / L)\end{array}$ & $\begin{array}{c}2 r \\
(u g / L)\end{array}$ \\
\hline \multirow[t]{2}{*}{$M V-5$} & $8 / 15 / 89$ & $<10$ & $<1$ & $<1$ & $<1$ & $<1$ & $<1$ & $<1$ & $<1$ & $<1$ & $<1$ \\
\hline & $10 / 16 / 89$ & $<10$ & $<1$ & $<1$ & $<1$ & $<1$ & $<1$ & $<1$ & $<1$ & $<1$ & $<1$ \\
\hline \multirow[t]{3}{*}{ MV- 11} & $9 / 15 / 88$ & $<10$ & $<1$ & $<1$ & $<1$ & $<1$ & $<1$ & $<1$ & $<1$ & $<1$ & $<1$ \\
\hline & $8 / 15 / 89$ & $<10$ & $<1$ & $<1$ & $<1$ & $<1$ & $<1$ & $<1$ & $<1$ & $<1$ & $<1$ \\
\hline & $10 / 16 / 89$ & $<10$ & $<1$ & $<1$ & $<1$ & $<1$ & $<1$ & $<1$ & $<1$ & $<1$ & $<1$ \\
\hline \multirow[t]{3}{*}{$M V-13$} & $9 / 15 / 88$ & $<10$ & $<1$ & $<1$ & $<1$ & $<1$ & $<1$ & $<1$ & $<1$ & $<1$ & $<1$ \\
\hline & $9 / 13 / 89$ & $<10$ & $<1$ & $<1$ & $<1$ & $<1$ & $<1$ & $<1$ & $<1$ & $<1$ & $<1$ \\
\hline & $10 / 16 / 89$ & $<10$ & $<1$ & $<1$ & $<1$ & $<1$ & $<1$ & $<1$ & $<1$ & $<1$ & $<1$ \\
\hline \multirow[t]{2}{*}{$M V-14 A$} & $8 / 15 / 89$ & $<10$ & $<1$ & $<1$ & $<1$ & $<1$ & $<1$ & $<1$ & $<1$ & $<1$ & $<1$ \\
\hline & $10 / 16 / 89$ & $<10$ & $<1$ & $<1$ & $<1$ & $<1$ & $<1$ & $<1$ & $=1$ & $<1$ & $<1$ \\
\hline \multirow[t]{3}{*}{$M V-14 B$} & $9 / 13 / 89$ & $<10$ & $<1$ & $<1$ & $<1$ & $<1$ & $<1$ & $<1$ & $<1$ & $<1$ & $<1$ \\
\hline & $10 / 5 / 89$ & $<10$ & $<1$ & $<1$ & $<1$ & $<1$ & $<1$ & $<1$ & $<1$ & $<1$ & $<1$ \\
\hline & $10 / 16 / 89$ & $<10$ & $<1$ & $<1$ & $<1$ & $<1$ & $<1$ & $<1$ & $<1$ & $<1$ & $<1$ \\
\hline$M V-20$ & $8 / 15 / 89$ & $<10$ & $<1$ & $<1$ & $<1$ & $<1$ & $<1$ & $<1$ & $<1$ & $<1$ & $<1$ \\
\hline \multirow[t]{4}{*}{$M V-25$} & $9 / 15 / 88$ & $<10$ & $<1$ & $<1$ & $<1$ & $<1$ & $<1$ & $<1$ & $<1$ & $<1$ & $<1$ \\
\hline & $8 / 15 / 89$ & $<10$ & $<1$ & $<1$ & $<1$ & $<1$ & $<1$ & $<1$ & $<1$ & $<1$ & $<1$ \\
\hline & $9 / 13 / 89$ & $<10$ & $<1$ & $<1$ & $<1$ & $<1$ & $<1$ & $<1$ & $<1$ & $=1$ & $<1$ \\
\hline & $10 / 16 / 89$ & $<10$ & $<1$ & $<1$ & $<1$ & $<1$ & $<1$ & $<1$ & $<1$ & $<1$ & $<1$ \\
\hline \multirow[t]{3}{*}{$M V-35$} & $9 / 15 / 88$ & $<10$ & $<1$ & $<1$ & $<1$ & $<1$ & $<1$ & $<1$ & $<1$ & $<1$ & $<1$ \\
\hline & $8 / 15 / 89$ & $<10$ & $<1$ & $<1$ & $<1$ & $<1$ & $<1$ & $<1$ & $<1$ & $<1$ & $<1$ \\
\hline & $10 / 16 / 89$ & $<10$ & $<1$ & $<1$ & $<1$ & $<1$ & $<1$ & $<1$ & $<1$ & $<1$ & $<1$ \\
\hline \multirow[t]{2}{*}{$S F-2$} & $10 / 5 / 89$ & $<10$ & $<1$ & $<1$ & $<1$ & $<1$ & $<1$ & $<1$ & $<1$ & $<1$ & $<1$ \\
\hline & $10 / 16 / 89$ & $<10$ & $<1$ & $<1$ & $<1$ & $<1$ & $<1$ & $<1$ & $<1$ & $<1$ & $<1$ \\
\hline \multirow[t]{7}{*}{$S F-8$} & $8 / 21 / 88$ & $<10$ & $<1$ & $<1$ & $<1$ & $<1$ & $<1$ & $<1$ & $<1$ & $100 \pm 10$ & $<1$ \\
\hline & $9 / 15 / 88$ & $<10$ & $<1$ & $<1$ & $<1$ & $<1$ & $<1$ & $<1$ & $<1$ & $<1$ & $<1$ \\
\hline & $9 / 25 / 88$ & $<10$ & $<1$ & $<1$ & $<1$ & $<1$ & $<1$ & $<1$ & $<1$ & $<1$ & $<1$ \\
\hline & $1 / 12 / 89$ & $<10$ & $<1$ & $<1$ & $<1$ & $<1$ & $8 \pm 2$ & $<1$ & $<1$ & $<1$ & $<1$ \\
\hline & $8 / 15 / 89$ & $<10$ & $<1$ & $<1$ & $<1$ & $<1$ & $<1$ & $<1$ & $<1$ & $<1$ & $<1$ \\
\hline & $10 / 05 / 89$ & $<10$ & $<1$ & $<1$ & $<1$ & $<1$ & $<1$ & $<1$ & $<1$ & $<1$ & $<1$ \\
\hline & $10 / 16 / 89$ & $<10$ & $<1$ & $<1$ & $<1$ & $<1$ & $<1$ & $<1$ & $<1$ & $<1$ & $<1$ \\
\hline
\end{tabular}




\begin{tabular}{|c|c|c|c|c|c|c|c|c|c|c|c|}
\hline $\begin{array}{l}\text { Site } \\
\text { No. }\end{array}$ & $\begin{array}{c}\text { Collection } \\
\text { Date }\end{array}$ & $\begin{array}{c}T i \\
(u g / L)\end{array}$ & $\begin{array}{c}T l \\
(u g / L)\end{array}$ & $\begin{array}{c}\mathrm{Tm} \\
(\mathrm{ug} / \mathrm{L})\end{array}$ & $\begin{array}{c}U \\
(u g / L)\end{array}$ & $\begin{array}{c}V \\
(u g / L)\end{array}$ & $\begin{array}{c}W \\
(u g i l)\end{array}$ & $\begin{array}{c}Y \\
(u g / L)\end{array}$ & $\begin{array}{l}Y b \\
(u g / L)\end{array}$ & $\begin{array}{c}\mathrm{Zn} \\
(u g / L)\end{array}$ & $\begin{array}{c}2 r \\
(u g / L)\end{array}$ \\
\hline \multirow[t]{2}{*}{$S F-9 A$} & $10 / 05 / 89$ & $<10$ & $<1$ & $<1$ & $<1$ & $<1$ & $<1$ & $<1$ & $<1$ & $<1$ & $<1$ \\
\hline & $10 / 16 / 89$ & $<10$ & $<1$ & $<1$ & $<1$ & $<1$ & $<1$ & $<1$ & $<1$ & $<1$ & $<1$ \\
\hline$S F-9 B$ & $7 / 28 / 88$ & $100 \pm 10$ & $<1$ & $<1$ & $<1$ & $10 \pm 1$ & $<1$ & $<1$ & $<1$ & $<1$ & $<1$ \\
\hline \multirow[t]{8}{*}{$S F-10 B$} & $7 / 28 / 88$ & $100 \pm 10$ & $<1$ & $<1$ & $10 \pm 1$ & $10 \pm 1$ & $<1$ & $<1$ & $<1$ & $<1$ & $<1$ \\
\hline & $8 / 21 / 88$ & $<10$ & $<1$ & $<1$ & $10 \pm 1$ & $<1$ & $<1$ & $<1$ & $<1$ & $100 \pm 10$ & $<1$ \\
\hline & $9 / 15 / 88$ & $<10$ & $<1$ & $<1$ & $<1$ & $<1$ & $<1$ & $<1$ & $<1$ & $<1$ & $<1$ \\
\hline & $9 / 25 / 88$ & $<10$ & $<1$ & $<1$ & $<1$ & $<1$ & $<1$ & $<1$ & $<1$ & $<1$ & $<1$ \\
\hline & $1 / 12 / 89$ & $<10$ & $<1$ & $<1$ & $<1$ & $<1$ & $5 \pm 1$ & $<1$ & $<1$ & $<1$ & $<1$ \\
\hline & $8 / 15 / 89$ & $<10$ & $<1$ & $<1$ & $<1$ & $<1$ & $<1$ & $<1$ & $<1$ & $<1$ & $<1$ \\
\hline & $10 / 05 / 89$ & $<10$ & $<1$ & $<1$ & $<1$ & $<1$ & $<1$ & $<1$ & $<1$ & $<1$ & $<1$ \\
\hline & $10 / 16 / 89$ & $<10$ & $<1$ & $<1$ & $<1$ & $<1$ & $<1$ & $<1$ & $<1$ & $<1$ & $<1$ \\
\hline \multirow[t]{2}{*}{$S F-12 A$} & $7 / 28 / 88$ & $100 \pm 10$ & $<1$ & $<1$ & $10 \pm 1$ & $10 \pm 1$ & $<1$ & $<1$ & $<1$ & $10 \pm 1$ & $<1$ \\
\hline & $9 / 25 / 88$ & $<10$ & $<1$ & $<1$ & $<1$ & $<1$ & $<1$ & $<1$ & $<1$ & $<1$ & $<1$ \\
\hline \multirow[t]{8}{*}{$S F-12 B$} & $7 / 28 / 88$ & $100 \pm 10$ & $<1$ & $<1$ & $<1$ & $10 \pm 1$ & $<1$ & $<1$ & $<1$ & $<1$ & $<1$ \\
\hline & $8 / 21 / 88$ & $<10$ & $<1$ & $<1$ & $5 \pm 1$ & $<1$ & $<1$ & $<1$ & $<1$ & $100 \pm 10$ & $<1$ \\
\hline & $9 / 15 / 88$ & $<10$ & $<1$ & $<1$ & $<1$ & $<1$ & $<1$ & $<1$ & $<1$ & $<1$ & $<1$ \\
\hline & $9 / 25 / 88$ & $<10$ & $<1$ & $<1$ & $<1$ & $<1$ & $<1$ & $<1$ & $<1$ & $<1$ & $<1$ \\
\hline & $1 / 12 / 89$ & $<10$ & $<1$ & $<1$ & $<1$ & $<1$ & $4 \pm 1$ & $<1$ & $<1$ & $<1$ & $<1$ \\
\hline & $8 / 15 / 89$ & $<10$ & $<1$ & $<1$ & $<1$ & $<1$ & $<1$ & $<1$ & $<1$ & $<1$ & $<1$ \\
\hline & $10 / 05 / 89$ & $<10$ & $<1$ & $<1$ & $<1$ & $<1$ & $<1$ & $<1$ & $<1$ & $<1$ & $<1$ \\
\hline & $10 / 16 / 89$ & $<10$ & $<1$ & $<1$ & $<1$ & $<1$ & $<1$ & $<1$ & $<1$ & $<1$ & $<1$ \\
\hline \multirow[t]{7}{*}{ SF -13} & $7 / 28 / 88$ & $100 \pm 10$ & $<1$ & $<1$ & $<1$ & $10 \pm 1$ & $<1$ & $<1$ & $<1$ & $<1$ & $<1$ \\
\hline & $8 / 21 / 88$ & $<10$ & $<1$ & $<1$ & $5 \pm 1$ & $<1$ & $<1$ & $<1$ & $<1$ & $100 \pm 10$ & $<1$ \\
\hline & $9 / 15 / 88$ & $<10$ & $<1$ & $<1$ & $<1$ & $<1$ & $<1$ & $<i$ & $<1$ & $<1$ & $<1$ \\
\hline & $1 / 12 / 89$ & $<10$ & $<1$ & $<1$ & $<1$ & $<1$ & $7 \pm 2$ & $<1$ & $<1$ & $5 \pm 1$ & $<1$ \\
\hline & $8 / 15 / 89$ & $<10$ & $<1$ & $<1$ & $<1$ & $<1$ & $<1$ & $<1$ & $<1$ & $<1$ & $<1$ \\
\hline & $10 / 05 / 89$ & $<10$ & $<1$ & $<1$ & $<1$ & $<1$ & $<1$ & $<1$ & $<1$ & $<1$ & $<1$ \\
\hline & $10 / 16 / 89$ & $<10$ & $<1$ & $<1$ & $<1$ & $<1$ & $<1$ & $<1$ & $<1$ & $<1$ & $<1$ \\
\hline \multirow[t]{2}{*}{$S F-18$} & $10 / 05 / 89$ & $<10$ & $<1$ & $<1$ & $<1$ & $<1$ & $<1$ & $<1$ & $<1$ & $<1$ & $<1$ \\
\hline & $10 / 16 / 89$ & $<10$ & $<1$ & $<1$ & $<1$ & $<1$ & $<1$ & $<1$ & $<1$ & $<1$ & $<1$ \\
\hline \multirow[t]{6}{*}{$S F-19$} & $8 / 21 / 88$ & $<10$ & $<1$ & $<1$ & $10 \pm 1$ & $<1$ & $<1$ & $<1$ & $<1$ & $100 \pm 10$ & $<1$ \\
\hline & $9 / 15 / 88$ & $<10$ & $<1$ & $<1$ & $<1$ & $<1$ & $<1$ & $<1$ & $<1$ & $<1$ & $<1$ \\
\hline & $9 / 25 / 88$ & $<10$ & $<1$ & $<1$ & $<1$ & $<1$ & $<1$ & $<1$ & $<1$ & $<1$ & $<1$ \\
\hline & $1 / 12 / 89$ & $<10$ & $<1$ & $<1$ & $<1$ & $<1$ & $2 \pm 1$ & $<1$ & $<1$ & $<1$ & $<1$ \\
\hline & $8 / 15 / 89$ & $<10$ & $<1$ & $<1$ & $<1$ & $<1$ & $<1$ & $<1$ & $<1$ & $<1$ & $<1$ \\
\hline & $10 / 16 / 89$ & $<10$ & $<1$ & $<1$ & $<1$ & $<1$ & $<1$ & $<1$ & $<1$ & $<1$ & $<1$ \\
\hline
\end{tabular}




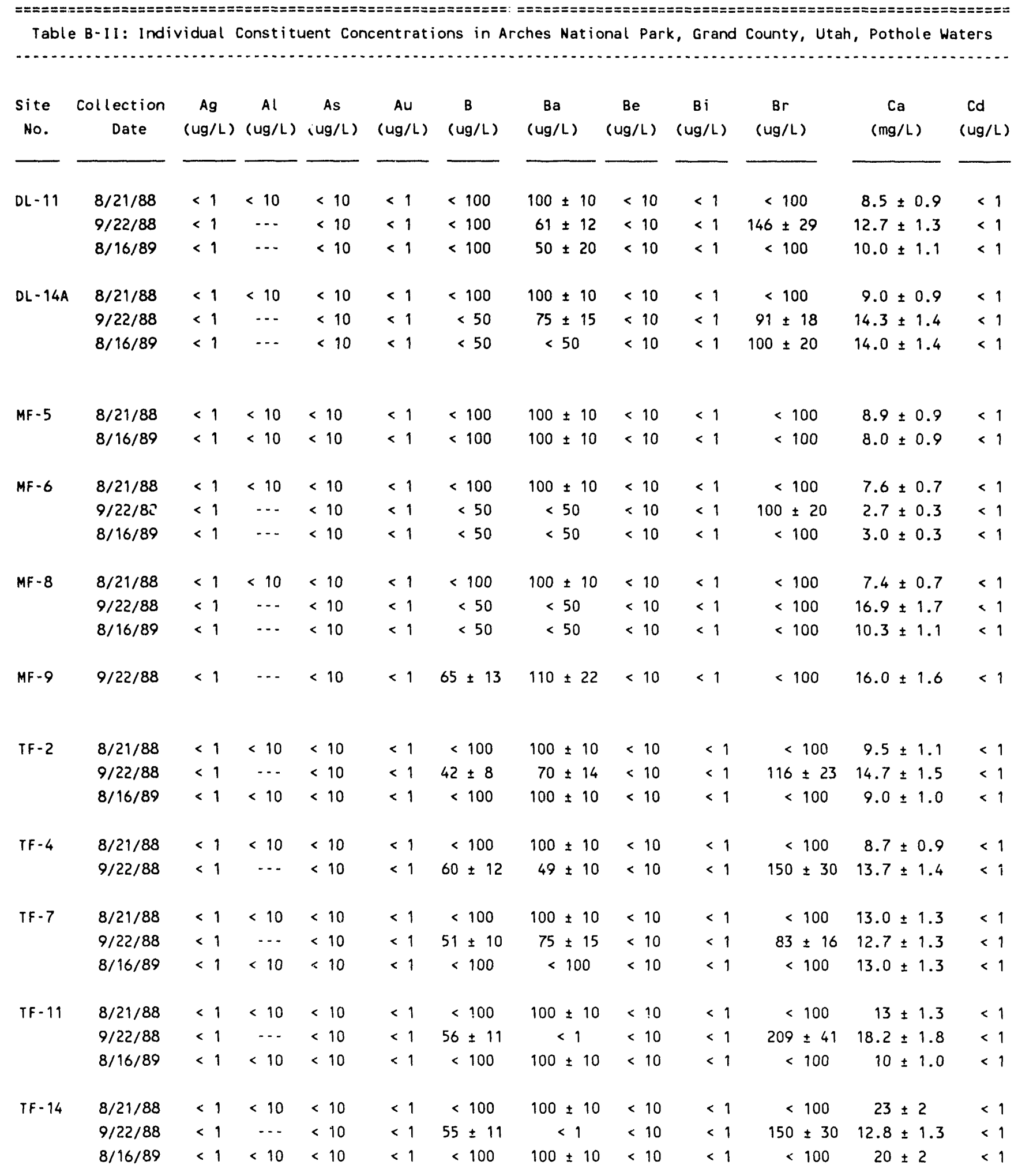






\footnotetext{
* Cond. $=$ Conductivity
} 


\begin{tabular}{|c|c|c|c|c|c|c|c|c|c|c|c|c|c|}
\hline $\begin{array}{l}\text { Site } \\
\text { No. }\end{array}$ & $\begin{array}{c}\text { Collection } \\
\text { Date }\end{array}$ & $\begin{array}{c}G a \\
(u g / L)\end{array}$ & $\begin{array}{c}\text { Gd } \\
(u g / L)\end{array}$ & $\begin{array}{c}\mathrm{Ge} \\
(u g / L)\end{array}$ & $\begin{array}{c}\text { Hf } \\
(u g / L)\end{array}$ & $\begin{array}{c}\mathrm{Hg} \\
(u g / L)\end{array}$ & $\begin{array}{c}\text { Ho } \\
\text { (ug/L) }\end{array}$ & $\begin{array}{c}1 \\
(u g / L)\end{array}$ & $\begin{array}{c}\text { In } \\
(u g / L)\end{array}$ & $\begin{array}{c}\text { Ir } \\
(u g / L)\end{array}$ & $\begin{array}{c}K \\
(m g / L)\end{array}$ & $\begin{array}{c}\text { La } \\
(u g / L)\end{array}$ & $\begin{array}{c}L i \\
(u g / L)\end{array}$ \\
\hline \multirow[t]{3}{*}{$D L-11$} & $8 / 21 / 88$ & $<1$ & $<1$ & $<1$ & $<1$ & $<1$ & $<1$ & $10 \pm 1$ & $<1$ & $<1$ & $1.9 \pm 0.2$ & $<1$ & $<10$ \\
\hline & $9 / 22 / 88$ & $<1$ & $<1$ & $<1$ & $<1$ & $<1$ & $<1$ & $<1$ & $<1$ & $<1$ & $1.3 \pm 0.1$ & $<1$ & $<10$ \\
\hline & $8 / 16 / 89$ & $<1$ & $<1$ & $<1$ & $<1$ & $<1$ & $<1$ & $<1$ & $<1$ & $<1$ & $1.6 \pm 0.2$ & $<1$ & $<10$ \\
\hline \multirow{3}{*}{$D L-14 A$} & $8 / 21 / 88$ & $<1$ & $<1$ & $<1$ & $<1$ & $<1$ & $<1$ & $10 \pm 1$ & $<1$ & $<1$ & $1.6 \pm 0.2$ & $<1$ & $<10$ \\
\hline & $9 / 22 / 88$ & $<1$ & $<1$ & $<1$ & $<1$ & $<1$ & $<1$ & $<1$ & $<1$ & $<1$ & $1.0 \pm 0.1$ & $<1$ & $<10$ \\
\hline & $8 / 16 / 89$ & $<1$ & $<1$ & $<1$ & $<1$ & $<1$ & $<1$ & $<1$ & $<1$ & $<1$ & $1.3 \pm 0.1$ & $<1$ & $<10$ \\
\hline \multirow[t]{2}{*}{$M F-5$} & $8 / 21 / 88$ & $<1$ & $<1$ & $<1$ & $<1$ & $<1$ & $<1$ & $10 \pm 1$ & $<1$ & $<1$ & $1.9 \pm 0.2$ & $<1$ & $<10$ \\
\hline & $8 / 16 / 89$ & $<1$ & $<1$ & $<1$ & $<1$ & $<1$ & $<1$ & $<10$ & $<1$ & $<1$ & $1.7 \pm 0.2$ & $<1$ & $<10$ \\
\hline \multirow[t]{3}{*}{ MF - 6} & $8 / 21 / 88$ & $<1$ & $<1$ & $<1$ & $<1$ & $<1$ & $<1$ & $10 \pm 1$ & $<1$ & $<1$ & $1.4 \pm 0.1$ & $<1$ & $<10$ \\
\hline & $9 / 22 / 88$ & $<1$ & $<1$ & $<1$ & $<1$ & $<1$ & $<1$ & $<1$ & $<1$ & $<1$ & $1.2 \pm 0.1$ & $<1$ & $<10$ \\
\hline & $8 / 16 / 89$ & $<1$ & $<1$ & $<1$ & $<1$ & $<1$ & $<1$ & $<1$ & $<1$ & $<1$ & $1.1 \pm 0.1$ & $<1$ & $<10$ \\
\hline \multirow[t]{3}{*}{$M F-8$} & $8 / 21 / 88$ & $<1$ & $<1$ & $<1$ & $<1$ & $<1$ & $<1$ & $10 \pm 1$ & $<1$ & $<1$ & $4.4 \pm 0.4$ & $<1$ & $<10$ \\
\hline & $9 / 22 / 88$ & $<1$ & $<1$ & $<1$ & $<1$ & $<1$ & $<1$ & $<1$ & $<1$ & $<1$ & $1.7 \pm 0.2$ & $<1$ & $<10$ \\
\hline & $8 / 16 / 89$ & $<1$ & $<1$ & $<1$ & $<1$ & $<1$ & $<1$ & $<1$ & $<1$ & $<1$ & $1.4 \pm 0.1$ & $<1$ & $<10$ \\
\hline MF-9 & $9 / 22 / 88$ & $<1$ & $<1$ & $<1$ & $<1$ & $<1$ & $<1$ & $<1$ & $<1$ & $<1$ & $1.9 \pm 0.2$ & $<1$ & $<10$ \\
\hline \multirow[t]{3}{*}{$T F-2$} & $8 / 21 / 88$ & $<1$ & $<1$ & $<1$ & $<1$ & $<1$ & $<1$ & $10 \pm 1$ & $<1$ & $<1$ & $1.5 \pm 0.2$ & $<1$ & $<10$ \\
\hline & $9 / 22 / 88$ & $<1$ & $<1$ & $<1$ & $<1$ & $<1$ & $<1$ & $<1$ & $<1$ & $<1$ & $1.0 \pm 0.1$ & $<1$ & $<10$ \\
\hline & $8 / 16 / 89$ & $<1$ & $<1$ & $<1$ & $<1$ & $<1$ & $<1$ & $<1$ & $<1$ & $<1$ & $1.3 \pm 0.1$ & $<1$ & $<10$ \\
\hline \multirow[t]{2}{*}{$T F-4$} & $8 / 21 / 88$ & $<1$ & $<1$ & $<1$ & $<1$ & $<1$ & $<1$ & $10 \pm 1$ & $<1$ & $<1$ & $2.0 \pm 0.2$ & $<1$ & $<10$ \\
\hline & $9 / 22 / 88$ & $<1$ & $<1$ & $<1$ & $<1$ & $<1$ & $<1$ & $<1$ & $<1$ & $<1$ & $1.2 \pm 0.1$ & $<1$ & $<10$ \\
\hline \multirow[t]{3}{*}{$T F-7$} & $8 / 21 / 88$ & $<1$ & $<1$ & $<1$ & $<1$ & $<1$ & $<1$ & $10 \pm 1$ & $<1$ & $<1$ & $2.2 \pm 0.2$ & $<1$ & $<10$ \\
\hline & $9 / 22 / 88$ & $<1$ & $<1$ & $<1$ & $<1$ & $<1$ & $<1$ & $<1$ & $<1$ & $<1$ & $1.3 \pm 0.1$ & $<1$ & $<10$ \\
\hline & $8 / 16 / 89$ & $<1$ & $<1$ & $<1$ & $<1$ & $<1$ & $<1$ & $<1$ & $<1$ & $<1$ & $1.0 \pm 0.1$ & $<1$ & $<10$ \\
\hline \multirow[t]{3}{*}{$T F-11$} & $8 / 21 / 88$ & $<1$ & $<1$ & $<1$ & $<1$ & $<1$ & $<1$ & $10 \pm 1$ & $<1$ & $<1$ & $1.9 \pm 0.2$ & $<1$ & $<10$ \\
\hline & $9 / 22 / 88$ & $<1$ & $<1$ & $<1$ & $<1$ & $<1$ & $<1$ & $<1$ & $<1$ & $<1$ & $1.5 \pm 0.2$ & $<1$ & $<10$ \\
\hline & $8 / 16 / 89$ & $<1$ & $<1$ & $<1$ & $<1$ & $<1$ & $<1$ & $<1$ & $<1$ & $<1$ & $1.1 \pm 0.1$ & $<1$ & $<10$ \\
\hline \multirow[t]{3}{*}{$T F-14$} & $8 / 21 / 88$ & $<1$ & $<1$ & $<1$ & $<1$ & $<1$ & $<1$ & $10 \pm 1$ & $<1$ & $<1$ & $1.9 \pm 0.2$ & $<1$ & $<10$ \\
\hline & $9 / 22 / 88$ & $<1$ & $<1$ & $<1$ & $<1$ & $<1$ & $<1$ & $<1$ & $<1$ & $<1$ & $1.1 \pm 0.1$ & $<1$ & $<10$ \\
\hline & $8 / 16 / 89$ & $<1$ & $<1$ & $<1$ & $<1$ & $<1$ & $<1$ & $<1$ & $<1$ & $<1$ & $1.0 \pm 0.1$ & $<1$ & $<10$ \\
\hline
\end{tabular}




\begin{tabular}{|c|c|c|c|c|c|c|c|c|c|c|c|}
\hline $\begin{array}{l}\text { Site } \\
\text { No. }\end{array}$ & $\begin{array}{c}\text { Collection } \\
\text { Date }\end{array}$ & $\begin{array}{c}\text { Lu } \\
(u g / L)\end{array}$ & $\begin{array}{c}M g \\
(m g / L)\end{array}$ & $\begin{array}{c}M n \\
(u g / L)\end{array}$ & $\begin{array}{c}\text { Mo } \\
\text { (ug/L) }\end{array}$ & $\begin{array}{c}\mathrm{Na} \\
(m g / L)\end{array}$ & $\begin{array}{c}N b \\
(u g / L)\end{array}$ & $\begin{array}{c}\text { Nd } \\
(u g / L)\end{array}$ & $\begin{array}{c}\mathrm{Ni} \\
(u g / L)\end{array}$ & $\begin{array}{l}\mathrm{NO}_{3}-\mathrm{N} \\
(\mathrm{mg} / \mathrm{L})\end{array}$ & $\begin{array}{c}\text { Os } \\
(u g / L)\end{array}$ \\
\hline \multirow[t]{3}{*}{$D L-11$} & $8 / 21 / 88$ & $<1$ & $1.0 \pm 0.1$ & $500 \pm 50$ & $<1$ & $1.0 \pm 0.1$ & $<1$ & $<1$ & $100 \pm 10$ & $1.0 \pm 0.1$ & $<1$ \\
\hline & $9 / 22 / 88$ & $<1$ & $1.3 \pm 0.1$ & $70 \pm 14$ & $<1$ & $1.3 \pm 0.1$ & $<1$ & $<1$ & $<1$ & $<0.2$ & $<1$ \\
\hline & $8 / 16 / 89$ & $<1$ & $1.3 \pm 0.1$ & $80 \pm 10$ & $<1$ & $1.1 \pm 0.1$ & $<1$ & $<1$ & $<1$ & $<0.2$ & $<1$ \\
\hline \multirow[t]{3}{*}{$D L-14 A$} & $8 / 21 / 88$ & $<10$ & $0.93 \pm 0.09$ & $500 \pm 50$ & $<1$ & $1.0 \pm 0.1$ & $<1$ & $<1$ & $50 \pm 5$ & $0.8 \pm 0.1$ & $<1$ \\
\hline & $9 / 22 / 88$ & $<1$ & $1.2 \pm 0.1$ & $95 \pm 19$ & $<1$ & $1.1 \pm 0.1$ & $<1$ & $<1$ & $<50$ & $<0.2$ & $<1$ \\
\hline & $8 / 16 / 89$ & $<1$ & $1.0 \pm 0.1$ & $110 \pm 20$ & $<1$ & $1.4 \pm 0.1$ & $<1$ & $<1$ & $<50$ & $<0.2$ & $<1$ \\
\hline \multirow[t]{2}{*}{$M F-5$} & $8 / 21 / 88$ & $<10$ & $0.99 \pm 0.1$ & $100 \pm 10$ & $<1$ & $0.9 \pm 0.1$ & $<1$ & $<1$ & $100 \pm 10$ & $0.6 \pm 0.1$ & $<1$ \\
\hline & $8 / 16 / 89$ & $<1$ & $1.0 \pm 0.1$ & $100 \pm 10$ & $<1$ & $1.5 \pm 0.1$ & $<1$ & $<1$ & $<50$ & $0.5 \pm 0.1$ & $<1$ \\
\hline \multirow[t]{3}{*}{ MF- 6} & $8 / 21 / 88$ & $<10$ & $0.66 \pm 0.07$ & $100 \pm 10$ & $<1$ & $0.4 \pm 0.1$ & $<1$ & $<1$ & $100 \pm 10$ & $0.8 \pm 0.1$ & $<1$ \\
\hline & $9 / 22 / 88$ & $<1$ & $1.0 \pm 0.1$ & $<50$ & $<1$ & $1.2 \pm 0.1$ & $<1$ & $<1$ & $79 \pm 16$ & $<0.2$ & $<1$ \\
\hline & $8 / 16 / 89$ & $<1$ & $0.9 \pm 0.1$ & $<50$ & $<1$ & $1.1 \pm 0.1$ & $<1$ & $<1$ & $60 \pm 10$ & $<0.2$ & $<1$ \\
\hline \multirow[t]{3}{*}{$M F-8$} & $8 / 21 / 88$ & $<1$ & $1.7 \pm 0.2$ & $100 \pm 10$ & $<1$ & $1.4 \pm 0.1$ & $<1$ & $<1$ & $100 \pm 10$ & $1.1 \pm 0.1$ & $<1$ \\
\hline & $9 / 22 / 88$ & $<1$ & $1.3 \pm 0.1$ & $<50$ & $<1$ & $1.6 \pm 0.2$ & $<1$ & $<1$ & $<50$ & $<0.2$ & $<1$ \\
\hline & $8 / 16 / 89$ & $<1$ & $1.0 \pm 0.1$ & $<50$ & $<1$ & $1.4 \pm 0.1$ & $<1$ & $<1$ & $<50$ & $<0.2$ & $<1$ \\
\hline$M F-9$ & $9 / 22 / 88$ & $<1$ & $1.6 \pm 0.2$ & $100 \pm 20$ & $<1$ & $1.2 \pm 0.1$ & $<1$ & $<1$ & $<50$ & $<0.2$ & $<1$ \\
\hline \multirow[t]{3}{*}{ TF -2} & $8 / 21 / 88$ & $<1$ & $0.9 \pm 0.1$ & $100 \pm 10$ & $<1$ & $1.0 \pm 0.1$ & $<1$ & $<1$ & $100 \pm 10$ & $1.5 \pm 0.2$ & $<1$ \\
\hline & $9 / 22 / 88$ & $<1$ & $1.2 \pm 0.1$ & $87 \pm 17$ & $<1$ & $1.3 \pm 0.1$ & $<1$ & $<1$ & $<1$ & $0.5 \pm 0.1$ & $<1$ \\
\hline & $8 / 16 / 89$ & $<1$ & $1.0 \pm 0.1$ & $70 \pm 15$ & $<1$ & $1.1 \pm 0.1$ & $<1$ & $<1$ & $<1$ & $0.8 \pm 0.1$ & $<1$ \\
\hline \multirow[t]{2}{*}{ TF -4} & $8 / 21 / 88$ & $<1$ & $0.97 \pm 0.1$ & $100 \pm 10$ & $<1$ & $1.0 \pm 0.1$ & $<1$ & $<1$ & $1000 \pm 100$ & $1.5 \pm 0.2$ & $<1$ \\
\hline & $9 / 22 / 88$ & $<1$ & $1.4 \pm 0.1$ & $48 \pm 10$ & $<1$ & $1.4 \pm 0.1$ & $<1$ & $<1$ & $<1$ & $0.2 \pm 0.1$ & $<1$ \\
\hline \multirow[t]{3}{*}{$T F-7$} & $8 / 21 / 88$ & $<1$ & $1.1 \pm 0.1$ & $500 \pm 50$ & $<1$ & $1.0 \pm 0.1$ & $<1$ & $<1$ & $100 \pm 10$ & $0.6 \pm 0.1$ & $<1$ \\
\hline & $9 / 22 / 88$ & $<1$ & $1.3 \pm 0.1$ & $155 \pm 31$ & $<1$ & $1.1 \pm 0.1$ & $<1$ & $<1$ & $<1$ & $<0.2$ & $<1$ \\
\hline & $8 / 16 / 89$ & $<1$ & $1.0 \pm 0.1$ & $120 \pm 20$ & $<1$ & $1.4 \pm 0.1$ & $<1$ & $<1$ & $<1$ & $<0.2$ & $<1$ \\
\hline \multirow[t]{3}{*}{$T F-11$} & $8 / 21 / 88$ & $<1$ & $1.2 \pm 0.1$ & $100 \pm 10$ & $<1$ & $1.6 \pm 0.2$ & $<1$ & $<1$ & $100 \pm 10$ & $1.2 \pm 0.1$ & $<1$ \\
\hline & $9 / 22 / 88$ & $<1$ & $1.3 \pm 0.1$ & $380 \pm 76$ & $<1$ & $1.8 \pm 0.2$ & $<1$ & $<1$ & $81 \pm 16$ & $<0.2$ & $<1$ \\
\hline & $8 / 16 / 89$ & $<1$ & $1.4 \pm 0.1$ & $300 \pm 50$ & $<1$ & $1.4 \pm 0.1$ & $<1$ & $<1$ & $100 \pm 20$ & $<0.2$ & $<1$ \\
\hline \multirow[t]{3}{*}{ TF -14} & $8 / 21 / 88$ & $<1$ & $1.2 \pm 0.1$ & $500 \pm 50$ & $<1$ & $1.8 \pm 0.2$ & $<1$ & $<1$ & $100 \pm 10$ & $0.7 \pm 0.1$ & $<1$ \\
\hline & $9 / 22 / 88$ & $<1$ & $1.1 \pm 0.1$ & $56 \pm 11$ & $<1$ & $1.4 \pm 0.1$ & $<1$ & $<1$ & $<1$ & $<0.2$ & $<1$ \\
\hline & $8 / 16 / 89$ & $<1$ & $1.4 \pm 0.1$ & $50 \pm 10$ & $<1$ & $1.3 \pm 0.1$ & $<1$ & $<1$ & $<1$ & $0.4 \pm 0.2$ & $<1$ \\
\hline
\end{tabular}




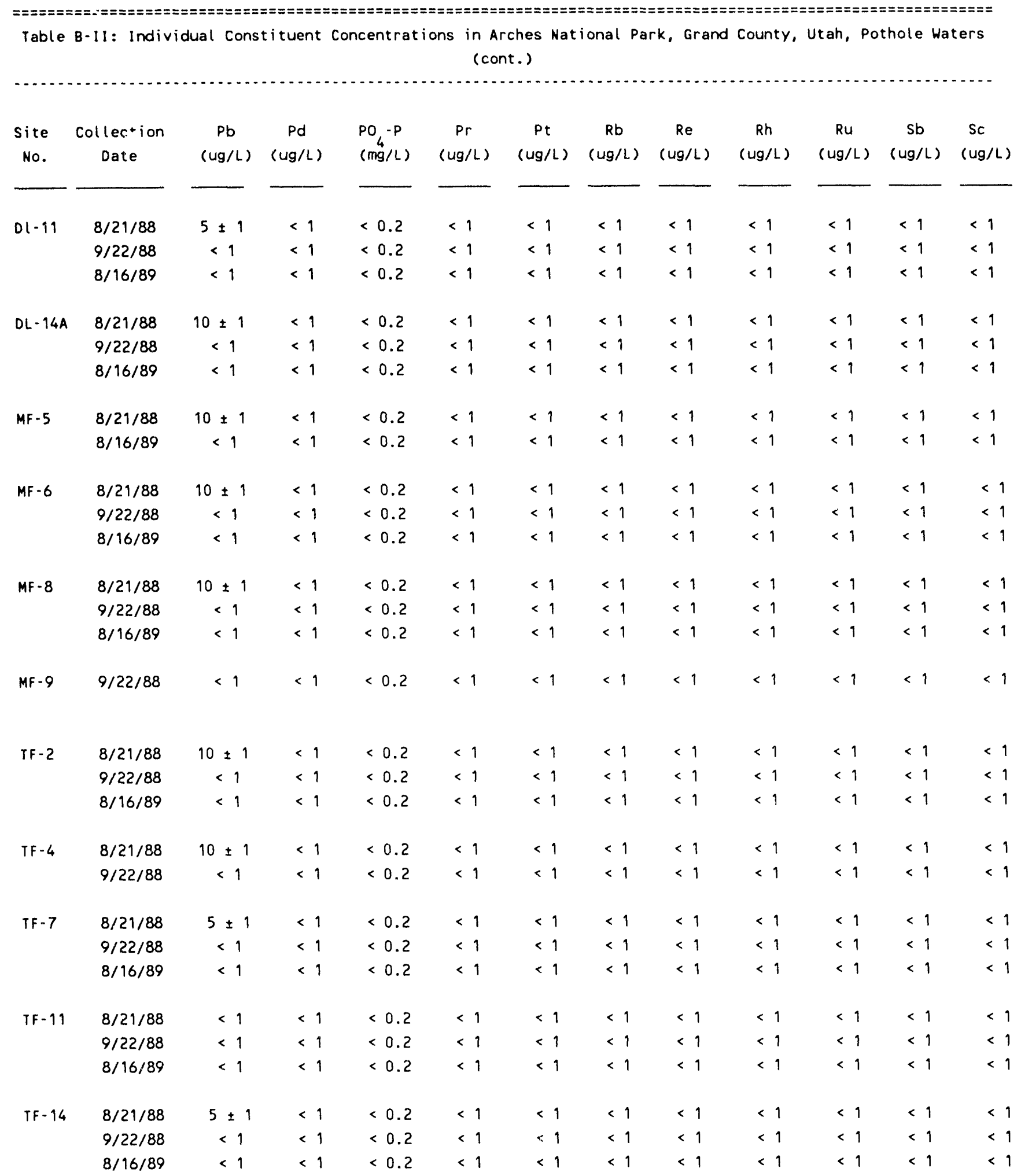




\begin{tabular}{|c|c|c|c|c|c|c|c|c|c|c|c|}
\hline $\begin{array}{c}\text { Site } \\
\text { No. }\end{array}$ & $\begin{array}{c}\text { Collection } \\
\text { Date }\end{array}$ & $\begin{array}{c}\mathrm{Se} \\
(\mathrm{ug} / \mathrm{L})\end{array}$ & $\begin{array}{c}\text { Sm } \\
(u g / L)\end{array}$ & $\begin{array}{c}S n \\
(u g / L)\end{array}$ & $\begin{array}{l}\mathrm{SO}_{4} \\
(\mathrm{mg} / \mathrm{L})\end{array}$ & $\begin{array}{c}S r \\
(u g / L)\end{array}$ & $\begin{array}{c}\mathrm{Ta} \\
(u g / L)\end{array}$ & $\begin{array}{l}\text { T Alk. * } \\
(\mathrm{mg} / \mathrm{L})\end{array}$ & $\begin{array}{c}\text { Tb } \\
(u g / L)\end{array}$ & $\begin{array}{c}\text { Te } \\
(u g / L)\end{array}$ & $\begin{array}{c}\text { Th } \\
\text { (ug/L }\end{array}$ \\
\hline \multirow[t]{3}{*}{$D L-11$} & $8 / 21 / 88$ & $100 \pm 10$ & $<1$ & $<1$ & $11.4 \pm 1.1$ & $500 \pm 50$ & $<1$ & $17.8 \pm 1.8$ & $<1$ & $<1$ & $<1$ \\
\hline & $9 / 22 / 88$ & $<10$ & $<1$ & $<1$ & $16.3 \pm 1.6$ & $154 \pm 31$ & $<1$ & $26.9 \pm 1.6$ & $<1$ & $<1$ & $<1$ \\
\hline & $8 / 16 / 89$ & $<10$ & $<1$ & $<1$ & $14.2 \pm 1.4$ & $300 \pm 30$ & $<1$ & -- & $<1$ & $<1$ & $<1$ \\
\hline \multirow[t]{3}{*}{$D L-14 A$} & $8 / 21 / 88$ & $50 \pm 5$ & $<1$ & $<1$ & $12.2 \pm 1.2$ & $500 \pm 50$ & $<1$ & $19.6 \pm 2$ & $<1$ & $<1$ & $<1$ \\
\hline & $9 / 22 / 88$ & $<10$ & $<1$ & $<1$ & $16.5 \pm 1.7$ & $170 \pm 28$ & $<1$ & $27.8 \pm 1.7$ & $<1$ & $<1$ & $<1$ \\
\hline & $8 / 16 / 89$ & $<10$ & $<1$ & $<1$ & $14.5 \pm 1.4$ & $400 \pm 40$ & $<1$ & $-\cdots$ & $<1$ & $<1$ & $<1$ \\
\hline \multirow[t]{2}{*}{$M F-5$} & $8 / 21 / 88$ & $50 \pm 5$ & $<1$ & $<1$ & $8.7 \pm 0.9$ & $500 \pm 50$ & $<1$ & $23.0 \pm 2.3$ & $<1$ & $<1$ & $<1$ \\
\hline & $8 / 16 / 89$ & $<10$ & $<1$ & $<1$ & $9.7 \pm 0.9$ & $500 \pm 50$ & $<1$ & --- & $<1$ & $<1$ & $<1$ \\
\hline \multirow[t]{3}{*}{$M F-6$} & $8 / 21 / 88$ & $50 \pm 5$ & $<1$ & $<1$ & $7.7 \pm 0.8$ & $500 \pm 50$ & $<1$ & $17.6 \pm 1.7$ & $<1$ & $<1$ & $<1$ \\
\hline & $9 / 22 / 88$ & $<10$ & $<1$ & $<1$ & $.15 .5 \pm 1.6$ & $135 \pm 27$ & $<1$ & $30.4 \pm 1.8$ & $<1$ & $<1$ & $<1$ \\
\hline & $8 / 16 / 89$ & $<10$ & $<1$ & $<1$ & $13.1 \pm 1.3$ & $300 \pm 30$ & $<1$ & $-\infty$ & $<1$ & $<1$ & $<1$ \\
\hline \multirow[t]{3}{*}{$M F-8$} & $8 / 21 / 88$ & $50 \pm 5$ & $<1$ & $<1$ & $8.8 \pm 0.9$ & $500 \pm 50$ & $<1$ & $14.2 \pm 1.4$ & $<1$ & $<1$ & $<1$ \\
\hline & $9 / 22 / 88$ & $<10$ & $<1$ & $<1$ & $24.8 \pm 2.5$ & $<50$ & $<1$ & $25.9 \pm 1.6$ & $<1$ & $<1$ & $<1$ \\
\hline & $8 / 16 / 89$ & $20 \pm 10$ & $<1$ & $<1$ & $10.7 \pm 0.9$ & $500 \pm 50$ & $<1$ & -- & $<1$ & $<1$ & $<1$ \\
\hline$M F-9$ & $9 / 22 / 88$ & $<10$ & $<1$ & $<1$ & $12.9 \pm 1.3$ & $200 \pm 40$ & $<1$ & $37.4 \pm 2.2$ & $<1$ & $<1$ & $<1$ \\
\hline \multirow[t]{3}{*}{$T F-2$} & $8 / 21 / 88$ & $50 \pm 5$ & $=1$ & $<1$ & $11.6 \pm 1.2$ & $500 \pm 50$ & $<1$ & $18.4 \pm 1.8$ & $<1$ & $<1$ & $<1$ \\
\hline & $9 / 22 / 88$ & $<10$ & $<1$ & $<1$ & $21.0 \pm 2.1$ & $190 \pm 38$ & $<1$ & $24.2 \pm 1.4$ & $<1$ & $<1$ & $<1$ \\
\hline & $8 / 16 / 89$ & $<10$ & $<1$ & $<1$ & $20.2 \pm 2.0$ & $140 \pm 20$ & $<1$ & $-\cdots$ & $<1$ & $<1$ & $<1$ \\
\hline \multirow[t]{2}{*}{$T F-4$} & $8 / 21 / 88$ & $50 \pm 5$ & $<1$ & $<1$ & $12.0 \pm 1.2$ & $500 \pm 50$ & $<1$ & $15.6 \pm 1.6$ & $<1$ & $<1$ & $<1$ \\
\hline & $9 / 22 / 88$ & $<10$ & $<1$ & $<1$ & $23.0 \pm 2.3$ & $130 \pm 26$ & $<1$ & $22.6 \pm 1.4$ & $<1$ & $<1$ & $<1$ \\
\hline \multirow[t]{3}{*}{$T F-7$} & $8 / 21 / 88$ & $100 \pm 10$ & $<1$ & $<1$ & $12.8 \pm 1.3$ & $500 \pm 50$ & $<1$ & $28.8 \pm 2.9$ & $<1$ & $<1$ & $<1$ \\
\hline & $9 / 22 / 88$ & $<10$ & $<1$ & $<1$ & $14.4 \pm 1.4$ & $129 \pm 25$ & $<1$ & $27.3 \pm 1.6$ & $<1$ & $<1$ & $<1$ \\
\hline & $8 / 16 / 89$ & $<10$ & $<1$ & $<1$ & $14.0 \pm 1.4$ & $160 \pm 30$ & $<1$ & --- & $<1$ & $<1$ & $<1$ \\
\hline \multirow[t]{3}{*}{$T F-11$} & $8 / 21 / 88$ & $100 \pm 10$ & $<1$ & $<1$ & $18.2 \pm 1.8$ & $500 \pm 50$ & $<1$ & $21.6 \pm 2.2$ & $<1$ & $<1$ & $<1$ \\
\hline & $9 / 22 / 88$ & $<10$ & $<1$ & $<1$ & $26.8 \pm 2.7$ & $193 \pm 38$ & $<1$ & $30.7 \pm 1.8$ & $<1$ & $<1$ & $<1$ \\
\hline & $8 / 16 / 89$ & $<10$ & $<1$ & $<1$ & $19.8 \pm 2.7$ & $170 \pm 25$ & $<1$ & -- & $<1$ & $<1$ & $<1$ \\
\hline \multirow[t]{3}{*}{$T F-14$} & $8 / 21 / 88$ & $100 \pm 10$ & $<1$ & $<1$ & $13.9 \pm 1.4$ & $500 \pm 50$ & $<1$ & $19.4 \pm 1.9$ & $<1$ & $<1$ & $<1$ \\
\hline & $9 / 22 / 88$ & $<10$ & $<1$ & $<1$ & $17.7 \pm 1.8$ & $125 \pm 25$ & $<1$ & $16.2 \pm 1.0$ & $<1$ & $<1$ & $<1$ \\
\hline & $8 / 16 / 89$ & $<10$ & $<1$ & $<1$ & $15.2 \pm 1.5$ & $100 \pm 20$ & $<1$ & --- & $<1$ & $<1$ & $<1$ \\
\hline
\end{tabular}

* TAlk. = Total Alkalinity 


\begin{tabular}{|c|c|c|c|c|c|c|c|c|c|c|c|}
\hline $\begin{array}{l}\text { Site } \\
\text { No. }\end{array}$ & $\begin{array}{c}\text { Collection } \\
\text { Date }\end{array}$ & $\begin{array}{c}T i \\
(u g / L)\end{array}$ & $\begin{array}{c}T l \\
(u g / L)\end{array}$ & $\begin{array}{c}\text { Tm } \\
(u g / L)\end{array}$ & $\begin{array}{c}U \\
(u g / L)\end{array}$ & $\begin{array}{c}V \\
(u g / L)\end{array}$ & $\begin{array}{c}W \\
(u g / L)\end{array}$ & $\begin{array}{c}Y \\
(u g / L)\end{array}$ & $\begin{array}{c}Y b \\
(u g / L)\end{array}$ & $\begin{array}{c}\mathrm{Zn} \\
(\mathrm{ug} / \mathrm{L})\end{array}$ & $\begin{array}{c}2 r \\
(u g / L)\end{array}$ \\
\hline \multirow[t]{3}{*}{$D L-11$} & $8 / 21 / 88$ & $<10$ & $<1$ & $<1$ & $5 \pm 1$ & $<1$ & $<1$ & $<1$ & $<1$ & $100 \pm 10$ & $<1$ \\
\hline & $9 / 22 / 88$ & $<10$ & $<1$ & $<1$ & $<1$ & $<1$ & $<1$ & $<1$ & $<1$ & $<1$ & $<1$ \\
\hline & $8 / 16 / 89$ & $<10$ & $<1$ & $<1$ & $<1$ & $<1$ & $<1$ & $<1$ & $<1$ & $<1$ & $<1$ \\
\hline \multirow[t]{3}{*}{$D L-14 A$} & $8 / 21 / 88$ & $<10$ & $<1$ & $<1$ & $<1$ & $<1$ & $<1$ & $<1$ & $<1$ & $100 \pm 10$ & $<1$ \\
\hline & $9 / 22 / 88$ & $<10$ & $<1$ & $<1$ & $<1$ & $<1$ & $<1$ & $<1$ & $<1$ & $<1$ & $<1$ \\
\hline & $8 / 16 / 89$ & $<10$ & $<1$ & $<1$ & $<1$ & $<1$ & $<1$ & $<1$ & $<1$ & $<1$ & $<1$ \\
\hline \multirow[t]{2}{*}{ MF -5} & $8 / 21 / 88$ & $<10$ & $<1$ & $<1$ & $<1$ & $<1$ & $<1$ & $<1$ & $<1$ & $100 \pm 10$ & $<1$ \\
\hline & $8 / 16 / 89$ & $<10$ & $<1$ & $<1$ & $<1$ & $<1$ & $<1$ & $<1$ & $<1$ & $<1$ & $<1$ \\
\hline \multirow[t]{3}{*}{$M F-6$} & $8 / 21 / 88$ & $<10$ & $<:$ & $<1$ & $<1$ & $<1$ & $<1$ & $<1$ & $<1$ & $100 \pm 10$ & $<1$ \\
\hline & $9 / 22 / 88$ & $<10$ & $<1$ & $<1$ & $<1$ & $<1$ & $<1$ & $<1$ & $<1$ & $<1$ & $<1$ \\
\hline & $8 / 16 / 89$ & $<10$ & $<1$ & $<1$ & $<1$ & $<1$ & $<1$ & $<1$ & $<1$ & $<1$ & $<1$ \\
\hline \multirow[t]{3}{*}{ MF- 8} & $8 / 21 / 88$ & $<10$ & $<1$ & $<1$ & $<1$ & $<1$ & $<1$ & $<1$ & $<1$ & $100 \pm 10$ & $<1$ \\
\hline & $9 / 22 / 88$ & $<10$ & $<1$ & $<1$ & $<1$ & $<1$ & $<1$ & $<1$ & $<1$ & $<1$ & $<1$ \\
\hline & $8 / 16 / 89$ & $<10$ & $<1$ & $<1$ & $<1$ & $<1$ & $<1$ & $<1$ & $<1$ & $<1$ & $<1$ \\
\hline MF- 9 & $9 / 22 / 88$ & $<10$ & $<1$ & $<1$ & $<1$ & $<1$ & $<1$ & -1 & $<1$ & $<1$ & $<1$ \\
\hline \multirow[t]{3}{*}{$T F-2$} & $8 / 21 / 88$ & $<10$ & $<1$ & $<1$ & $<1$ & $<1$ & $<1$ & $<1$ & $<1$ & $100 \pm 10$ & $<1$ \\
\hline & $9 / 22 / 88$ & $<10$ & $<1$ & $<1$ & $<1$ & $<1$ & $<1$ & $<1$ & $<1$ & $<1$ & $<1$ \\
\hline & $8 / 16 / 89$ & $<10$ & $<1$ & $<1$ & $<1$ & $<1$ & $<1$ & $<1$ & $<1$ & $<1$ & $<1$ \\
\hline \multirow[t]{2}{*}{ TF -4} & $8 / 21 / 88$ & $<10$ & $<1$ & $<1$ & $10 \pm 1$ & $<1$ & $<1$ & $<1$ & $<1$ & $100 \pm 10$ & $<1$ \\
\hline & $9 / 22 / 88$ & $<10$ & $<1$ & $<1$ & $<1$ & $<1$ & $<1$ & $<1$ & $<1$ & $<1$ & $<1$ \\
\hline \multirow[t]{3}{*}{$T F=7$} & $8 / 21 / 88$ & $<10$ & $<1$ & $<1$ & $5 \pm 1$ & $<1$ & $<1$ & $<1$ & $<1$ & $100 \pm 10$ & $<1$ \\
\hline & $9 / 22 / 88$ & $87 \pm 17$ & $<1$ & $<1$ & $<1$ & $<1$ & $<1$ & $<1$ & $<1$ & $<1$ & $<1$ \\
\hline & $8 / 16 / 89$ & $<10$ & $<1$ & $<1$ & $<1$ & $<1$ & $<1$ & $<1$ & $<1$ & $<1$ & $<1$ \\
\hline \multirow[t]{3}{*}{$T F-11$} & $8 / 21 / 88$ & $<10$ & $<1$ & $<1$ & $<1$ & $<1$ & $<1$ & $<1$ & $<1$ & $100 \pm 10$ & $<1$ \\
\hline & $9 / 22 / 88$ & $<10$ & $<1$ & $<1$ & $<1$ & $<1$ & $<1$ & $<1$ & $<1$ & $<1$ & $<1$ \\
\hline & $8 / 16 / 89$ & $<10$ & $<1$ & $<1$ & $<1$ & $<1$ & $<1$ & $<1$ & $<1$ & $<1$ & $<1$ \\
\hline \multirow[t]{3}{*}{$T F-14$} & $8 / 21 / 88$ & $<10$ & $<1$ & $<1$ & $<1$ & $<1$ & $<1$ & $<1$ & $<1$ & $100 \pm 10$ & $<1$ \\
\hline & $9 / 22 / 88$ & $<10$ & $<1$ & $<1$ & $<1$ & $<1$ & $<1$ & $<1$ & $<1$ & $<1$ & $<1$ \\
\hline & $8 / 16 / 89$ & $<10$ & $<1$ & $<1$ & $<1$ & $<1$ & $<1$ & $<1$ & $<1$ & $<i$ & $<1$ \\
\hline
\end{tabular}




\begin{tabular}{|c|c|c|c|c|c|c|c|c|c|c|c|c|}
\hline $\begin{array}{c}\text { Site } \\
\text { No. }\end{array}$ & $\begin{array}{c}\text { Collection } \\
\text { Date }\end{array}$ & $\begin{array}{c}A g \\
(u g / L)\end{array}$ & $\begin{array}{c}A l \\
(u g / L)\end{array}$ & $\begin{array}{c}\text { As } \\
\text { (ug/i) }\end{array}$ & $\begin{array}{c}A u \\
(u g / L)\end{array}$ & $\begin{array}{c}\text { B } \\
(u g / L)\end{array}$ & $\begin{array}{c}B a \\
(u g / L)\end{array}$ & $\begin{array}{c}\text { Be } \\
(u g / L)\end{array}$ & $\begin{array}{c}\mathrm{Bi} \\
(u g / L)\end{array}$ & $\begin{array}{c}B r \\
(u g / L)\end{array}$ & $\begin{array}{c}\mathrm{Ca} \\
(\mathrm{mg} / \mathrm{L})\end{array}$ & $\begin{array}{l}\text { Cd } \\
(u g / L)\end{array}$ \\
\hline $\begin{array}{l}\text { LAKE - } 1 \\
\text { CAT - } 1 \\
\text { CAT - } 2 \\
\text { CAT - 3 } \\
\text { CAT - } 4 \\
\text { CAT - } 5 \\
\text { GRAPE - } 1 \\
\text { GRAPE - } 2\end{array}$ & $\begin{array}{l}05 / 29 / 90 \\
05 / 29 / 90 \\
05 / 29 / 90 \\
05 / 29 / 90 \\
05 / 29 / 90 \\
05 / 29 / 90 \\
05 / 31 / 90 \\
05 / 31 / 90\end{array}$ & $\begin{aligned} 18 & \pm 2 \\
& <1 \\
& <1 \\
& <1 \\
& <1 \\
& <1 \\
& <1 \\
& <1\end{aligned}$ & $\begin{array}{l}\cdots \\
\ldots \\
\ldots \\
\ldots \\
\ldots \\
\ldots \\
\ldots \\
\ldots\end{array}$ & $\begin{array}{l}<10 \\
<10 \\
<10 \\
<10 \\
<10 \\
<10 \\
<10 \\
<10\end{array}$ & $\begin{array}{l}<1 \\
<1 \\
<1 \\
<1 \\
<1 \\
<1 \\
<1 \\
<1\end{array}$ & $\begin{array}{l}<100 \\
<100 \\
<100 \\
<100 \\
<100 \\
<100 \\
<100 \\
<100\end{array}$ & $\begin{array}{l}<100 \\
<100 \\
<100 \\
<100 \\
<100 \\
<100 \\
<100 \\
<100\end{array}$ & $\begin{array}{l}<10 \\
<10 \\
<10 \\
<10 \\
<10 \\
<10 \\
<10 \\
<10\end{array}$ & $\begin{array}{l}<1 \\
<1 \\
<1 \\
<1 \\
<1 \\
<1 \\
<1 \\
<1\end{array}$ & $\begin{array}{l}<100 \\
<100 \\
<100 \\
<100 \\
<100 \\
<100 \\
<100 \\
<100\end{array}$ & $\begin{array}{l}12 \pm 2 \\
13 \pm 2 \\
12 \pm 2 \\
15 \pm 3 \\
12 \pm 2 \\
11 \pm ? \\
14 \pm 2 \\
16 \pm 3\end{array}$ & $\begin{aligned}< & 0.1 \\
< & 0.1 \\
< & 0.1 \\
< & 0.1 \\
< & 0.1 \\
< & 0.1 \\
< & 0.1 \\
0.2 & \pm 0.1\end{aligned}$ \\
\hline
\end{tabular}

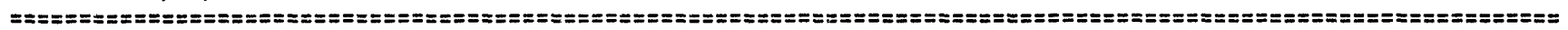

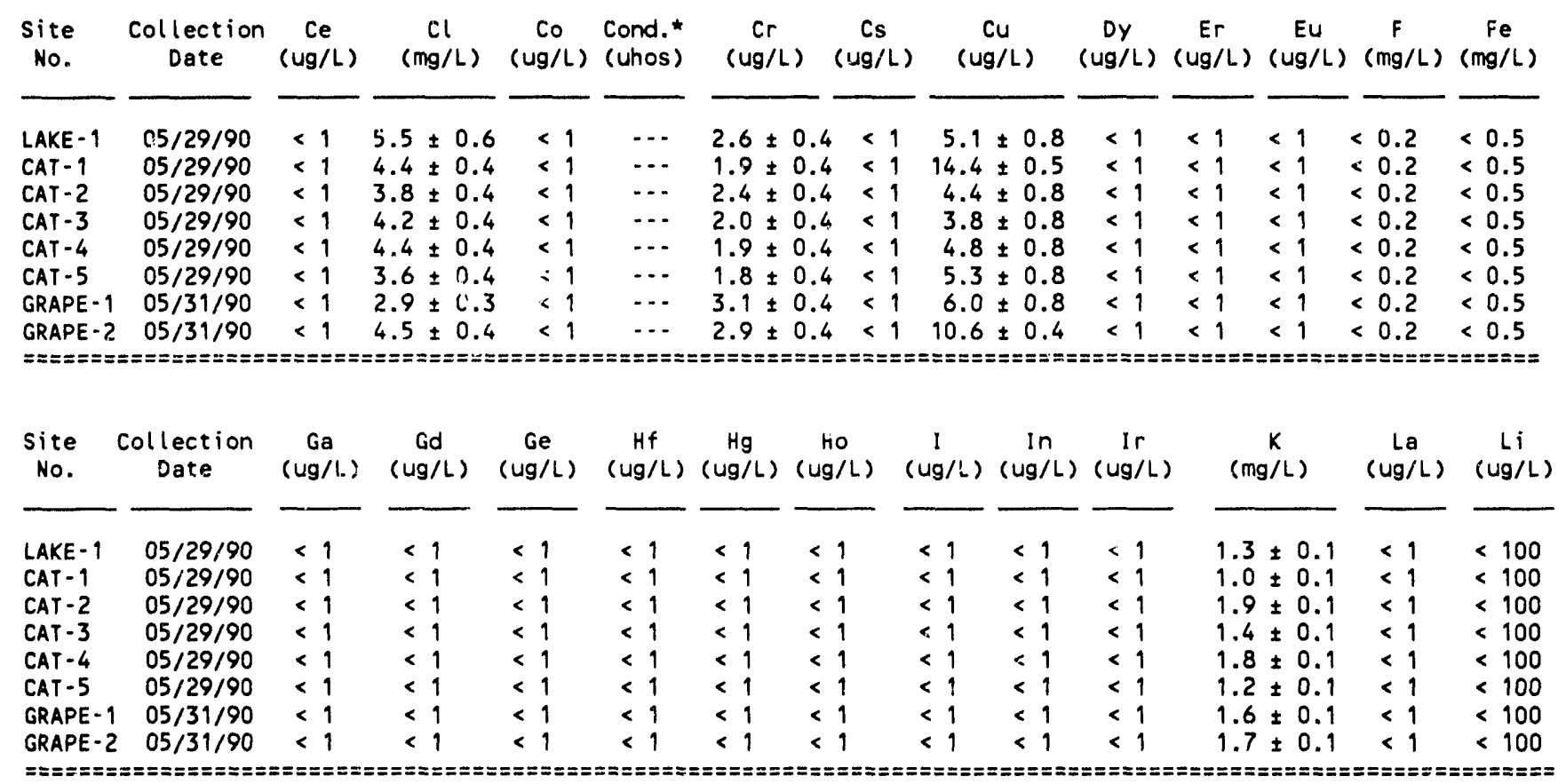

\begin{tabular}{|c|c|c|c|c|c|c|c|c|c|c|c|}
\hline $\begin{array}{l}\text { Site } \\
\text { No. }\end{array}$ & $\begin{array}{c}\text { Collection } \\
\text { Date }\end{array}$ & $\begin{array}{c}\mathrm{Lu} \\
(u g / L)\end{array}$ & $\begin{array}{c}M g \\
(m g / L)\end{array}$ & $\begin{array}{c}\text { Mn } \\
(u g / L)\end{array}$ & $\begin{array}{c}\text { Mo } \\
(u g / L)\end{array}$ & $\begin{array}{c}\mathrm{Na} \\
(\mathrm{mg} / \mathrm{L})\end{array}$ & $\begin{array}{c}\mathrm{Nb} \\
\text { (ug/L) }\end{array}$ & $\begin{array}{c}\text { Nd } \\
\text { (ug/L) }\end{array}$ & $\begin{array}{c}N i \\
(u g / L)\end{array}$ & $\begin{array}{l}\mathrm{NO}_{3}-\mathrm{N} \\
(\mathrm{mg} / \mathrm{L})\end{array}$ & $\begin{array}{c}\text { Os } \\
(u g / L)\end{array}$ \\
\hline LAKE - 1 & $05 / 29 / 90$ & $<1$ & $1.8 \pm 0.1$ & $25 \pm 2$ & $<1$ & $1.4 \pm 0.1$ & $<1$ & $<1$ & $<1$ & $\ldots$ & $<1$ \\
\hline$C A T-1$ & $05 / 29 / 90$ & $<1$ & $1.9 \pm 0.1$ & $65 \pm 1$ & $<1$ & $1.0 \pm 0.1$ & $<1$ & $<1$ & $<1$ & $\ldots$ & $<1$ \\
\hline CAT - 2 & $05 / 29 / 90$ & $<1$ & $1.3 \pm 0.1$ & $3 \pm 1$ & $<1$ & $1.0 \pm 0.1$ & $<1$ & $<1$ & $<1$ & $\ldots$ & $<1$ \\
\hline CAT. 3 & $05 / 29 / 90$ & $<1$ & $1.7 \pm 0.1$ & $10 \pm 1$ & $<1$ & $1.2 \pm 0.1$ & $<1$ & $<1$ & $<1$ & $\cdots$ & $<1$ \\
\hline CAT -4 & $05 / 29 / 90$ & $<1$ & $3.0 \pm 0.4$ & $3 \pm 1$ & $<1$ & $1.3 \pm 0.1$ & $<1$ & $<1$ & $<1$ & $\ldots$ & $<1$ \\
\hline CAT -5 & $05 / 29 / 90$ & $<1$ & $1.9 \pm 0.1$ & $9 \pm 1$ & $<1$ & $1.3 \pm 0.1$ & $<1$ & $<1$ & $<1$ & $\cdots$ & $<1$ \\
\hline GRAPE - 1 & $05 / 31 / 90$ & $<1$ & $2.1 \pm 0.2$ & $3 \pm 1$ & $<1$ & $1.6 \pm 0.2$ & $<1$ & $<1$ & $<1$ & $\cdots$ & $<1$ \\
\hline GRAPE-2 & $05 / 31 / 90$ & $<1$ & $2.3 \pm 0.2$ & $16 \pm 2$ & $<1$ & $1.7 \pm 0.2$ & $<1$ & $<1$ & $<1$ & $\cdots$ & $<1$ \\
\hline
\end{tabular}

* Cond. = Conductivity 


\begin{tabular}{|c|c|c|c|c|c|c|c|c|c|c|c|c|}
\hline $\begin{array}{c}\text { Site } \\
\text { No. }\end{array}$ & $\begin{array}{c}\text { Collection } \\
\text { Date }\end{array}$ & $\begin{array}{c}\mathrm{Pb} \\
(u g / L)\end{array}$ & $\begin{array}{c}\text { Pd } \\
(u g / L)\end{array}$ & $\begin{array}{l}\mathrm{PO}_{4}-\mathrm{P} \\
(\mathrm{mg} / \mathrm{L})\end{array}$ & $\begin{array}{c}\operatorname{Pr} \\
(u g / L)\end{array}$ & $\begin{array}{c}P t \\
(u g / L)\end{array}$ & $\begin{array}{c}\mathrm{Rb} \\
(u g / L)\end{array}$ & $\begin{array}{c}\operatorname{Re} \\
(u g / L)\end{array}$ & $\begin{array}{c}\mathrm{Rh} \\
(u g / L)\end{array}$ & $\begin{array}{c}R u \\
(u g / L)\end{array}$ & $\begin{array}{c}S b \\
(u g / L)\end{array}$ & $\begin{array}{c}\text { Sc } \\
(u g / L)\end{array}$ \\
\hline LAKE- 1 & $05 / 29 / 90$ & $<2$ & $<1$ & -- & $<1$ & $<1$ & $<1$ & $<1$ & $<1$ & $<1$ & $<1$ & $<1$ \\
\hline CAT-1 & $05 / 29 / 90$ & $<2$ & $<1$ & -- & $<1$ & $<1$ & $<1$ & $<1$ & $<1$ & $<1$ & $<1$ & $<1$ \\
\hline CAT-2 & $05 / 29 / 90$ & $<2$ & $<1$ & $-\cdots$ & $<1$ & $<1$ & $<1$ & $<1$ & $<1$ & $<1$ & $<1$ & $<1$ \\
\hline CAT-3 & $05 / 29 / 90$ & $<2$ & $<1$ & --- & $<1$ & $<1$ & $<1$ & $<1$ & $<1$ & $<1$ & $<1$ & $<1$ \\
\hline CAT -4 & $05 / 29 / 90$ & $<2$ & $<1$ & $\cdots$ & $<1$ & $<1$ & $<1$ & $<1$ & $<1$ & $<1$ & $<1$ & $<1$ \\
\hline CAT -5 & $05 / 29 / 90$ & $<2$ & $<1$ & $\cdots$ & $<1$ & $<1$ & $<1$ & $<1$ & $<1$ & $<1$ & $<1$ & $<1$ \\
\hline GRAPE - 1 & $05 / 31 / 90$ & $<2$ & $<1$ & -- & $<1$ & $<1$ & $<1$ & $<1$ & $<1$ & $<1$ & $<1$ & $<1$ \\
\hline GRAPE -2 & $05 / 31 / 90$ & $<2$ & $<1$ & --- & $<1$ & $<1$ & $<1$ & $<1$ & $<1$ & $<1$ & $<1$ & $<1$ \\
\hline
\end{tabular}

\begin{tabular}{|c|c|c|c|c|c|c|c|c|c|c|c|}
\hline $\begin{array}{l}\text { Site } \\
\text { No. }\end{array}$ & $\begin{array}{c}\text { Collection } \\
\text { Date }\end{array}$ & $\begin{array}{c}\text { Se } \\
(u g / L)\end{array}$ & $\begin{array}{c}\mathrm{Sm} \\
(u g / L)\end{array}$ & $\begin{array}{c}\mathrm{Sn} \\
(\mathrm{ug} / \mathrm{L})\end{array}$ & $\begin{array}{l}\mathrm{SO}_{4} \\
(\mathrm{mg} / \mathrm{L})\end{array}$ & $\begin{array}{c}S r \\
(u g / L)\end{array}$ & $\begin{array}{c}\text { Ta } \\
(u g / L)\end{array}$ & $\begin{array}{l}\text { T Alk.* } \\
(\mathrm{mg} / \mathrm{L})\end{array}$ & $\begin{array}{c}\mathrm{Tb} \\
(\mathrm{ug} / \mathrm{L})\end{array}$ & $\begin{array}{c}\text { Te } \\
(u g / L)\end{array}$ & $\begin{array}{c}\text { Th } \\
(\mathrm{ug} / \mathrm{L})\end{array}$ \\
\hline LAKE - 1 & $05 / 29 / 90$ & $<10$ & $<1$ & $<1$ & $22 \pm 3$ & $100 \pm 15$ & $<1$ & -- & $<1$ & $<1$ & $<1$ \\
\hline CAT-1 & $05 / 29 / 90$ & $<10$ & $<1$ & $<1$ & $21 \pm 2$ & $88 \pm 10$ & $<1$ & --- & $<1$ & $<1$ & $<1$ \\
\hline CAT-2 & $05 / 29 / 90$ & $<10$ & $<1$ & $<1$ & $25 \pm 2$ & $42 \pm 4$ & $<1$ & --- & $<1$ & $<1$ & $<1$ \\
\hline CAT -3 & $05 / 29 / 90$ & $<10$ & $<1$ & $<1$ & $31 \pm 3$ & $39 \pm 1$ & $<1$ & --- & $<1$ & $<1$ & $<1$ \\
\hline CAT- 4 & $05 / 29 / 90$ & $<10$ & $<1$ & $<1$ & $24 \pm 2$ & $49 \pm 3$ & $<1$ & $\cdots$ & $<1$ & $<1$ & $<1$ \\
\hline$C A T-5$ & $05 / 29 / 90$ & $<10$ & $<1$ & $<1$ & $24 \pm 3$ & $66 \pm 1$ & $<1$ & $\cdots$ & $<1$ & $<1$ & $<1$ \\
\hline GRAPE - 1 & $05 / 31 / 90$ & $<10$ & $<1$ & $<1$ & $25 \pm 2$ & $100 \pm 15$ & $<1$ & --- & $<1$ & $<1$ & $<1$ \\
\hline GRAPE - 2 & $05 / 31 / 90$ & $<10$ & $<1$ & $<1$ & $27 \pm 3$ & $88 \pm 10$ & $<1$ & -- & $<1$ & $<1$ & $<1$ \\
\hline
\end{tabular}

\begin{tabular}{|c|c|c|c|c|c|c|c|c|c|c|c|}
\hline $\begin{array}{l}\text { Site } \\
\text { No. }\end{array}$ & $\begin{array}{c}\text { Collection } \\
\text { Date }\end{array}$ & $\begin{array}{c}\mathrm{Ti} \\
(u g / L)\end{array}$ & $\begin{array}{c}T L \\
(u g / L)\end{array}$ & $\begin{array}{c}\mathrm{Tm} \\
(\mathrm{ug} / \mathrm{L})\end{array}$ & $\begin{array}{c}U \\
(u g / L)\end{array}$ & $\begin{array}{c}V \\
(u g / L)\end{array}$ & $\begin{array}{c}W \\
(u g / L)\end{array}$ & $\begin{array}{c}Y \\
(u g / L)\end{array}$ & $\begin{array}{l}Y b \\
(u g / L)\end{array}$ & $\begin{array}{c}\mathrm{Zn} \\
(\mathrm{ug} / \mathrm{L})\end{array}$ & $\begin{array}{c}\mathrm{Zr} \\
(\mathrm{ug} / \mathrm{L})\end{array}$ \\
\hline LAKE- 1 & $05 / 29 / 90$ & $<10$ & $<1$ & $<1$ & $<1$ & $<1$ & $<1$ & $<1$ & $<1$ & $47 \pm 7$ & $<1$ \\
\hline CAT-1 & $05 / 29 / 90$ & $<10$ & $<1$ & $<1$ & $<1$ & $<1$ & $<1$ & $<1$ & $<1$ & $23 \pm 13$ & $<1$ \\
\hline CAT-2 & $05 / 29 / 90$ & $<10$ & $<1$ & $<1$ & $<1$ & $<1$ & $<1$ & $<1$ & $<1$ & $5 \pm 7$ & $<1$ \\
\hline CAT-3 & $05 / 29 / 90$ & $<10$ & $<1$ & $<1$ & $<1$ & $<1$ & $<1$ & $<1$ & $<1$ & $9 \pm 6$ & $<1$ \\
\hline CAT-4 & $05 / 29 / 90$ & $<10$ & $<1$ & $<1$ & $<1$ & $<1$ & $<1$ & $<1$ & $<1$ & $12 \pm 7$ & $<1$ \\
\hline$C A T-5$ & $05 / 29 / 90$ & $<10$ & $<1$ & $<1$ & $<1$ & $<1$ & $<1$ & $<1$ & $<1$ & $23 \pm 18$ & $<1$ \\
\hline GRAPE-1 & $05 / 31 / 90$ & $<10$ & $<1$ & $<1$ & $<1$ & $<1$ & $<1$ & $<1$ & $<1$ & $46 \pm 11$ & $<1$ \\
\hline GRAPE-2 & $05 / 31 / 90$ & $<10$ & $<1$ & $<1$ & $<1$ & $<1$ & $<1$ & $<1$ & $<1$ & $15 \pm 6$ & $<1$ \\
\hline
\end{tabular}

* T Alk. = Total Alkalinity 


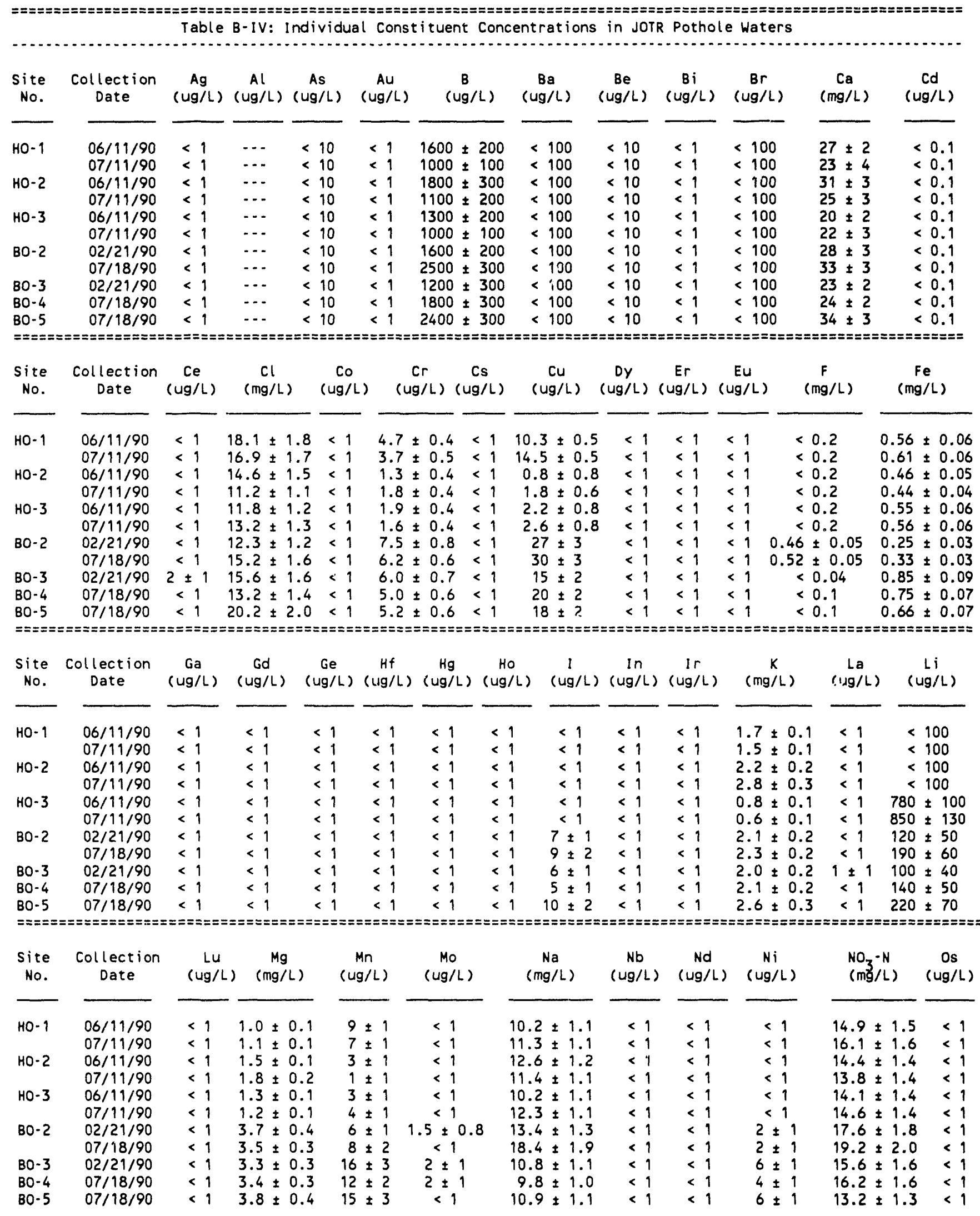

*ond. = Conductivity 
Table B-IV: Individual Constituent Concentrations in JOTR Pothole Waters (cont.)

\begin{tabular}{|c|c|c|c|c|c|c|c|c|c|c|c|c|}
\hline $\begin{array}{l}\text { Site } \\
\text { No. }\end{array}$ & $\begin{array}{c}\text { Collection } \\
\text { Date }\end{array}$ & $\begin{array}{c}\mathrm{Pb} \\
\text { (ug/L) }\end{array}$ & $\begin{array}{c}P d \\
\text { (ug/L) }\end{array}$ & $\begin{array}{l}\mathrm{PO}-\mathrm{P} \\
(\mathrm{mg} / \mathrm{L})\end{array}$ & $\begin{array}{c}\mathrm{Pr} \\
\text { (ug/L) }\end{array}$ & $\begin{array}{c}\text { Pt } \\
(u g / L)\end{array}$ & $\begin{array}{c}\mathrm{Rb} \\
(\mathrm{ug} / \mathrm{L})\end{array}$ & $\begin{array}{c}\operatorname{Re} \\
(\mathrm{ug} / \mathrm{L})\end{array}$ & $\begin{array}{c}\mathrm{Rh} \\
(\mathrm{ug} / \mathrm{L})\end{array}$ & $\begin{array}{c}\mathrm{Ru} \\
\text { (ug/L) }\end{array}$ & $\begin{array}{c}S b \\
(u g / L)\end{array}$ & $\begin{array}{c}\text { Si: } \\
(u g /-)\end{array}$ \\
\hline \multirow[t]{2}{*}{ HO-1 } & $06 / 11 / 90$ & $<2$ & $<1$ & -- & $<1$ & $<1$ & $<1$ & $<1$ & $<1$ & $<1$ & $<1$ & $<1$ \\
\hline & $07 / 11 / 90$ & $<2$ & $<1$ & -- & $<1$ & $<1$ & $<1$ & $<1$ & $<1$ & $<1$ & $<1$ & $<1$ \\
\hline \multirow[t]{2}{*}{ HO-2 } & $06 / 11 / 90$ & $<2$ & $<1$ & -- & $<1$ & $<1$ & $<1$ & $<1$ & $<1$ & $<1$ & $<1$ & $<1$ \\
\hline & $07 / 11 / 90$ & $<2$ & $<1$ & -- & $<1$ & $<:$ & $<1$ & $<1$ & $<1$ & $<1$ & $<1$ & $<1$ \\
\hline \multirow[t]{2}{*}{$\mathrm{HO}-3$} & $06 / 11 / 90$ & $<2$ & $<1$ & $\cdots$ & $<1$ & $<1$ & $<1$ & $<1$ & $<1$ & $<1$ & $<1$ & $<1$ \\
\hline & $07 / 11 / 90$ & $<2$ & $<1$ & -- & $<1$ & $<1$ & $<1$ & $<1$ & $<1$ & $<1$ & $<1$ & $<1$ \\
\hline \multirow[t]{2}{*}{ BO-2 } & $02 / 21 / 90$ & $26 \pm 4$ & $<1$ & $<0.2$ & $<1$ & $<1$ & $2 \pm 1$ & $<1$ & $<1$ & $<1$ & $<1$ & $<1$ \\
\hline & $07 / 18 / 90$ & $35 \pm 5$ & $<1$ & $<0.2$ & $<1$ & $<1$ & $4 \pm 1$ & $<1$ & $<1$ & $<1$ & $<1$ & $<1$ \\
\hline BO- 3 & $02 / 21 / 90$ & $19 \pm 3$ & $<1$ & $0.6 \pm 0.2$ & $<1$ & $<1$ & $3 \pm 1$ & $<1$ & $<1$ & $<1$ & $<1$ & $<1$ \\
\hline BO-4 & $07 / 18 / 90$ & $18 \pm 3$ & $<1$ & $0.5 \pm 0.2$ & $<1$ & $<1$ & $2 \pm 1$ & $<1$ & $<1$ & $<1$ & $<1$ & $<1$ \\
\hline BO-5 & $07 / 18 / 90$ & $25 \pm 3$ & $<1$ & $0.6 \pm 0.2$ & $<1$ & $<1$ & $3 \pm 1$ & $<1$ & $<1$ & $<1$ & $<1$ & $<1$ \\
\hline
\end{tabular}

\begin{tabular}{|c|c|c|c|c|c|c|c|c|c|c|c|}
\hline $\begin{array}{l}\text { Site } \\
\text { No. }\end{array}$ & $\begin{array}{c}\text { Crllection } \\
\text { Date }\end{array}$ & $\begin{array}{c}\mathrm{Se} \\
(\mathrm{ug} / \mathrm{L})\end{array}$ & $\begin{array}{c}\mathrm{Sm} \\
(\mathrm{ug} / \mathrm{L})\end{array}$ & $\begin{array}{c}\mathrm{Sn} \\
(\mathrm{ug} / \mathrm{L})\end{array}$ & $\begin{array}{l}\mathrm{SO}_{4}^{4} \\
\left(\mathrm{mg}^{4} / \mathrm{L}\right)\end{array}$ & $\begin{array}{c}\mathrm{Sr} \\
(\mathrm{ug} / \mathrm{L})\end{array}$ & $\begin{array}{c}\mathrm{Ta} \\
\text { (ug/L) }\end{array}$ & $\begin{array}{l}\text { T Alk.* } \\
(\mathrm{mg} / \mathrm{L})\end{array}$ & $\begin{array}{c}\mathrm{Tb} \\
(\mathrm{ug} / \mathrm{L})\end{array}$ & $\begin{array}{c}\text { Te } \\
(\mathrm{ug} / \mathrm{L})\end{array}$ & $\begin{array}{c}\text { Th } \\
(u g / L)\end{array}$ \\
\hline \multirow[t]{2}{*}{ HO-1 } & $06 / 11 / 90$ & $<10$ & $<1$ & $<1$ & $25 \pm 2$ & $110 \pm 10$ & $<1$ & -- & $<1$ & $<1$ & $<1$ \\
\hline & $07 / 11 / 90$ & $<10$ & $<1$ & $<1$ & $33 \pm 3$ & $150 \pm 20$ & $<1$ & --- & $<1$ & $<1$ & $<1$ \\
\hline \multirow[t]{2}{*}{ HO-2 } & $06 / 11 / 90$ & $<10$ & $<1$ & $<1$ & $38 \pm 4$ & $44 \pm 6$ & $<1$ & --- & $<1$ & $<1$ & $<1$ \\
\hline & $07 / 11 / 90$ & $<10$ & $<1$ & $<1$ & $42 \pm 4$ & $55 \pm 8$ & $<1$ & --- & $<1$ & $<1$ & $<1$ \\
\hline \multirow[t]{2}{*}{ HO-3 } & $06 / 11 / 90$ & $<10$ & $<1$ & $<1$ & $32 \pm 3$ & $100 \pm 10$ & $<1$ & -- & $<1$ & $<1$ & $<1$ \\
\hline & $07 / 11 / 90$ & $<10$ & $<1$ & $<1$ & $40 \pm 4$ & $90 \pm 10$ & $<1$ & --- & $<1$ & $<1$ & $<1$ \\
\hline \multirow[t]{2}{*}{$\mathrm{BO}-2$} & $02 / 21 / 90$ & $3 \pm 1$ & $<1$ & $6 \pm 1$ & $36 \pm 4$ & $180 \pm 20$ & $<1$ & --- & $<1$ & $<1$ & $<1$ \\
\hline & $07 / 18 / 90$ & $8 \pm 2$ & $<1$ & $5 \pm 1$ & $40 \pm 4$ & $280 \pm 30$ & $<1$ & --- & $<1$ & $<1$ & $<1$ \\
\hline BO- 3 & $02 / 21 / 90$ & $4 \pm 1$ & $<1$ & $5 \pm 1$ & $28 \pm 3$ & $130 \pm 10$ & $<1$ & -- & $<1$ & $<1$ & $<1$ \\
\hline BO-4 & $07 / 18 / 90$ & $4 \pm 1$ & $<1$ & $5 \pm 1$ & $18 \pm 2$ & $150 \pm 20$ & $<1$ & -- & $<1$ & $<1$ & $<1$ \\
\hline BO- 5 & $07 / 18 / 90$ & $4 \pm 1$ & $<1$ & $3 \pm 1$ & $22 \pm 3$ & $140 \pm 10$ & $<1$ & -- & $<1$ & $<1$ & $<1$ \\
\hline $\begin{array}{l}\text { Site } \\
\text { No. }\end{array}$ & $\begin{array}{c}\text { Collection } \\
\text { Date }\end{array}$ & $\begin{array}{c}T i \\
(u g / L)\end{array}$ & $\begin{array}{c}\mathrm{Tl} \\
(\mathrm{ug} / \mathrm{L})\end{array}$ & $\begin{array}{c}\mathrm{Tm} \\
(\mathrm{ug} / \mathrm{L})\end{array}$ & $\begin{array}{c}U \\
(u g / L)\end{array}$ & $\begin{array}{c}V \\
(u g / L)\end{array}$ & $\begin{array}{c}W \\
(u g / L)\end{array}$ & $\begin{array}{c}Y \\
(u g / L)\end{array}$ & $\begin{array}{c}Y b \\
(u g / L)\end{array}$ & $\begin{array}{c}\mathrm{Zn} \\
(\mathrm{ug} / \mathrm{L})\end{array}$ & $\begin{array}{c}\mathrm{Zr} \\
(\mathrm{ug} / \mathrm{L})\end{array}$ \\
\hline \multirow[t]{2}{*}{ HO-1 } & $06 / 11 / 90$ & $<10$ & $<1$ & $<1$ & $<1$ & $<1$ & $<1$ & $<1$ & $<1$ & $13 \pm 8$ & $<1$ \\
\hline & $07 / 11 / 90$ & $<10$ & $<1$ & $<1$ & $<1$ & $<1$ & $<1$ & $<1$ & $<1$ & $<7$ & $<1$ \\
\hline \multirow[t]{2}{*}{ HO-2 } & $06 / 11 / 90$ & $<10$ & $<1$ & $<1$ & $<1$ & $<1$ & $<1$ & $<1$ & $<1$ & $<5$ & $<1$ \\
\hline & $07 / 11 / 90$ & $<10$ & $<1$ & $<1$ & $<1$ & $<1$ & $<1$ & $<1$ & $<1$ & $<5$ & $<1$ \\
\hline \multirow[t]{2}{*}{$\mathrm{HO}-3$} & $06 / 11 / 90$ & $<10$ & $<1$ & $<1$ & $<1$ & $<1$ & $<1$ & $<1$ & $<1$ & $19 \pm 8$ & $<1$ \\
\hline & $07 / 11 / 90$ & $<10$ & $<1$ & $<1$ & $<1$ & $<1$ & $<1$ & $<1$ & $<1$ & $25 \pm 9$ & $<1$ \\
\hline \multirow[t]{2}{*}{ BO-2 } & $02 / 21 / 90$ & $110 \pm 20$ & $<1$ & $<1$ & $<1$ & $1.7 \pm 0.5$ & $2 \pm 1$ & $<1$ & $<1$ & $12 \pm 4$ & $1 \pm 1$ \\
\hline & $07 / 18 / 90$ & $140 \pm 20$ & $<1$ & $<1$ & $<1$ & $1.6 \pm 0.5$ & $1 \pm 1$ & $<1$ & $<1$ & $14 \pm 4$ & $<1$ \\
\hline $\mathrm{BO}-3$ & $02 / 21 / 90$ & $120 \pm 20$ & $<1$ & $<1$ & $<1$ & $3.0 \pm 0.8$ & $1 \pm 1$ & $<1$ & $<1$ & $17 \pm 5$ & $<1$ \\
\hline BO- 4 & $07 / 18 / 90$ & $100 \pm 10$ & $<1$ & $<1$ & $<1$ & $2.9 \pm 0.8$ & $<1$ & $<1$ & $<1$ & $19 \pm 5$ & $<1$ \\
\hline BO-5 & $07 / 18 / 90$ & $100 \pm 10$ & $<1$ & $<1$ & $<1$ & $2.6 \pm 0.7$ & $<1$ & $<1$ & $<1$ & $14 \pm 3$ & $<1$ \\
\hline
\end{tabular}


Appendix C: Individual Elemental Concentrations and Enrichment Factors in Pothole Sediment Samples 


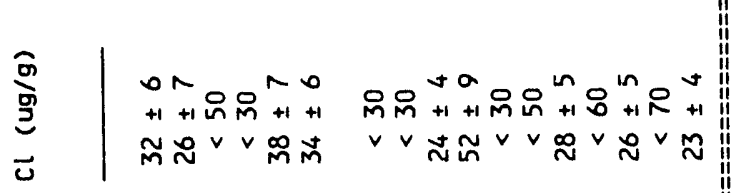

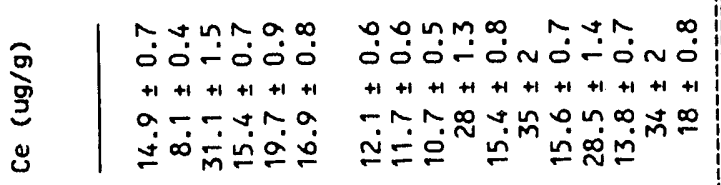

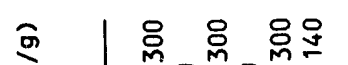

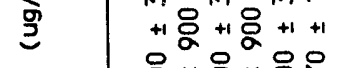
刃

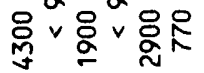

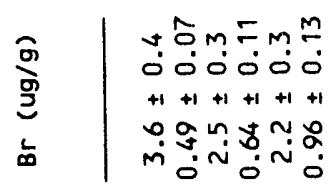

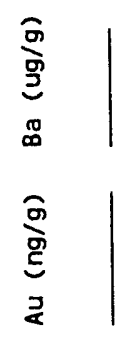

으느ํ붕요요 $+1+1+1+1+1+1$

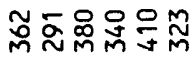

\section{응 옹웡원} 宛各各+1

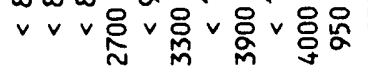

$g=\hat{0}+\sim a \infty n \pm t$

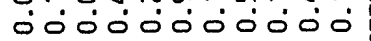
$+1+1+1+1+1+1+1+1+1+1+1$ $M$
$\mathfrak{N}$
0

tนm.

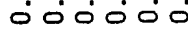
$+1+1+1+1+1+1$ $\div-\infty 0$.

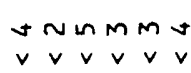

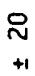

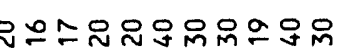
$+1+1+1+1+1+1+1+1+1+1+1$

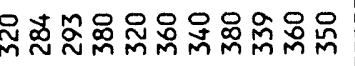

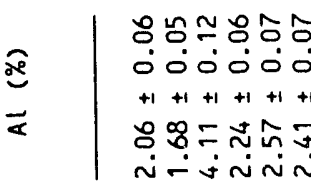

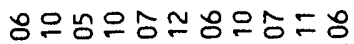
000000000. $+1+1+1+1+1+1+1+1+1+1+1$

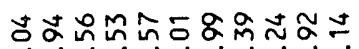
iन- mins-mimi

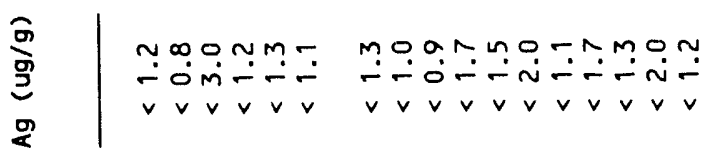

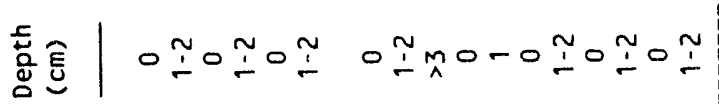

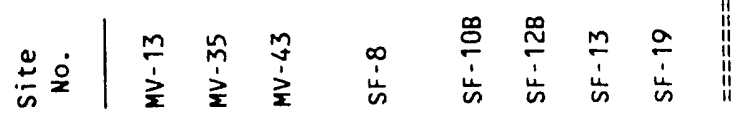

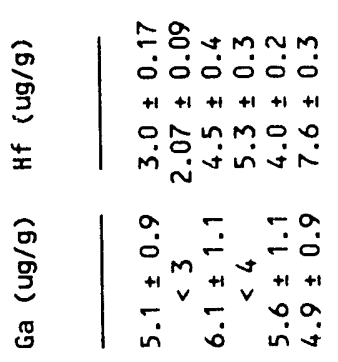

ำบ

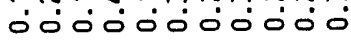
$+1+1+1+1+1+1+1+1+1+1+1$

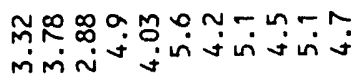

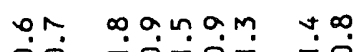
io $\dot{0}-\dot{0}-\dot{0}$ $+1+1 v_{v}^{m}+1+1+1+1+1 v+1+1$

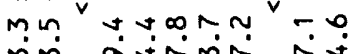

\section{ตัธะธตท} 00000

ริ

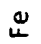

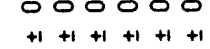
กำำษ 8 年. ठ०⿱宀⿻三丨口 क़

ธัธธณณ லं0ं0்0 $+1+1+1+1+1+1$

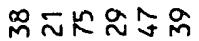
०0ं000

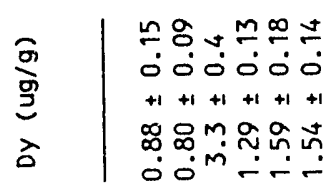

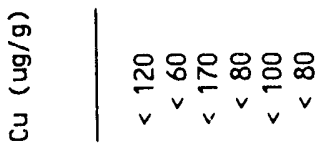

㠃

$=\hat{0} \Delta g \pm \cong$ ऽ००000 $+1+1+1+1+1+1$

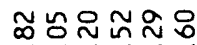
$\because \dot{m}-\dot{\sim}$

命 $\infty+\infty$ 0

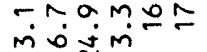

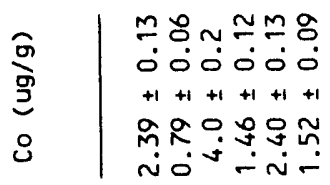

용윤웅ㅇㅇㅛ 000000000 $+1+1+1+1++1+1+1+1+1+1$

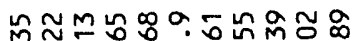

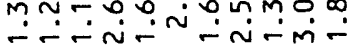

novanavan 00ं $0 \dot{0} \div \dot{0} \div 0 \div-$ $+1+1+1+1+1+1+1+1+1+1+1$ Oommuñ

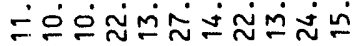

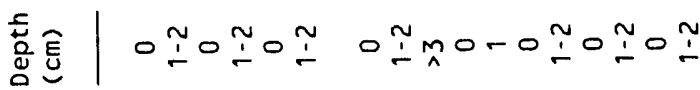

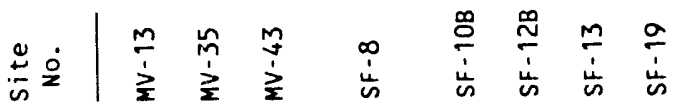




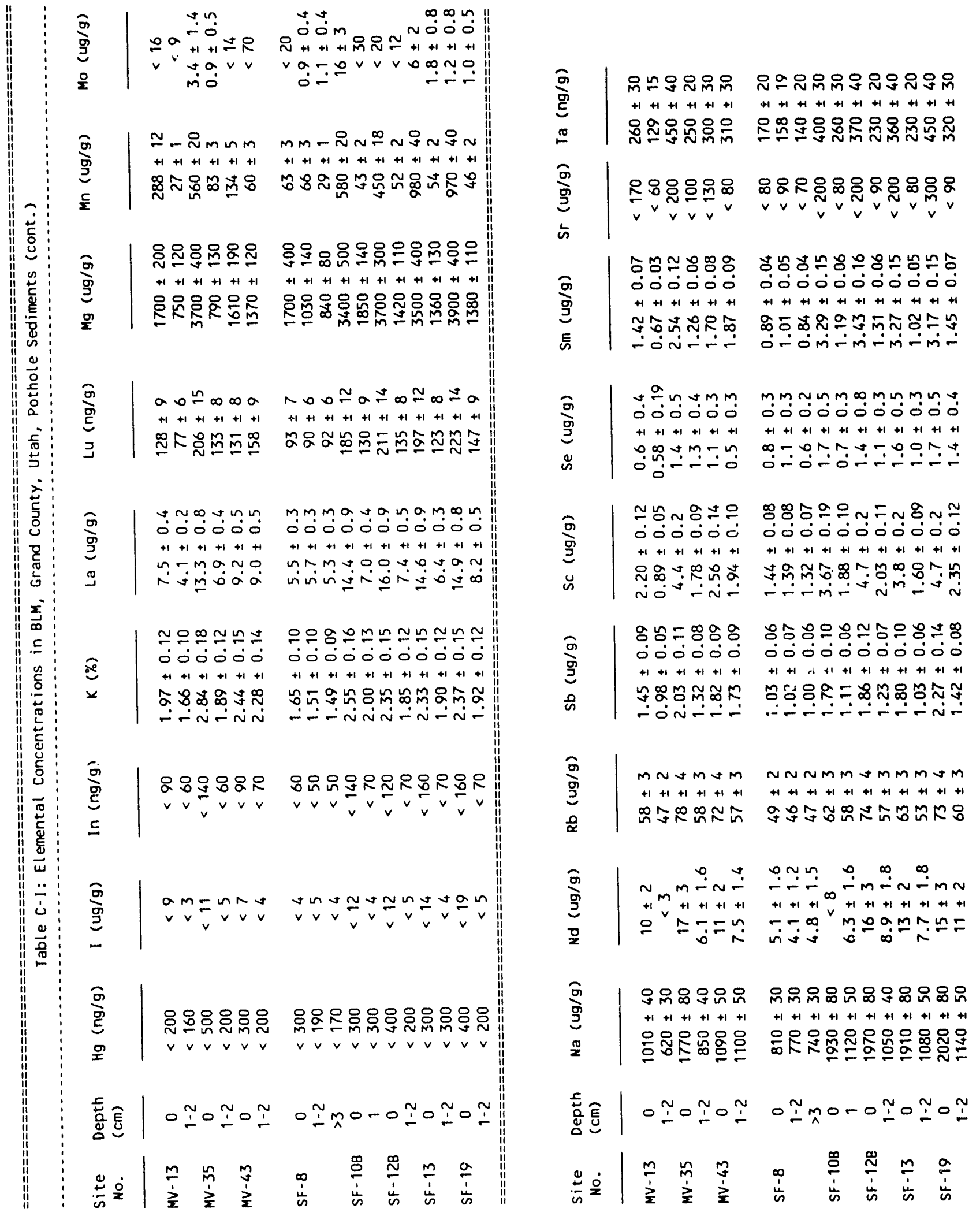




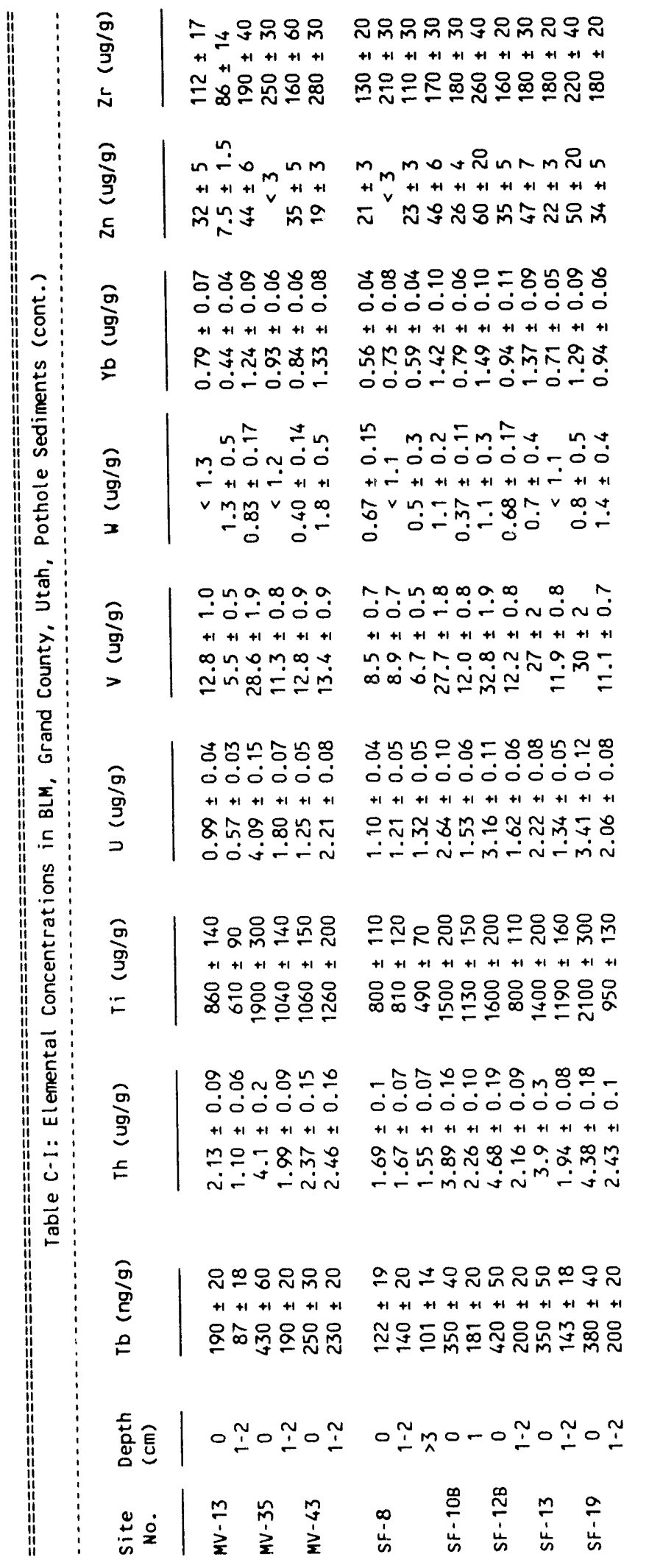




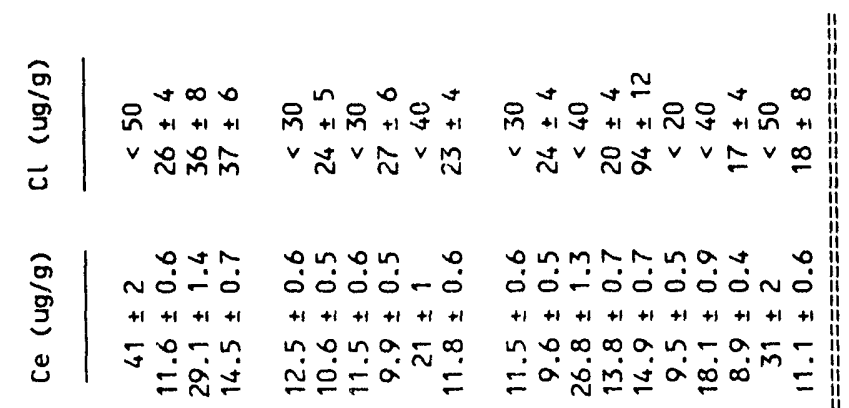

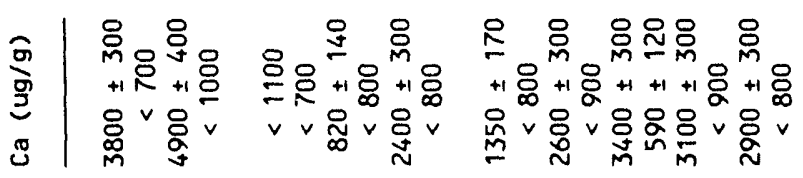

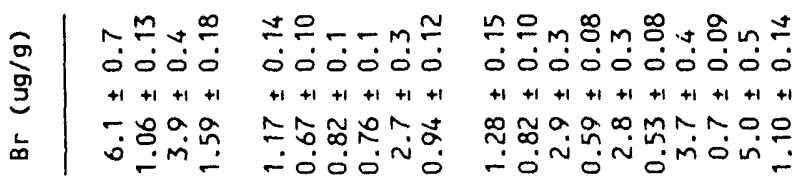

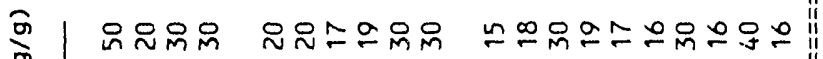
要 $+1+1+1+1+1+1+1+1+1+1+1+1+1+1+1+1+1+1+1$

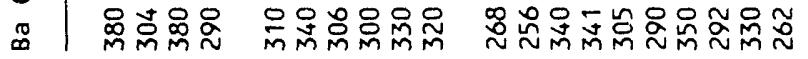

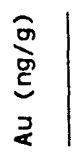

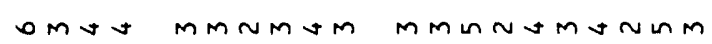
$\vee \vee v \vee v \vee v \vee v \vee v \vee v \vee v \vee v \vee v v$

के

ANOm $m \sim \sim \sim n m$ कं०००

$+1+1+1+1$

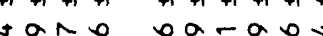

它向向

बह

$\simeq น$ वं००ं व. $+1+1+1+1+1+1+1+1+1+1$

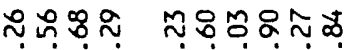
iूmi

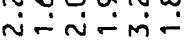

$m \sim n \sim m \bumpeq+m n \sim$ 000000000 $+1+1+1+1+1+1+1+1+1+1$ om $m$ M m N Na-

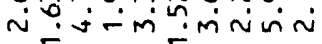

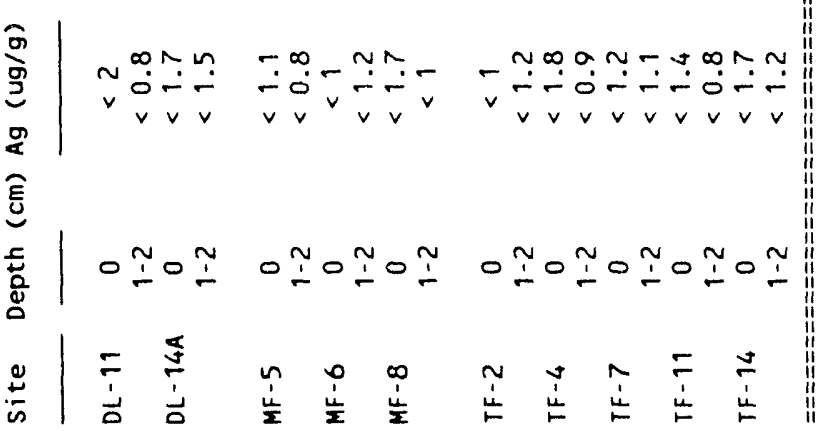

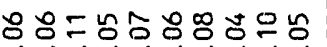

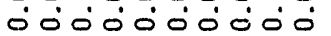
$+1+1+1+1+1+1+1+1+1+1$ ஐ $\because \dot{m}-\dot{\sim}-\dot{\sim}-\dot{m}-$

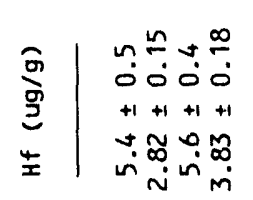

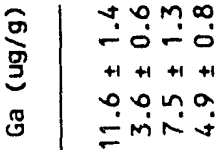

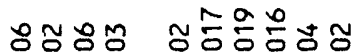

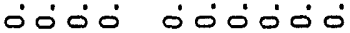

ริ

$\stackrel{4}{4}$

$+1+1+1+1$

సิำํํํ유

के

ชะก

क०००

$+1+1+1+1+1+1+1+1+1+1$

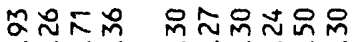

o0ं0 00000

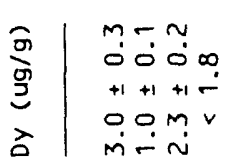

mं-

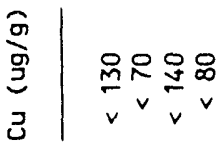

के

N ํํㅇ-

ธ.0.0

$+1+1+1+1$

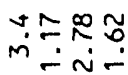

产

की

poot $\infty$ on $-n n$

过

$m \stackrel{\infty}{\circ}==\hat{0} \%$

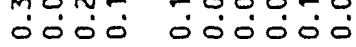

$+1+1+1+1$

in

Eิ

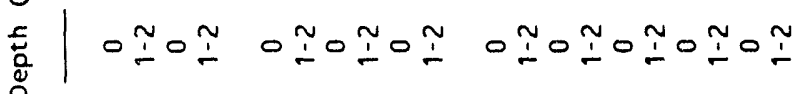

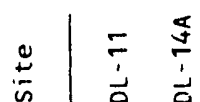

ำ 은 000000 $+1+1+1+1+1+1$

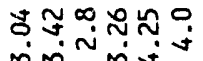

$\infty$ - 0 . . . . o.0.0.

$+1+1+1+1+1+1$

aoninar minisión

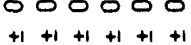

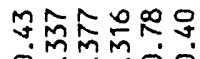

ợmo: ơ:

$\approx \widetilde{\approx} \approx m m$

$00^{\circ} 0^{\circ}$

$m=-0=$

0ं0000

$+1+1+1+1+1+1$

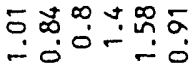

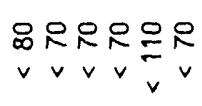

용

000000

$+1+1+1+1+1+1$

m怾品=n

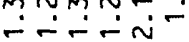

manna

ớ0ல்

$+1+1+1+1+1+1$

$\simeq \dot{0} 0 \dot{0}=$

$+1+1+1+1+1+1$

$m \approx 00 \tilde{m}$

$\sim \sim m m=\longleftarrow ㄴ$ $+1+1+1+1+1+1+1+1+1+1$

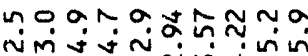

क. $00-0---m m$ $\infty \infty$ in 0 r m o ${ }^{2}{ }^{+} v$

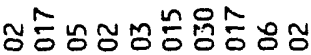
o00000000 $+1+1+1+1+1+1+1+1+1+1$

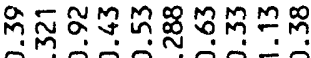

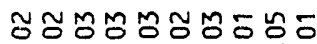
ó000000. $+1+1+1+1+1+1+1+1+1+1$

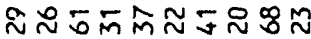
0000000000

$=a \simeq M= \pm N n$ oinosontó0 $+1-+1+1+1-+1+1+1+1$ g.

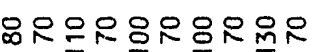
$\checkmark v v_{v} \tau_{v} v v_{v} v v_{v}$

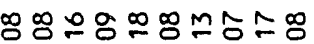
¿00000000 $+1+1+1+1+1+1+1+1+1+1$

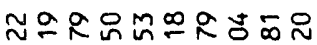
$\because$ -

$\infty, \infty \infty 0$ :0-000-0́n$+1+1+1+1+1+1+1+1+1$

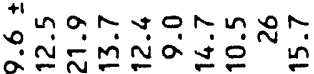

ชิ่ง 000000000 $+1+1+1+1+1+1+1+1+1+1$

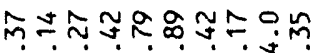

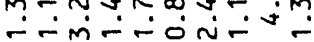

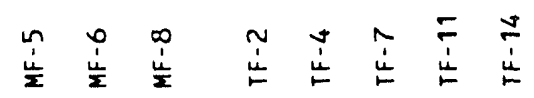
ó0000000 min mivimin 


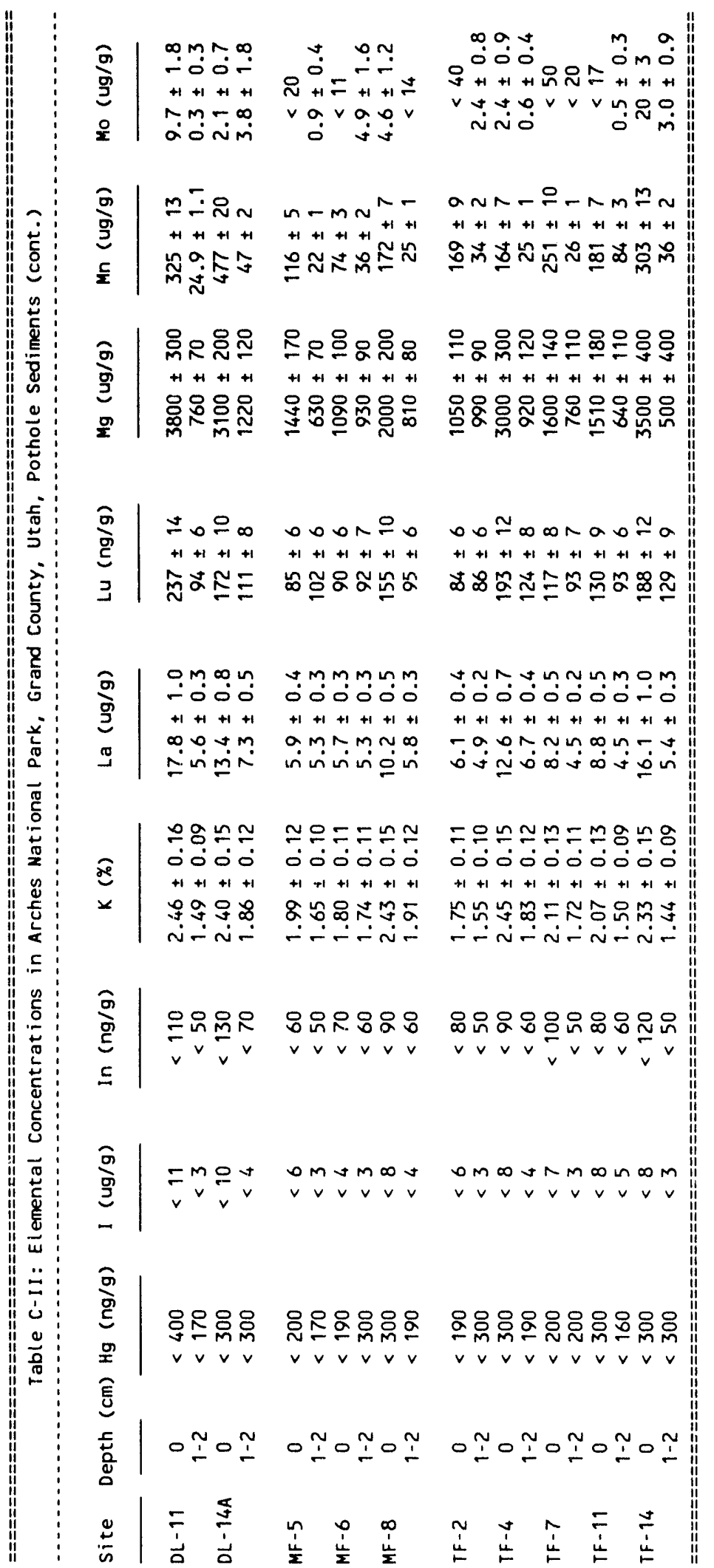

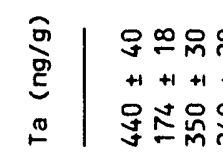

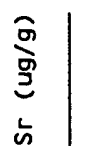

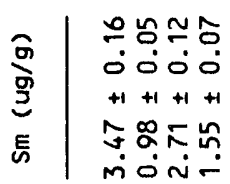

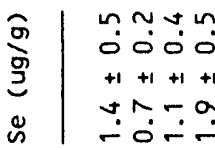

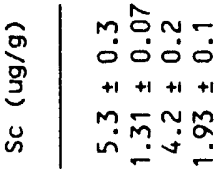

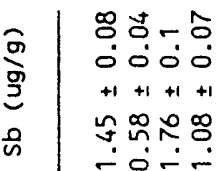

命|

$\checkmark N M M$ $+1+1+1+1$

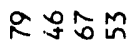

$M M M M \cup M$ $+1+1+1+1+1+1$ ก

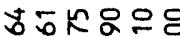

농헝ㄴㅇㅇㄴㅇㅇ영응 वं००ं0 $+1+1+1+1+1+1$

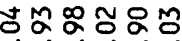

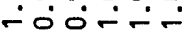

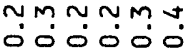
$+1+1+1+1+1+1$ T. ठं 0 ठं $+1+1+1+1+1+1$ $8 \stackrel{0}{0} \stackrel{\infty}{m} \mathfrak{m}$

ํํㅇํํํํㅇㅇำ 0ं0் 000 $+1+1+1+1+1+1$ 유용요

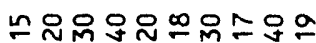

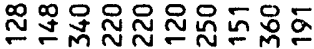

$\operatorname{mos}$

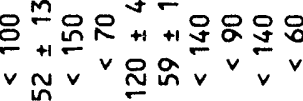

otmunmomo

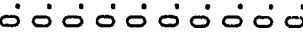
$+1+1+1+1+1+1+1+1+1+1$

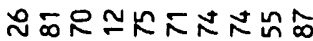

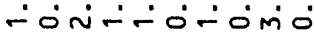

$\sim \sim m \checkmark n M m M M m$

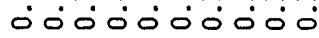
$+1+1+1+1+1+1+1+1+1+1$ in $\infty m 0100-\infty$

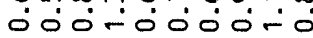

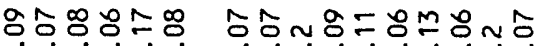
○ं $0 \dot{0} 00^{\circ} 00^{\circ} 0$ $+1+1+1+1+1+1+1+1+1+1$

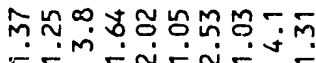
ஸ்ட்-ீ

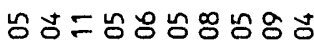
○ं 0 0ं 0000 $+1+1+1+1+1+1+1+1+1+1$

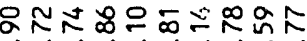

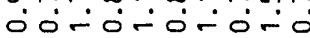

$m \stackrel{0}{-}$

$m-n-$ $\because \infty=0$.

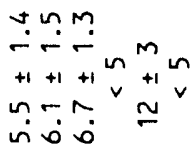

NNOMMMMNMN $+1+1+1+1+1+1+1+1+1+1$

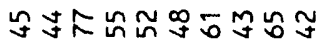

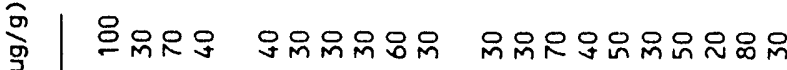
J $+1+1+1+1+1+1+1+1+1+1+1+1+1+1+1+1+1+1+1+1$

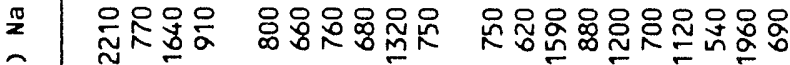
孞 䔄|

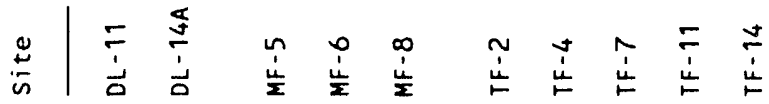




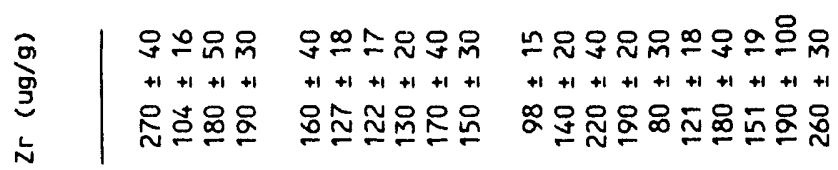

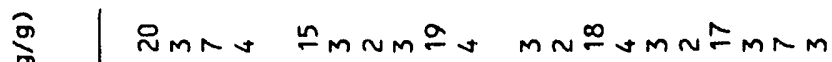

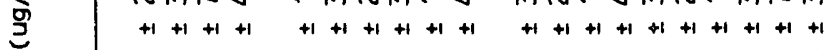

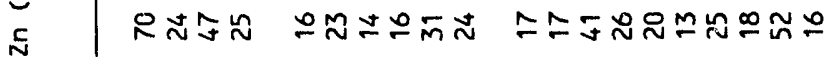

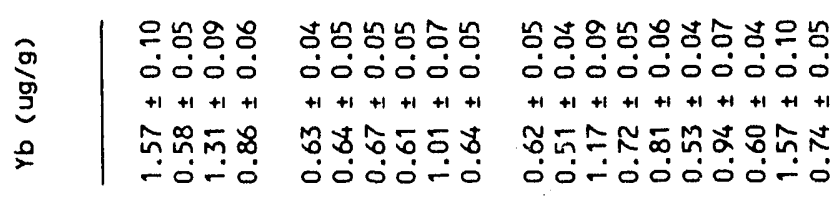

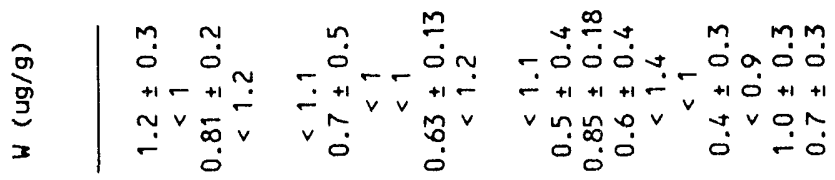

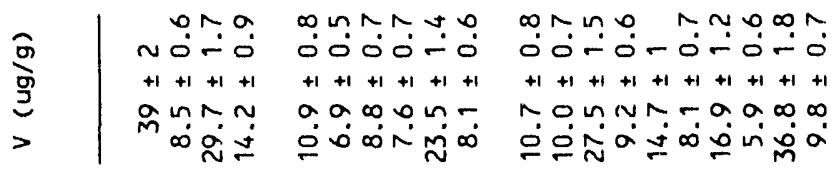

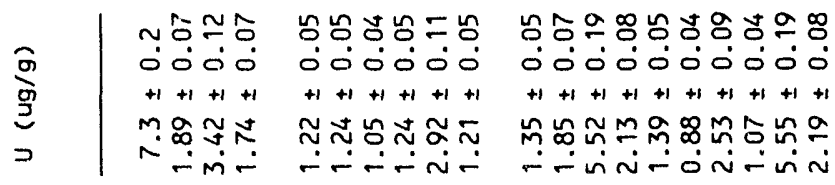

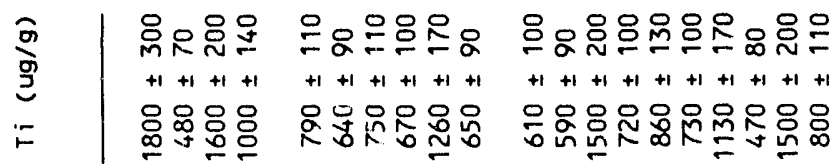

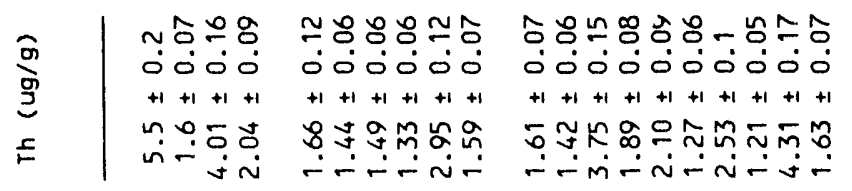

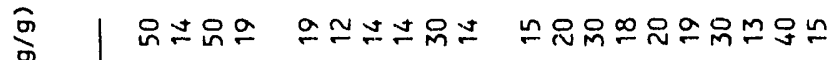
马) $+1+1+1+1+1+1+1+1+1+1+1+1+1+1+1+1+1+1+1+1$

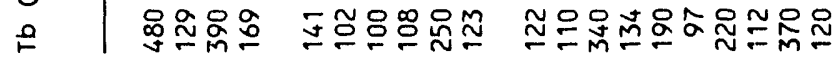

吉自|

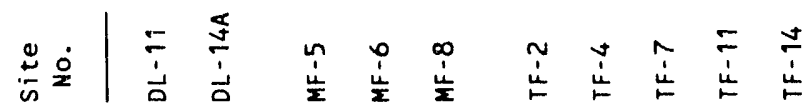




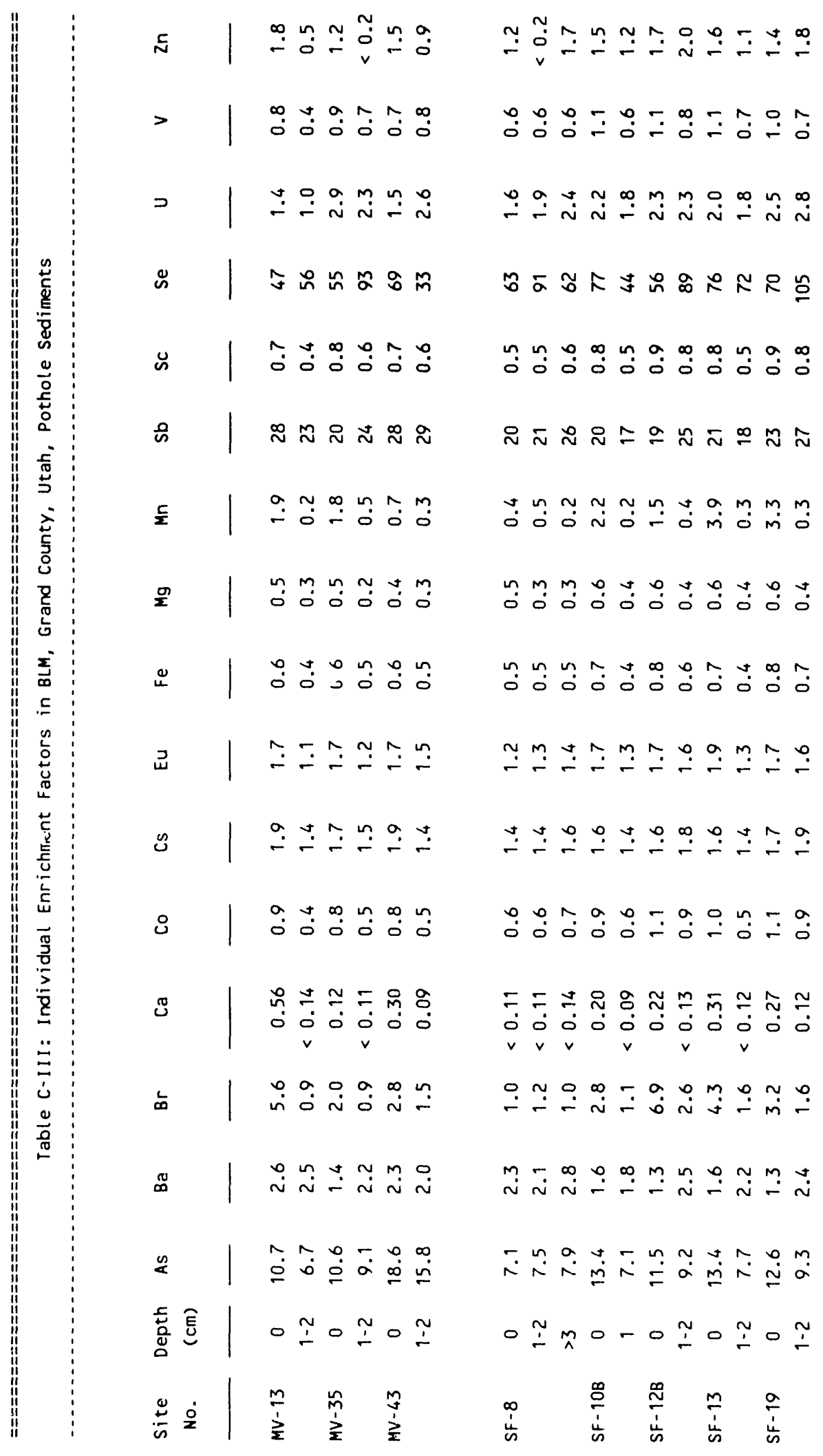




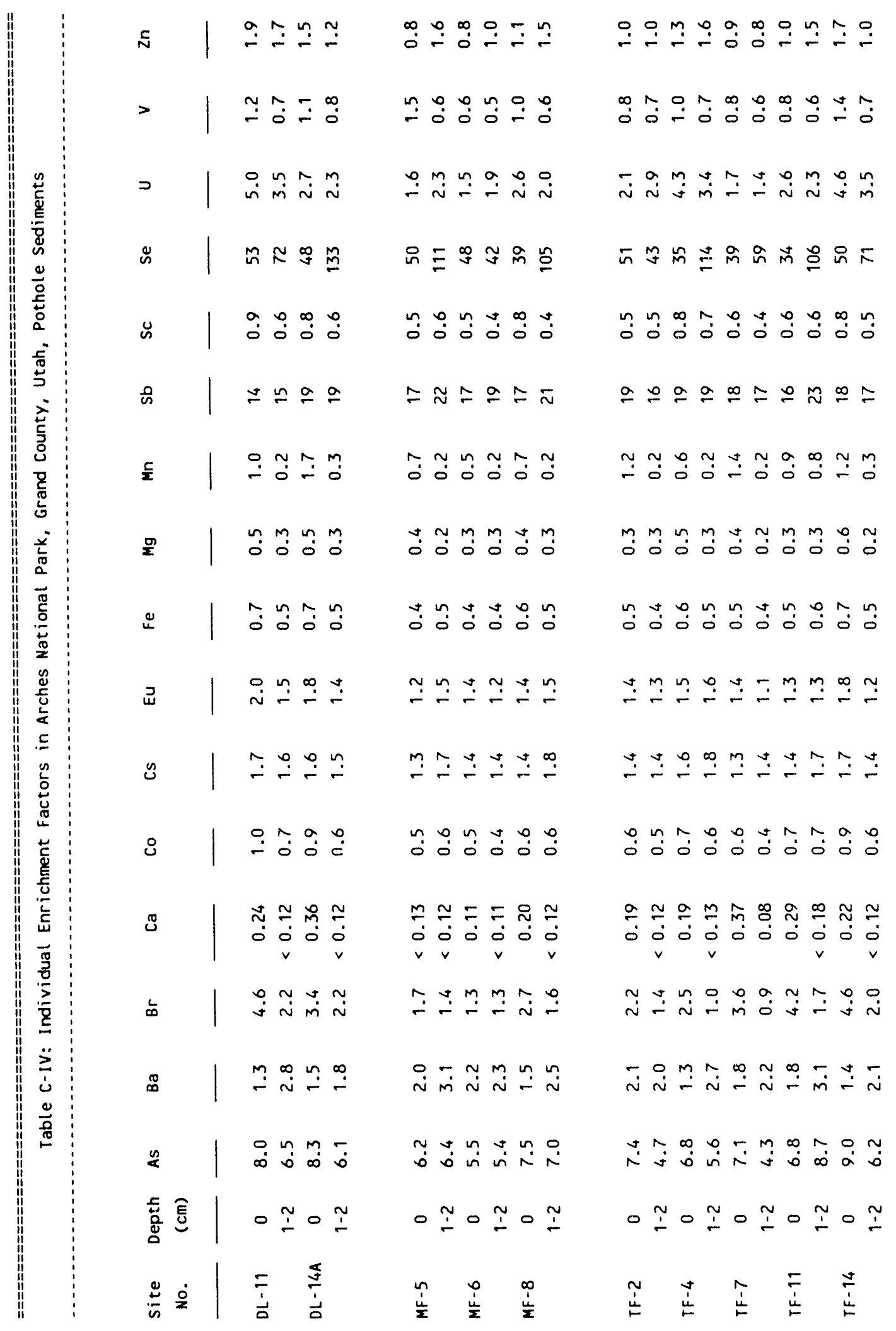


Appendix D: In Situ Temperature, pH, and Alkalinity Measurements in Potholes 


\begin{tabular}{|c|c|c|c|c|c|c|c|c|c|c|c|}
\hline $\begin{array}{c}\text { site } \\
\text { No. }\end{array}$ & Date & Time & Temp & $\mathbf{p H}$ & Alk & $\begin{array}{c}\text { site } \\
\text { No. }\end{array}$ & Date & Time & Temp & $\mathrm{pH}$ & Alk \\
\hline \multirow[t]{2}{*}{ SF - 2} & $10 / 05 / 89$ & 1100 & 19.5 & 8.20 & 72.7 & $M V-5$ & $7 / 31 / 89$ & 1010 & 26.0 & 8.20 & 25.6 \\
\hline & $10 / 16 / 89$ & 1030 & 17.5 & $? .61$ & 38.5 & & $8 / 15 / 89$ & 1500 & 32.8 & 8.33 & 21.4 \\
\hline \multirow[t]{6}{*}{ SF -8} & $8 / 21 / 88$ & $\cdots$ & 22.7 & 9.61 & 38.5 & & $10 / 16 / 89$ & 1355 & 23.0 & 8.78 & 29.9 \\
\hline & $9 / 25 / 88$ & $\cdots$ & 19.6 & 9.25 & 21.4 & $M V-11$ & $7 / 31 / 89$ & 1020 & 24.1 & 7.72 & 29.9 \\
\hline & $7 / 31 / 89$ & 0825 & 21.0 & 7.28 & 21.4 & & $8 / 15 / 89$ & 1450 & 31.4 & 7.62 & 25.6 \\
\hline & $8 / 15 / 89$ & 1150 & 24.6 & 6.91 & 17.1 & & $10 / 16 / 89$ & 1415 & 19.6 & 7.52 & 25.6 \\
\hline & $10 / 05 / 89$ & 1150 & 19.9 & 7.42 & 29.9 & $M V-13$ & $7 / 25 / 89$ & 1435 & 35.3 & 7.40 & 34.2 \\
\hline & $10 / 16 / 89$ & 1220 & 19.4 & 7.36 & 17.1 & & $7 / 31 / 89$ & 1035 & 27.6 & 6.55 & 25.6 \\
\hline \multirow[t]{2}{*}{$S F-9 A$} & $10 / 05 / 89$ & 1225 & 19.0 & 7.40 & 38.5 & & $9 / 13 / 89$ & 1055 & 22.8 & 7.80 & 17.0 \\
\hline & $10 / 16 / 89$ & 1210 & 18.6 & 7.38 & 21.4 & & $10 / 16 / 89$ & 1440 & 20.9 & 8.52 & 29.9 \\
\hline \multirow[t]{7}{*}{ SF-10B } & $8 / 21 / 88$ & $\cdots$ & 22.6 & 9.06 & 81.2 & $M V-14 A$ & $7 / 31 / 89$ & 1045 & 28.1 & 8.27 & 38.5 \\
\hline & $9 / 25 / 88$ & $\cdots$ & 14.4 & 8.89 & 55.6 & & $8 / 15 / 89$ & 1435 & 33.1 & 7.56 & 25.6 \\
\hline & $7 / 25 / 89$ & 1340 & 33.2 & 7.31 & 21.4 & & $10 / 05 / 89$ & 1455 & 19.7 & 9.01 & 38.5 \\
\hline & $7 / 31 / 89$ & 0855 & 21.0 & 7.87 & 21.4 & & $10 / 16 / 89$ & 1515 & 21.5 & 8.87 & 21.4 \\
\hline & $8 / 15 / 89$ & 1110 & 24.1 & 6.83 & 21.4 & $M V-14 B$ & $9 / 13 / 89$ & 1125 & 23.4 & 7.28 & 29.9 \\
\hline & $10 / 05 / 89$ & 1255 & 18.0 & 7.42 & 29.9 & & $10 / 16 / 89$ & 1500 & 19.2 & 7.76 & 17.1 \\
\hline & $10 / 16 / 89$ & 1105 & 16.5 & 7.33 & 21.4 & $M V-25$ & $7 / 31 / 89$ & 1100 & 28.1 & 7.71 & 12.0 \\
\hline \multirow[t]{7}{*}{$S F-12 B$} & $8 / 21 / 88$ & $\cdots$ & 22.5 & 7.97 & 77.0 & & $8 / 15 / 89$ & 1420 & 33.9 & 7.92 & 29.9 \\
\hline & $9 / 25 / 88$ & $\cdots$ & 14.3 & 9.16 & 55.6 & & $9 / 13 / 89$ & 1150 & 24.5 & 8.18 & 28.0 \\
\hline & $7 / 25 / 89$ & 1340 & 30.3 & 7.30 & 29.9 & & $10 / 16 / 89$ & 1550 & 15.0 & 8.67 & 11.0 \\
\hline & $7 / 31 / 89$ & 0910 & 22.6 & 7.84 & 25.6 & $M V-35$ & $8 / 15 / 89$ & 1400 & 32.0 & 6.88 & 21.4 \\
\hline & $8 / 15 / 89$ & 1115 & 24.5 & 6.98 & 21.4 & & $10 / 16 / 89$ & 1610 & 16.5 & 8.25 & 34.2 \\
\hline & $10 / 05 / 89$ & 1320 & 23.6 & 8.07 & 38.5 & & & & & & \\
\hline & $10 / 16 / 89$ & 1130 & 18.3 & 7.34 & 21.4 & & & & & & \\
\hline \multirow[t]{7}{*}{$S F-13$} & $8 / 21 / 88$ & $\cdots$ & 22.4 & 8.99 & 72.7 & & & & & & \\
\hline & $9 / 25 / 88$ & $\cdots$ & 14.4 & 9.40 & 51.3 & & & & & & \\
\hline & $7 / 25 / 89$ & 1350 & 34.6 & 7.47 & 47.0 & & & & & & \\
\hline & $7 / 31 / 89$ & 0915 & 21.8 & 7.89 & 25.6 & & & & & & \\
\hline & $8 / 15 / 89$ & 1120 & 24.2 & 7.10 & 21.4 & & & & & & \\
\hline & $10.05 / 89$ & 1340 & 25.3 & 8.46 & 34.2 & & & & & & \\
\hline & $10 / 16 / 89$ & 1150 & 19.1 & 7.30 & 17.1 & & & & & & \\
\hline \multirow[t]{2}{*}{$S F-18$} & $10 / 05 / 89$ & 1410 & 24.4 & 8.52 & 51.3 & & & & & & \\
\hline & $10 / 16 / 89$ & 1255 & 23.9 & 7.69 & 25.6 & & & & & & \\
\hline \multirow[t]{5}{*}{ SF- 19} & $8 / 21 / 88$ & $\cdots$ & 22.5 & 8.90 & 72.7 & & & & & & \\
\hline & $9.25 / 88$ & $\cdots$ & 15.8 & 9.22 & 38.5 & & & & & & \\
\hline & $7 / 25 / 89$ & 0930 & 22.5 & 7.47 & 29.9 & & & & & & \\
\hline & $8 / 15 / 89$ & 1130 & 25.0 & 7.31 & 21.4 & & & & & & \\
\hline & $10 / 16 / 89$ & 1315 & 22.5 & 7.57 & 21.4 & & & & & & \\
\hline
\end{tabular}

\footnotetext{
Note: Temperature in degrees $\mathrm{C}$, $\mathrm{pH}$ in units, and $\mathrm{Alkal}$, inity in $\mathrm{mg} / \mathrm{L} \mathrm{CaCO}_{3}$.
} 


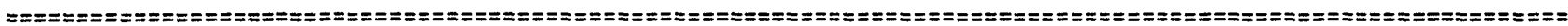

Table D-II: In Situ Measurements of Temperature, PH, an: Alkalinity, Arches National Park Potholes, Grand County, Utah

\begin{tabular}{|c|c|c|c|c|c|c|c|c|c|c|c|}
\hline $\begin{array}{l}\text { site } \\
\text { No. }\end{array}$ & Date & Time & Temp & PH & Alk & $\begin{array}{c}\text { Site } \\
\text { No. }\end{array}$ & Date & Time & Temp & PH & Alk \\
\hline \multirow[t]{7}{*}{ If -2} & $8 / 21 / 88$ & ... & 29.5 & 7.35 & 22.4 & $D L-11$ & $8 / 21 / 88$ & $\cdots$ & 26.6 & 7.46 & 17.1 \\
\hline & $9 / 22 / 88$ & $\ldots$ & 26.8 & 8.85 & 29.9 & & $9 / 22 / 88$ & $\cdots$ & 26.5 & 9.50 & 29.9 \\
\hline & $6 / 12 / 89$ & 1715 & 24.5 & 7.35 & 21.4 & & $7 / 30 / 89$ & 1050 & 28.8 & 6.49 & 25.6 \\
\hline & $7 / 24 / 89$ & 1020 & 29.0 & 6.63 & 25.6 & & $8 / 16 / 89$ & 1715 & 26.0 & 6.67 & 29.9 \\
\hline & $7 / 30 / 89$ & 1350 & 34.0 & 9.62 & 25.6 & & & & & & \\
\hline & $8 / 16 / 89$ & 1120 & 28.1 & 7.47 & 29.9 & $D L-14 A$ & $8 / 21 / 88$ & $\cdots$ & 28.7 & 7.33 & 22.4 \\
\hline & & & & & & & 9/22/88 & $\cdots$ & 23.8 & 8.28 & 34.2 \\
\hline \multirow[t]{4}{*}{ TF -4} & $8 / 21 / 88$ & $\cdots$ & 28.8 & 7.19 & 17.1 & & $7 / 30 / 89$ & 1115 & 29.1 & 7.09 & 25.6 \\
\hline & $9 / 22 / 88$ & $\cdots$ & 24.0 & 8.28 & 29.9 & & $8 / 16 / 89$ & 1105 & 26.8 & 7.13 & 38.5 \\
\hline & $7 / 30 / 89$ & 1335 & 35.9 & 8.64 & 21.4 & & & & & & \\
\hline & & & & & & MF -5 & $8 / 21 / 88$ & $\cdots$ & 30.5 & 8.83 & 25.7 \\
\hline \multirow[t]{7}{*}{ TF -7} & $8 / 21 / 88$ & $\ldots$ & 29.2 & 8.80 & 29.9 & & $7 / 30 / 89$ & 1205 & 32.5 & 7.90 & 17.1 \\
\hline & $9 / 22 / 88$ & $\ldots$ & 25.3 & 9.07 & 29.8 & & $8 / 16 / 89$ & 1225 & 29.4 & 7.77 & 25.6 \\
\hline & $6 / 12 / 89$ & 1735 & 26.8 & 8.80 & 47.0 & & & & & & \\
\hline & $7 / 24 / 89$ & 1035 & 30.4 & 6.79 & 38.5 & $M F-6$ & $8 / 21 / 88$ & $\cdots$ & 31.2 & 8.97 & 21.4 \\
\hline & $7 / 30 / 89$ & 1320 & 35.6 & 9.04 & 21.4 & & $9 / 22 / 88$ & $\cdots$ & 28.3 & 9.47 & 29.9 \\
\hline & $8 / 16 / 89$ & 1135 & 28.1 & 6.93 & 29.9 & & $6 / 12 / 89$ & 1850 & 23.1 & 8.3 & 25.6 \\
\hline & & & & & & & $7 / 30 / 89$ & 1215 & 34.0 & 6.72 & 29.9 \\
\hline \multirow[t]{6}{*}{$T F-11$} & $8 / 21 / 88$ & $\cdots$ & 29.2 & 7.69 & 21.4 & & $8 / 16 / 89$ & 1220 & 32.3 & 9.14 & 21.4 \\
\hline & $9 / 22 / 88$ & $\cdots$ & 26.0 & 9.55 & 34.2 & & & & & & \\
\hline & $6 / 12 / 89$ & 1820 & 21.8 & 8.55 & 42.8 & $M F-8$ & $8 / 21 / 88$ & $\cdots$ & 30.0 & 8.14 & 21.4 \\
\hline & $7 / 30 / 89$ & 1300 & 33.9 & 9.26 & 25.6 & & $9 / 22 / 88$ & $\cdots$ & 27.7 & 9.24 & 29.9 \\
\hline & $8 / 16 / 89$ & 1150 & 29.0 & 7.57 & 29.9 & & $6 / 12 / 89$ & 1915 & 22.1 & 7.71 & 29.4 \\
\hline & & & & & & & $7 / 24 / 89$ & 1110 & 32.0 & 6.89 & 21.4 \\
\hline \multirow[t]{4}{*}{$T F-14$} & $8 / 21 / 88$ & $\cdots$ & 30.8 & 7.68 & 21.4 & & $7 / 30 / 89$ & 1235 & 33.3 & 7.54 & 34.2 \\
\hline & $9 / 22 / 88$ & $\cdots$ & 28.3 & 9.51 & 21.4 & & $8 / 16 / 89$ & 1230 & 25.7 & 8.14 & 21.4 \\
\hline & $8 / 16 / 89$ & 1245 & 32.3 & 9.20 & 21.4 & & & & & & \\
\hline & & & & & & $M F-9$ & $9 / 22 / 88$ & $\cdots$ & 25.9 & 8.92 & 47.0 \\
\hline
\end{tabular}

Note: Temperature in degrees $\mathrm{C}, \mathrm{PH}$ in units, and Alkalinity in $\mathrm{mg} / \mathrm{L} \mathrm{CaCO}_{3}$. 


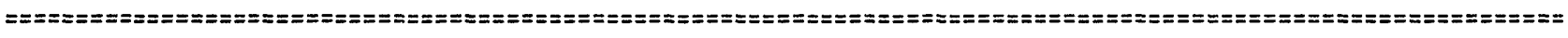
Table D-III: In Situ Measurements of Temperature, PH, and Alkalinity in LAME and JOTR

Lake Meade National Recreation Area

\begin{tabular}{|c|c|c|c|c|c|}
\hline $\begin{array}{c}\text { Site } \\
\text { No. }\end{array}$ & Date & Time & Temp & $\mathrm{pH}$ & $A \mid k$ \\
\hline CAT - 1 & $5 / 29 / 90$ & 1400 & 26.5 & 7.64 & 42.7 \\
\hline CAT -2 & $5 / 29 / 90$ & 1040 & 22.4 & 8.41 & 17.1 \\
\hline CAT -3 & $5 / 29 / 90$ & 1055 & 23.3 & 8.97 & 12.8 \\
\hline CAT -4 & $5 / 29 / 90$ & 1145 & 26.1 & 8.98 & 12.8 \\
\hline CAT -5 & $5 / 29 / 90$ & 1310 & 27.0 & 9.46 & 17.1 \\
\hline LAKE- 1 & $5 / 29 / 90$ & 1540 & 29.9 & 9.84 & 34.2 \\
\hline GRAPE - 1 & $5 / 31 / 90$ & 1240 & 20.5 & 10.63 & 29.9 \\
\hline GRAPE - 2 & $5 / 31 / 90$ & 1300 & 19.4 & 9.20 & 34.2 \\
\hline
\end{tabular}

Joshua Tree National Monument

\begin{tabular}{|c|c|c|c|c|c|}
\hline $\begin{array}{l}\text { Site } \\
\text { No. }\end{array}$ & Date & Time & Temp & $\mathrm{pH}$ & Alk \\
\hline \multirow[t]{2}{*}{ HO- 1} & $6 / 11 / 90$ & 1430 & 30.4 & 9.99 & 42.8 \\
\hline & $7 / 11 / 90$ & 1405 & 35.4 & 9.56 & 12.8 \\
\hline \multirow[t]{2}{*}{ HO-2 } & $6 / 11 / 90$ & 1530 & 25.4 & 8.81 & $<1$ \\
\hline & $7 / 11 / 90$ & 1505 & 33.1 & 9.00 & 4.3 \\
\hline \multirow[t]{2}{*}{ HO-3 } & $6 / 11 / 90$ & 1600 & 24.3 & 8.83 & $<1$ \\
\hline & $7 / 11 / 90$ & 1530 & 32.4 & 9.20 & 8.6 \\
\hline \multirow[t]{2}{*}{$B O-2$} & $2 / 21 / 90$ & 1410 & 8.8 & 9.14 & 34.2 \\
\hline & $7 / 18 / 90$ & 1530 & 32.2 & 10.05 & 51.3 \\
\hline BO-3 & $2 / 21 / 90$ & 1450 & 9.6 & 9.09 & $\cdots$ \\
\hline BO -4 & $7 / 18 / 90$ & 1340 & 32.3 & 10.01 & 17.1 \\
\hline$B O-5$ & $7 / 18 / 90$ & 1405 & 33.4 & 10.21 & 38.5 \\
\hline
\end{tabular}

Note: Temperature in degrees $\mathrm{C}, \mathrm{PH}$ in units, and $\mathrm{Alkal}$ inity in $\mathrm{mg} / \mathrm{L} \mathrm{CaCO}_{3}$. 

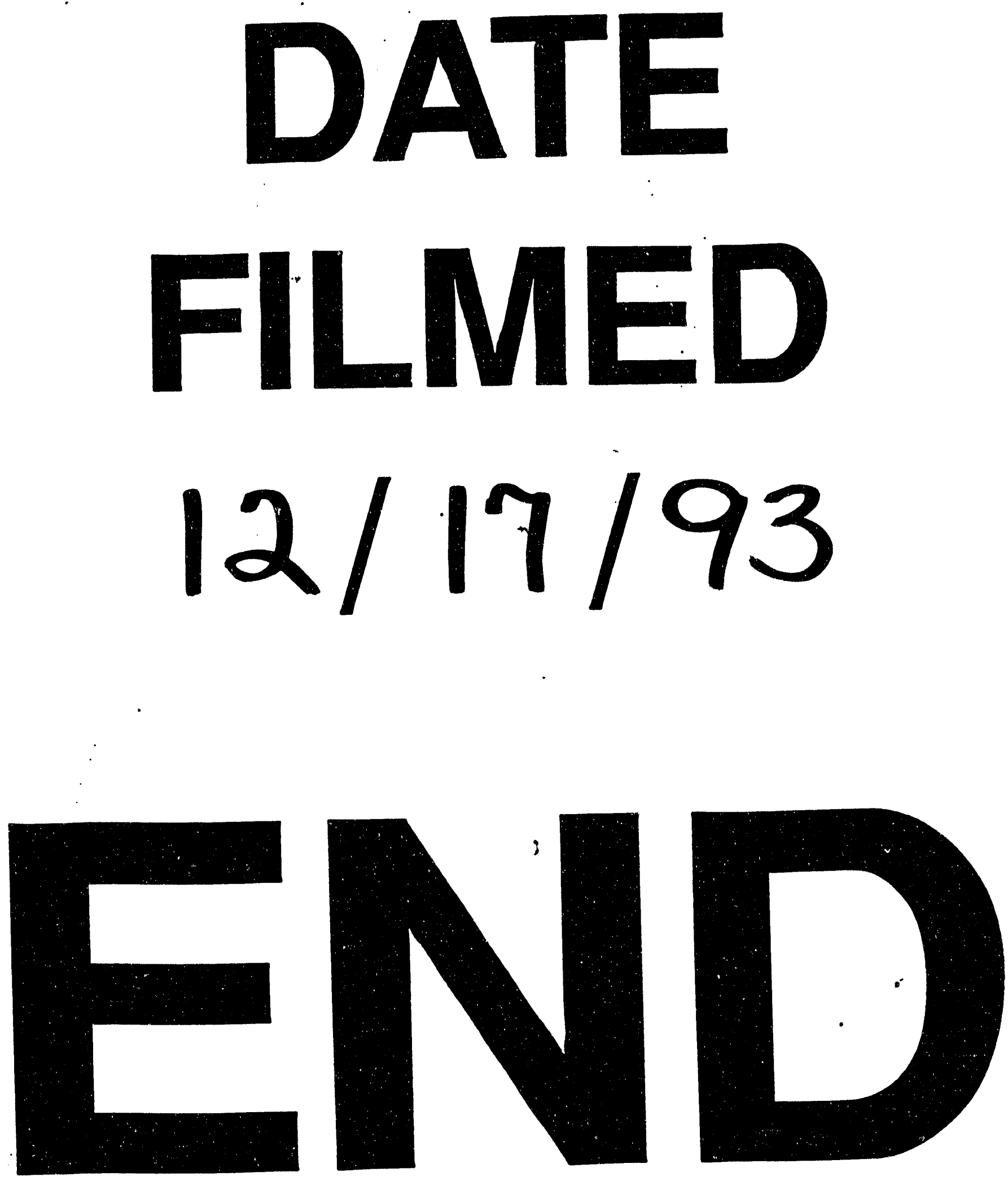
